\title{
Electro-Optic Modulation using a Polymeric Microring Resonator with Highly Photostable Chromophores
}


This research has been supported by the Dutch Technology Foundation STW, applied science division of NWO, TOE 6067.

This Research was carried out at the Integrated Optical Microsystems (IOMS) group, Faculty of Electrical Engineering, Mathematics and Computer Science, MESA ${ }^{+}$institute for Nanotechnology, University of Twente P. O. Box 217, 7500AE Enschede, The Netherlands.

Copyright 2008 by Muralidharan Balakrishnan, Enschede, The Netherlands.

ISBN: 978-90-365-2730-9 


\title{
Electro-Optic Modulation using a Polymeric Microring Resonator with Highly Photostable Chromophores
}

\author{
DISSERTATION
}

to obtain

the degree of doctor at the University of Twente, on the authority of the rector magnificus, prof.dr. W.H.M. Zijm,

on account of the decision of the graduation committee, to be publicly defended

on Friday the $19^{\text {th }}$ of September 2008 at 13.15

by

Muralidharan Balakrishnan

born on the $16^{\text {th }}$ of April 1979

in Mayiladuthurai, India. 
This dissertation is approved by: promotor: Prof. Dr. A. Driessen 
To my parents.... 

2 Nonlinear optical polymers for electro-optic modulation

2.1 Introduction

2.2 Origin of nonlinearity in polymers $\quad 8$

$\begin{array}{ll}\text { 2.2.1 Second order nonlinearity } & 8\end{array}$

$\begin{array}{ll}\text { 2.2.2 Linear electro-optic effect (pockels effect) } & 11\end{array}$

$\begin{array}{ll}2.3 \text { Different classes of NLO polymers } & 13\end{array}$

2.4 Chromophore orientation 14

2.5 Material requirements for an electro-optic modulator 16

$\begin{array}{ll}2.6 \text { processing and fabrication } & 19\end{array}$

2.6.1 Spin coating 19

2.6.2 Fabrication techniques $\quad 20$

$\begin{array}{ll}2.7 \text { Device configurations } & 20\end{array}$

2.8 References $\quad 21$

3 Characterization and optimizing the properties of the TCVDPA chromophore for application in EO modulators 23

3.1 Introduction 24

3.2 Properties of the TCVDPA chromophore $\quad 25$

3.2.1 Refractive index measurements 25

3.2.2 Photodefinition by UV-thermal crosslinking 26

3.2.3 Functionalization of TCVDPA with bulky groups $\quad 27$

3.2.4 Slab waveguide loss measurements $\quad 28$

3.2.5 Thermal properties of TCVDPA $\quad 30$

3.2.6 Photobleaching experiments of TCVDPA 31

3.3 Poling 33

3.4 Measurement of $\mathrm{r}_{33}$ with the aid of the Teng-Man setup 36

$\begin{array}{ll}\text { 3.4.1 Sample preparation } & 41\end{array}$

3.5 In-situ poling with the Teng-Man setup $\quad 42$

$\begin{array}{ll}\text { 3.5.1 Monitoring birefringence } & 42\end{array}$

3.5.2 Monitoring the EO modulation amplitude 44

3.6 Enhancement of poling efficiency 45

3.7 Effect of chromophore concentration on $\mathrm{T}_{\mathrm{g}} \quad 48$

3.8 Relaxation measurements $\quad 49$

3.9 Conclusions $\quad 51$

3.10 References $\quad 52$

4 Design and fabrication of laterally coupled PMMA-DR1

microring resonators $\quad 55$

4.1 Introduction 56

4.1.1 Working principle of an optical microring resonator 56

$\begin{array}{ll}\text { 4.1.2 Microring losses } & 58\end{array}$

4.1.3 Bandwidth limitations $\quad 59$

4.2 Microring resonator design $\quad 59$

$\begin{array}{ll}4.3 \text { Different fabrication methods } & 64\end{array}$ 
4.3.1 Photodefinition $\quad 64$

4.3.2 Embossing $\quad 66$

$\begin{array}{ll}\text { 4.3.3 Reactive ion etching in VSC } & 67\end{array}$

$\begin{array}{ll}\text { 4.3.4 Inverted ridges in } \mathrm{SiO}_{2} \text { (Hybrid approach) } & 69\end{array}$

$\begin{array}{ll}\text { 4.3.5 Measurements of the microrings made by hybrid approach } & 70\end{array}$

$\begin{array}{ll}4.4 \text { Racetrack resonator } & 73\end{array}$

$\begin{array}{ll}\text { 4.4.1 Racetrack design } & 73\end{array}$

$\begin{array}{ll}\text { 4.4.2 Racetrack fabrication by hybrid approach } & 75\end{array}$

$\begin{array}{ll}\text { 4.4.3 Poling of racetrack devices } & 77\end{array}$

$\begin{array}{ll}4.5 \text { Conclusions } & 78\end{array}$

$\begin{array}{ll}4.6 \text { References } & 78\end{array}$

5 MR-based modulator made by direct photodefinition of an

electro-optic polymer $\quad 81$

$\begin{array}{ll}5.1 \text { Introduction } & 82\end{array}$

5.1.1 Requirements of the resist core material $\quad 83$

$\begin{array}{ll}5.2 \mathrm{SU} 8 \text { negative resist as core material } & 83\end{array}$

$\begin{array}{ll}\text { 5.2.1 SU8 processing } & 83\end{array}$

5.2.2 Chemical characteristics of SU8 84

5.2.3 Commercially available SU8 formulations 86

$\begin{array}{ll}\text { 5.2.4 Lithography with SU8 } & 87\end{array}$

5.3 SU8-TCVDPA EO polymers $\quad 88$

$\begin{array}{ll}\text { 5.3.1 Poling behavior of SU8-TCVDPA EO polymer } & 89\end{array}$

5.4 Fabrication of MRs by photodefinition $\quad 93$

5.4.1 Optimization of the lithographic process 93

5.4.2 Device fabrication schemes 95

5.4.2.1 Fabrication by simultaneous poling and crosslinking (scheme 1) 95

5.4.2.2 Fabrication by initial partial crosslinking (scheme 2) 96

5.4.2.3 Alternative fabrication method (scheme 3) 97

$\begin{array}{ll}5.5 \text { Experimental measurements } & 97\end{array}$

$\begin{array}{ll}\text { 5.5.1 Measurement of the ring response } & 97\end{array}$

5.5.2 Analysis of the ring losses 99

$\begin{array}{ll}\text { 5.5.3 Measurement of EO modulation } & 100\end{array}$

$\begin{array}{ll}\text { 5.5.4 Epoxy-gold reactions } & 104\end{array}$

$\begin{array}{ll}5.6 \text { Conclusions } & 104\end{array}$

$\begin{array}{ll}5.7 \text { References } & 105\end{array}$

6 NLO Polycarbonates 107

6.1 Introduction 108

6.2 Functionalization of PC with TCVDPA 109

$\begin{array}{ll}\text { 6.2.1 Relaxation behavior } & 113\end{array}$

6.3 Functionalization of PC with high $\mu \beta$ chromophores $\quad 118$

$\begin{array}{lr}6.4 \text { Conclusions } & 120\end{array}$

$\begin{array}{ll}6.5 \text { References } & 120\end{array}$

$\begin{array}{ll}7 \text { Summary and conclusions } & 123\end{array}$

ii 
Appendix 1

List of publications

Acknowledgments 


\section{Introduction}

In optical communication systems, electro-optic (EO) modulators are used to encode electrical input data signals onto fiber optic transmission lines. The dominant EO material in the presently applied technology is Lithium Niobate. Commercially available Lithium Niobate modulators are limited in their bandwidth to $40 \mathrm{GHz}$ due to the velocity mismatch between the electrical drive signal and the optical transmission signal [1]. For the next generation optical communication systems with data rates $>40 \mathrm{~Gb} / \mathrm{s}$ a better performing material is required. EO polymers have been considered as an alternative already two decades ago. However, their EO activity appeared to be disappointingly low, while the materials were also prone to photochemical degradation. This has prevented the use of EO polymers in practical applications up to now. A recent finding to prevent antiparallel clustering of the EO molecules by attaching bulky side groups, has resulted in EO polymer modulators with record-setting bandwidths in excess of $100 \mathrm{GHz}(165 \mathrm{GHz})$ and low drive voltages (sub-1V) [2]. As the currently available EO polymers are prone to photodegradation because of oxygen attack, hermetic packaging is required for long term device application. In the current work the main focus is directed on the development of a highly photostable EO polymer by making use of a chromophore (TCVDPAtricyanovinylidenediphenylaminobenzene) which is stated to have the highest known photo-chemical stability in the literature [3,4]. As the EO effect in this chromphore is relatively low, efforts are made to modify its shape in order to increase its poling efficiency.

EO polymers also allow for the realization of an efficient EO tunable microring resonator. We will focus on this component, because it yields a new opto-electronic compact device for efficient EO modulation [5-8]. This functionality is highly desired in dense wavelength multiplexed (DWDM) optical communication systems. With its small dimensions it allows for complex integrated optic structures with thousands of elements within a square centimeter of chip area. In combination with the low-cost polymer technology, it provides the technological and economical basis for various high performance components for applications in optical communication networks.

The outline of the thesis is as follows. Chapter 2 gives the basics of nonlinear optics of EO polymers. Several classes of EO polymers are discussed and the material requirements for EO modulators and fabrication process are explained.

Chapter 3 presents in detail the EO coefficient $\left(\mathrm{r}_{33}\right)$ measurement set-up. Also the physical and chemical properties of the TCVPDA chromophore and its modifications are given. Results of the loss measurement and $r_{33}$ of TCVDPA series of chromophore are presented. The possibility of direct photodefinition of a host polymer loaded with the TCVDPA chromophore is highlighted. Photobleaching tests were carried out to compare the photostability of the TCVDPA chromophore with traditional chromophores. Enhancement of the poling efficiency by modification of TCVDPA with bulky side groups is shown.

Chapter 4 deals with the design and fabrication of laterally coupled microring resonators. Different fabrication techniques for the realization of the devices are explained and the measured ring responses are presented. 
Chapter 5 presents the novel method of direct photodefinition of a chromophore (TCVDPA) containing host material. The main focus is given to the optimization of the poling of the chromophore and crosslinking of the host. Fabrication recipes for realizing microring resonators by this method is given. EO modulation of poled microring resonators structured by photodefinition is demonstrated.

Chapter 6 presents a new class of polymers made by attaching the TCVDPA chromophore to the polymer. The effect of different ways of attachment on the poling efficiency is studied. This chapter also presents the relaxation behavior of the different kinds of PCTCVDPA polymers.

The results of all the chapters are summarized in chapter 7.

\section{References}

[1] Y. Enami, C. T. Derose, D. Mathine, C. Loychik, C. Greenlee, R. A. Norwood, T. D. Kim, J. Luo, Y. Tian, A. K. Y. Jen, N. Peyghambarian, Nature Photonics, 1, p180, 2007.

[2] B. Bortnik, Y. C. Hung, H. Tazawa, B. J. Seo, J. Luo, A. K. Y. Jen, W. H. Steier, H. R. Fetterman, J. of Selected Topics in Quantum Electronics, 10, p1, 2007.

[3] A. Galvan-Gonzalez, M. Canva, G. I. Stegeman, R. Twieg, T. C. Kowalczyk, H. S. Lackritz, Opt. Lett., 24, p1741, 1999.

[4] A. Galvan-Gonzalez, M. Canva, G. I. Stegeman, R. Twieg, K. P. Chan, T. C. Kowalczyk, X. Q. Zhang, H. S. Lackritz, S. Marder, S. Thayumanavan, Opt. Lett., 25, p332, 2000.

[5] H. Tazawa, Y. H. Kuo, I. Dunayevskiy, J. Juo, A. K. Y. Jen, H. R. Fetterman, W. H. Steier, J. of Lightwave Technology, 24, p3514, 2006.

[6] E. J. Klein, D. H. Geuzebroek, H. Kelderman, G. Sengo, N. Baker, A. Driessen, IEEE Photonics Technology Letters, 17, p2358, 2005.

[7] B. E. Little, S. T. Chu, P. P. Absil, J. V. Hryniewicz, F. G. Johnson, F. Seiferth, D. Gill, V. Van, O. King, M. Trakalo, , IEEE Photonics Technology Letters, 16, p2263, 2004.

[8] S. Xiao, M. H. Khan, H. Shen, M. Qi, , J. of Lightwave Technology, 26, p228, 2008. 
2 Nonlinear optical polymers for electro-optic modulation

This chapter presents the theoretical background of electro-optic polymers for applications in electro-optic modulators. The physical origin of the nonlinear effect is discussed. Several configurations of nonlinear optical polymers are described and a discussion is given for their merits. Finally some important requirements for electro-optic polymer are presented. 


\subsection{Introduction}

Considerable interest has been shown for the development and application of organic nonlinear optical materials for EO active devices. [1-3]. Polymeric materials appear to have several advantages in comparison with inorganic materials for application in electrooptic modulators. This interest stems from the potential of these materials for low cost products which cannot be achieved with the expensive inorganic crystal growing technology. The key advantage is the nonlinearity being purely electronic in origin corresponding to a response time of few femtoseconds. One more important advantage of polymers is their inherently low dielectric constant at RF fields of about 3, which allows the electrical driving field and waves at optical frequencies to co-propagate for long distances without de-phasing. Inorganic materials have high dielectric constants which set their limit for the RF bandwidth to about $40 \mathrm{GHz}$, though through some special electrode designs bandwidths beyond $100 \mathrm{GHz}$ have been demonstrated. Polymers can also be deposited on different kinds of substrates including semiconductor. This enables a significant step forward in optoelectronic integration [4]. The processing steps of polymers and their temperature stability are compatible with semiconductor electronics [4]. Table 1 gives a comparison between the properties of organic and inorganic materials. It can be seen that polymers have clear advantages over the inorganic materials in terms of bandwidth and operational voltage (High bandwidth requires small device lengths and low voltage operation requires longer device lengths. Therefore the bandwidth-length product should be high and the voltage-length product should be low).

\subsection{Origin of nonlinearity in polymers}

Optical nonlinearity in polymers is due to the presence of active molecules called chromophores. The optical response of a chromophore depends on the mobility of the electrons. The high nonlinearity of organic molecules is due to the existence of so called $\pi$-bonds connecting, e.g., carbon atoms with each others. It is beyond the scope of this thesis to give a detailed review of bonding in molecules. However, a short introduction is adequate in order to demonstrate what parameters are crucial in optimizing the nonlinear properties of organic molecules. Molecules having extended overlapping $\pi$-bonds contain delocalized $\pi$-electrons that are shared by the whole system i.e. they are not localized in a particular bond but are free to move. Such molecules that contain $\pi$-bonds are called conjugated.

The mobility of bonding electrons increases with the order of the bonding (double or triple) and especially in aromatic rings. A chromophore essentially is a $\pi$-electron system as shown in Fig. 2.1. The polarizability of the chemical bond increases from 1 to 4 in Fig. 2.1 and is large only in the direction of the C-C bond. In the aromatic ring structure the polarizability is maximum in the ring plane and minimum in the direction perpendicular to it. 
Table 2.1: Comparison between organic and inorganic material properties.

\begin{tabular}{|c|c|c|c|}
\hline Property & Gallium Arsenide & Lithium Niobate & Polymers \\
\hline $\begin{array}{c}\text { EO coefficient } \\
(\mathrm{pm} / \mathrm{V} \text { at } 1.3 \mu \mathrm{m})\end{array}$ & 1.5 & 30 & $10-50$ \\
\hline Dielectric constant & 10 & 28 & $2.5-3$ \\
\hline Refractive index & 3.5 & 10 & $1.5-1.6$ \\
\hline $\begin{array}{c}\text { Bandwidth-length } \\
\text { product, GHz.cm }\end{array}$ & $>100$ & 5 & $>100$ \\
\hline $\begin{array}{c}\text { Voltage length } \\
\text { product V.cm }\end{array}$ & $1-5$ & 0.2 & $1-2$ \\
\hline $\begin{array}{c}\text { Optical loss } \\
(\mathrm{dB} / \mathrm{cm} \text { at } 1.3 \mu \mathrm{m})\end{array}$ & 2 & 90 & $0.2-1$ \\
\hline $\begin{array}{c}\text { Thermal stability } \\
{ }^{\circ} \mathrm{C}\end{array}$ & 80 & & $80-125$ \\
\hline
\end{tabular}

1

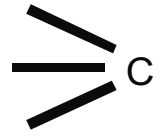

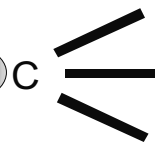

C
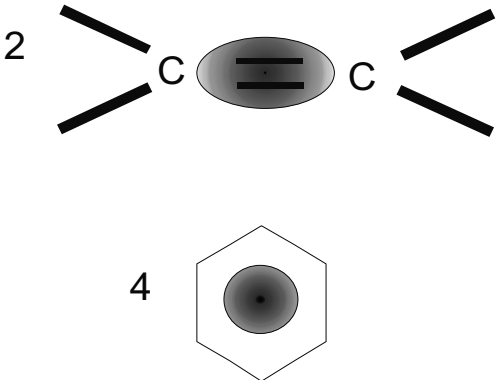

Figure 2.1: Increasing bond polarizability in $C-C$ bonds by successive introduction of increasingly delocalized $\pi$-electron systems. single bond (1), double bond (2), triple bond (3) and benzene ring (aromatic ring) (4).

The molecules in Fig. 2.1 as such remain centrosymmetric. The molecule will exhibit second order nonlinearity only if the centrosymmetry is broken. This can be achieved in a conjugated molecule by connecting each end of the conjugated path with groups that have different electron affinity. Symmetry is broken by the deformation of the $\pi$-electron distribution by attaching a donor like group at one end and an acceptor like group at the other end as shown schematically in Fig. 2.2 and the molecule has a dipole moment in its ground state. 


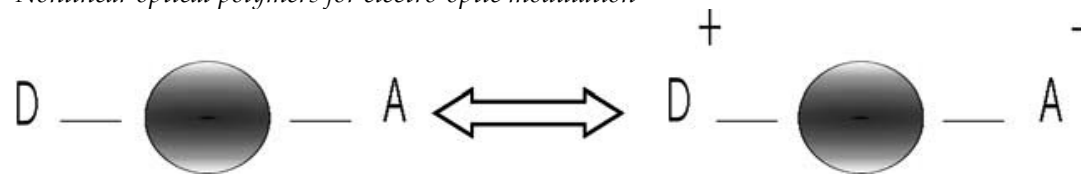

Figure 2.2: Delocalized $\pi$-electron system combined with donor and acceptor groups to form a charge transfer complex.

In Fig 2.2 the chromophore on the left hand side is in the ground state and that on the right hand side is in the charge transferred excited state. The molecule has a different dipole moment in the ground state and in the excited state. The response of the molecule to an electric field or its polarizability depends strongly on the direction of the applied field with respect to the molecule. Charge flow is favored towards the acceptor while hindered towards the donor. This asymmetric polarization provides strong second order nonlinear optical properties. Both the dipole moment and charge transfer affect the $\beta$ of the molecule. The first order molecular hyperpolarizability can be expressed as

$$
\beta=\frac{\left(\mu_{e}-\mu_{g}\right)\left(\mu_{g e}\right)^{2}}{(\Delta E)^{2}}
$$

Where, $\mu_{e}$ is the dipole moment of the molecule in the ground state, $\mu_{g}$ the dipole moment of the molecule in the excited state, $\mu_{g e}$ the transition dipole moment, and $\Delta E$ is the energy difference between ground and excited state. It can be seen from equation 1 that $\beta$ depends not on the ground state dipole moment of the molecule but on the difference between the dipole moments of the molecule in the ground and in the excited state.

The nonlinear optical activity of these molecules arises from their ability to change in a nonlinear way their polarizability under an external electric field. When an electric field $\mathrm{E}$ is applied to this molecule the induced polarization can be expressed in terms of the power series of the E field as,

$P_{i}=\alpha_{i j} E_{j}+\beta_{i j k} E_{j} E_{k}+\gamma_{i j k l} E_{j} E_{k} E_{l}+\ldots$

Where, $\alpha$ is the linear polarizability, $\beta$ and $\gamma$ are the first and second hyperpolarizabilities respectively. The indices ijk are the Cartesian components of the fields. When a polymer is loaded with these molecules the polarizability of the bulk material is in most cases zero as all the chromophores are randomly oriented. In this way, even with non-centrosymmetric molecules a macroscopically centrosymmetric material is created. To be electro-optic, a bulk material comprised of nonlinear molecules should also lack an inversion center. The method most widely used to impart polar order to non- 
crystalline systems is called poling. For this a high electric field in the order of $100 \mathrm{~V} / \mu \mathrm{m}$ is applied to the film heated up close to the glasstransition temperature $\left(T_{g}\right)$ so that the chromophores can align. The macroscopic polarization is then given by

$$
P_{I}=\chi_{I J}^{(1)} E_{J}+\chi_{I J K}^{{ }^{(2)}} E_{J} E_{K}+\chi_{I J K L}{ }^{(3)} E_{J} E_{K} E_{L}+\ldots
$$

The $\chi \mathrm{s}$ in the equation 3 are the macroscopic optical susceptibilities analogous to $\alpha$, $\beta, \gamma \ldots$ on the molecular scale. It is very difficult to relate the macroscopic susceptibility to the molecular hyperpolarizability. However, a simple description based on the oriented gas model has proven to be in many cases a good approximation for poled electro-optic polymers. Using this model the macroscopic second order nonlinear susceptibility in the orthogonal laboratory frame $\{X, Y, Z\}$, and the microscopic hyperpolarizability in the molecular frame $\{x, y, z\}$ (Fig. 2.3) can be related.

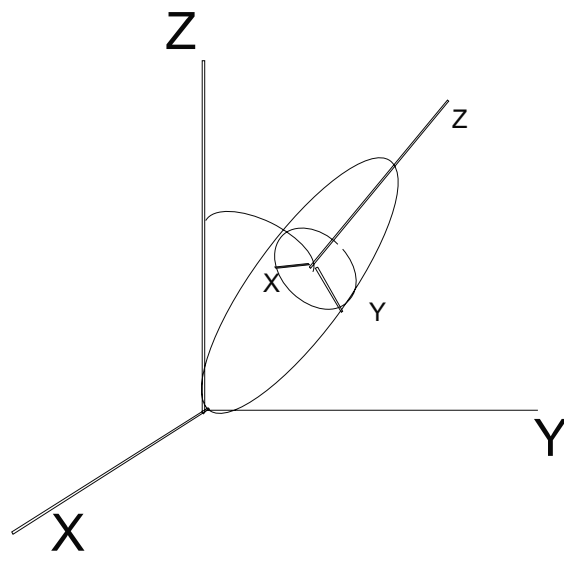

Figure 2.3: Laboratory $(X, Y, Z)$ and molecular $(x, y, z)$ co-ordinate axes.

Because of its simplicity, the oriented gas model relies on a large number of simplifications and approximations.

1. at the poling temperature the chromophores are assumed to rotate freely under the influence of the applied electrical field; any coupling or interaction with the surrounding matrix is ignored;

2. the chromophore has cylindrical symmetry and the only non vanishing hyperpolarizability tensor is $\beta_{z z z}$ where $\mathrm{z}$ is the charge transfer direction (symmetry axis) of the chromophore;

3. the permanent dipole moment $(\mu)$ of the molecule is oriented along the $\mathrm{z}$ axis of the molecule;

4. the chromophores are assumed to be independent and non-interacting. 
Under these conditions the bulk polarization response of the medium is given by the sum of the responses of the individual molecules as

$$
P_{I}(t)=\frac{1}{V}\left(\sum p_{i}(t)\right)_{I}
$$

Where, $V$ is the volume.

The second order nonlinear susceptibility tensor can be written as

$$
\chi_{z z z}^{(2)}=N \frac{\mu E_{p}}{5 k_{b} T} \beta_{z z z}
$$

$\chi_{z x x}^{(2)}=N \frac{\mu E_{p}}{15 k_{B} T} \beta_{z z z}$

Where $N$ is the chromophore number density, $E_{p}$ poling field strength, $k_{B}$ is Boltzmann's constant and $\mathrm{T}$ is the poling temperature.

With all the approximations made in this section we get that

$$
\chi^{(2)}{ }_{z x x}=\chi^{(2)}{ }_{z z z} / 3
$$

\subsubsection{Second order nonlinearity}

Equation 3 can be expanded to a form that more directly shows the existence of high frequency (for example frequency doubling: $f=2 \omega$ ) and low frequency (electro-optic: $f=$ 0) phenomena.

$$
\begin{aligned}
& P=\chi^{(1)} E_{0} \cos (\omega t-k z)+(1 / 2) \chi^{(2)} E_{0}{ }^{2}[1+\cos (2 \omega t-2 k z)]+\chi^{(3)} E_{0}{ }^{3}[(3 / 4) \\
& \cos (\omega t-k z)+(1 / 4) \cos (3 \omega t-3 k z)]
\end{aligned}
$$

The low frequency term involves frequencies in the range from $\mathrm{kHz}$ to $\mathrm{GHz}$. For example, radio, microwave and millimeter waves are low frequencies compared with optical frequencies (hundreds of $\mathrm{THz}$ ). Unlike for the first order and the third order terms in equations 2 and 3 there is a requirement regarding symmetry for the second order term. This should be noncentrosymmetric, which means that neither the molecules nor the material can have a center of inversion. For glasses, like EO polymers, the dipole moments of the molecules should not add up to zero. 


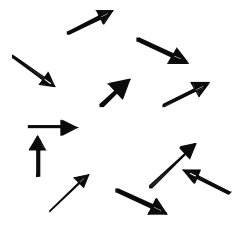

a) Isotropic

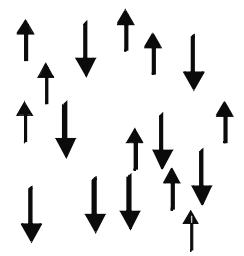

b) Anisotropic

Centrosymmetric

$$
\chi^{(3)} \neq 0
$$

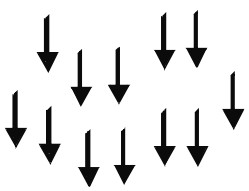

c) Noncentrosymmetric

$$
\chi^{(2)} \neq 0
$$

Figure 2.4: Schematics of isotropic distribution (a), anisotropic but centrosymmetric distribution (b) and non centrosymmetric distribution (c).

This is a very important materials requirement which has to be satisfied in developing second order nonlinear materials. This condition can be easily understood when considering a medium with inversion symmetry. In that case one can write:

$P(-E)=-P(E)$.

Substituting eq. 9 in eq. 3 one gets $\chi^{(2)}=0$. Fig. 2.4 shows the difference between centrosymmetric and noncentrosymmetric media.

We will now consider only the second order nonlinear polarization. This can be written as:

$$
P_{I}\left(\omega_{n}+\omega_{m}\right)=D \sum_{J K} \chi_{I J K}{ }^{(2)}\left(\omega_{n}+\omega_{m} ; \omega_{n} ; \omega_{m}\right) E_{J}\left(\omega_{n}\right) E_{K}\left(\omega_{m}\right)
$$


The factor D, called the degeneracy factor, represents the number of distinct permutations of $\omega_{n}$ and $\omega_{m}$. The first argument of the nonlinear susceptibility is the response

frequency (for example in the case of second harmonic generation this would be $2 \omega$ ) and the second and the third arguments are the frequencies of the incoming E-fields. The process of second harmonic generation is shown in Fig. 2.5.

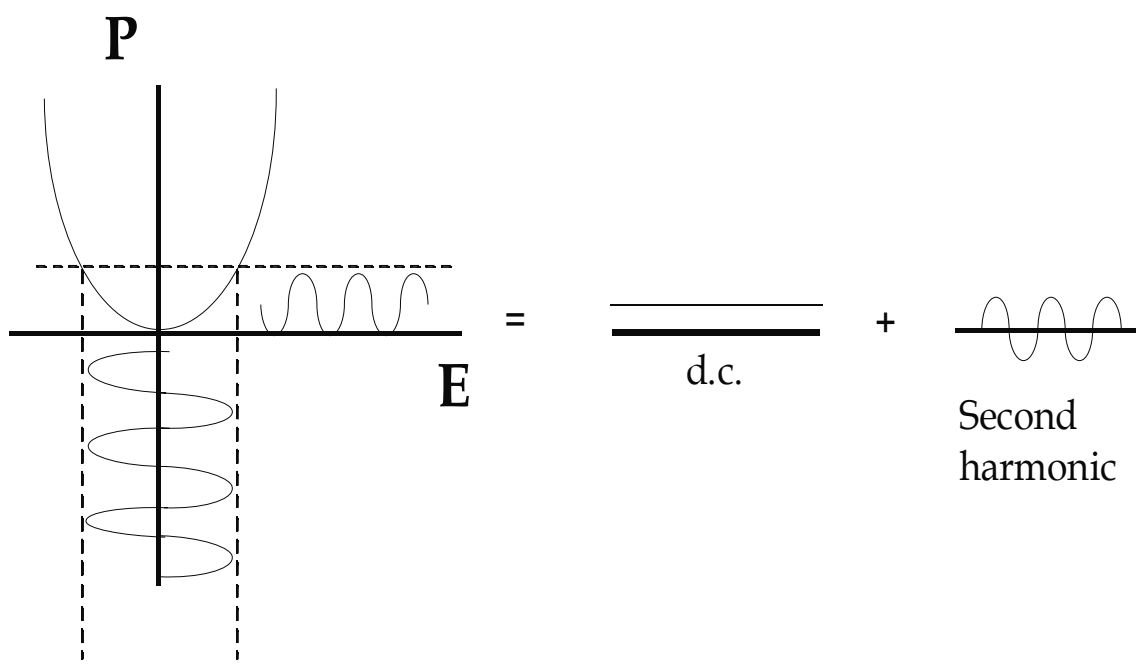

Figure 2.5: A sinusoidal electric field at $\omega$ in a second order nonlinear medium creates a polarization component at $2 \omega$ and a dc component.

It can be seen from equation 10 that each component of the second order nonlinear polarization is given by 9 terms and that the $\chi^{(2)}$ term consequently has 27 elements. By choosing a proper symmetry and applying symmetry conditions the number of independent elements can be reduced. For a lossless medium, that is when the imaginary part of the complex susceptibility is zero, all the frequency arguments can be interchanged as long as the Cartesian indices are changed as well. The sign of the frequency argument must be inverted when the first argument is interchanged with any of the two others.

$$
\chi_{I J K}^{(2)}\left(\omega_{l} ; \omega_{n} ; \omega_{m}\right)=\chi_{J K I}^{(2)}\left(-\omega_{n} ; \omega_{m} ;-\omega_{l}\right)=\chi_{K I J}^{(2)}\left(-\omega_{m} ;-\omega_{l} ; \omega_{n}\right)
$$

The tensor elements are unchanged by permutation of the last two indices and by the permutation of second and third frequency arguments. Therefore,

$$
\chi_{I J K}^{(2)}\left(\omega_{n}+\omega_{m} ; \omega_{n} ; \omega_{m}\right)=\chi_{I K J}^{(2)}\left(\omega_{n}+\omega_{m} ; \omega_{n} ; \omega_{m}\right)
$$


By applying these symmetry operations the number of independent tensor elements is reduced to 18 . Therefore the susceptibility tensor can be represented by $\chi_{I L}{ }^{(2)}$ where $I$ takes the values from 1 to 3 and $L$ varies from 1 to 6 .

In addition to the symmetry properties of the nonlinear susceptibility elements, group theory provides more means to reduce further the number of tensor elements. According to group theory all materials can be classified in one of the possible 32 crystal classes. By invoking $\infty \mathrm{mm}$ symmetry (where the acronyms indicate the fact that the system is invariant under rotation around the surface normal) to which poled polymers belong, their second order susceptibility tensor reduces to

$$
\chi^{(2)}=\left(\begin{array}{cccccc}
0 & 0 & 0 & 0 & \chi_{15}{ }^{(2)} & 0 \\
0 & 0 & 0 & \chi_{15}{ }^{(2)} & 0 & 0 \\
\chi_{31}{ }^{(2)} & \chi_{31}{ }^{(2)} & \chi_{33}{ }^{(2)} & 0 & 0 & 0
\end{array}\right)
$$

When the optical frequencies involved in the nonlinear interaction are far from any resonance effects, the dispersion of the optical response of the material can be ignored. In this case, the tensor elements are unchanged by the permutation of all Cartesian indices without changing the frequency arguments (Kleinman symmetry) as,

$$
\begin{aligned}
& \chi^{(2)}\left(\omega_{n}+\omega_{n} ; \omega_{n} ; \omega_{m}\right)=\chi_{I J K}{ }^{(2)}=\chi_{I K J}{ }^{(2)}=\chi_{J K I}{ }^{(2)}=\chi_{J I K}{ }^{(2)} \\
& =\chi_{K I J}^{(2)}=\chi_{K I J}{ }^{(2)}
\end{aligned}
$$

When Kleinman symmetry is valid, $\chi_{15}{ }^{(2)}=\chi_{31}{ }^{(2)}$ leading to only two independent tensor elements.

\subsubsection{Linear electro-optic effect (pockels effect)}

In the general expression of the second order nonlinear polarization given by equation 10, the frequency arguments of the susceptibility are not limited to optical frequencies and can also take the value zero. In this limiting case when one of the fields involved in the process is a d.c. field (zero frequency or low frequency compared with optical frequencies) the nonlinear polarization describes the process in which the refractive index is modified by an applied electric field. 


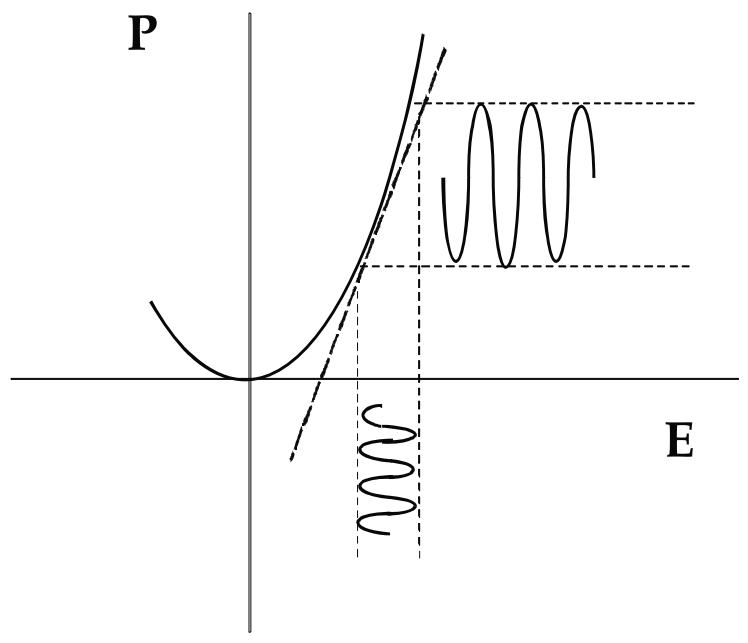

Figure 2.6: Linearization of the second order nonlinear response in the presence of a strong low frequency electric field and weak optical field.

The nonlinear susceptibility tensor for this interaction is of the form $\chi_{I L}{ }^{(2)}(\omega ; \omega ; 0)$. Such a process is known as electro-optic effect. This is schematically shown in Fig. 2.6.

In its broadest sense the electro-optic effect is defined as the change of the refractive index resulting from the application of a d.c. or low frequency electric field. When the index change is proportional to the applied field, it is called the linear electro-optic effect or the Pockels effect.

The refractive index change is given by:

$$
\Delta n=-\frac{1}{2} n^{3} r E(0)
$$

where, $r$ is known as the Pockels coefficient. The medium effectively behaves like a linear optical material with a refractive index of $n+\Delta n(t)$ that is controlled by the electric field $\mathrm{E}(\mathrm{t})$. It is the nonlinear nature of the medium that creates a coupling between the applied electric field and the optical field. Therefore only second order nonlinear media can exhibit a linear Pockels effect. The same symmetry conditions which apply to $\chi^{(2)}$ also apply to $r$ leading to only two independent tensor elements $r_{33}$ and $r_{13}$. These can be related to the second order nonlinear susceptibility by:

$$
\chi_{z z z}^{(2)}(\omega ; \omega ; 0)=-\frac{1}{2} n^{4} r_{33}(\omega ; \omega ; 0)
$$




\subsection{Different classes of NLO polymers}

Different classes of NLO polymers are schematically shown in Fig. 2.7. In the simplest configuration the chromophore is mixed as a guest in the host polymer. The poling efficiency of this system is high as the chromophores are free to rotate without any constraints. The glasstransition temperature $\mathrm{T}_{\mathrm{g}}$ of these kinds of polymers are in general low because of the plasticizing effect of the chromophores. Because of this the polymer can only be doped with a low concentration of chromophore. Typically used chromophore concentration is in the range of $5-10 \mathrm{wt} \%$. This system is in general only used for testing different chromophores and is seldom used for device application.

In the main chain polymer the chromophore is covalently linked within the main chain of the polymer and is a part of the polymer backbone. The poling efficiency of this kind of a polymer is very low as the chromophores are not free to rotate. But the glass transition temperature is very high sometimes even higher than the polymer without chromophores because of hydrogen bonding between the chromophore and the polymer chain.

\section{Guest-host}

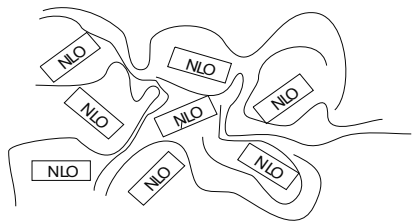

Main Chain

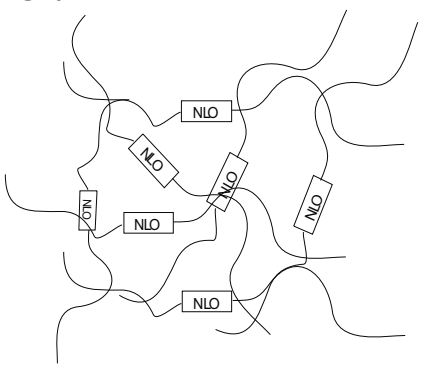

Side chain

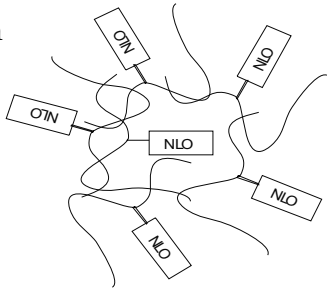

Crosslinked

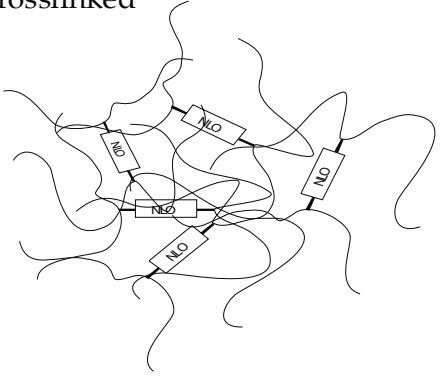

Figure 2.7: Different NLO polymer configurations.

In a side chain polymer the chromophore is attached as a side chain to the polymer backbone. This system combines the advantages of the guest host system and the main chain polymer. A high chromophore concentration can be achieved without lowering much the glasstransition temperature. The poling efficiency is also much better than the main chain polymer. 
In the crosslinked case, the chromophore is initially present as a physical mixture in the polymer. Poling is done first, and the chromophore is fixed in position by crosslinking the polymer at the end of the poling process in the presence of the electric field.

\subsection{Chromophore orientation}

We have already seen that breaking the centrosymmetric arrangement is the basic requirement for second order nonlinearity. This can be done in several ways namely,

1. static field poling

2. photoassisted poling [5]

3. all-optical poling [6].

While the first technique takes advantage of the interaction between the dipole moment and the applied static field, the two others take account of the cooperative effect of static and optical field (photoassisted poling) and purely optical field (all-optical poling). The static field poling can be done in two ways

1. electrode poling

2. corona poling.

In contact electrode poling (Fig. 2.8) the polymer is sandwiched between two electrodes to which a DC voltage is applied. In the case of corona poling the poling field is created by a corona discharge. Each of these techniques has some advantages and disadvantages [7]. In both the cases the film is heated close to its glasstransition temperature (usually slightly below). Corona poling (Fig.2.9) allows application of high poling fields, but often leads to damage of thin films and more particularly severe surface damage, resulting in increased propagation loss after poling. In contact poling the main drawback is the limited voltage that can be applied due to dielectric breakdown $[8,9]$.

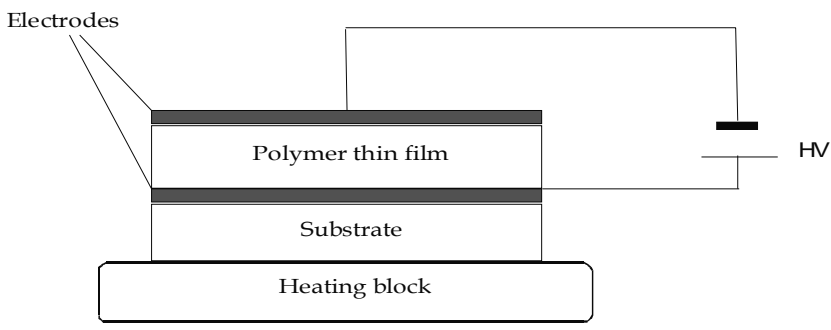

Figure 2.8: Schematic representation of an electrode poling setup.

In the corona poling, the high poling field in the thin film is created by charges deposited on the film. One of the electrodes is a planar electrode on which the film is deposited. The other electrode is usually in the form of a needle. A metal grid is employed between the film and the needle to control the poling current to get a better homogeneity of poling. The applied voltages are in the range of $4-5 \mathrm{kV}$ for a needle at a distance of $1 \mathrm{~cm}$ from the film surface. 
In all cases the chromophore orientation is frozen in by cooling the poled film to room temperature in the presence of the applied field or by thermal- or photo-crosslinking during poling.

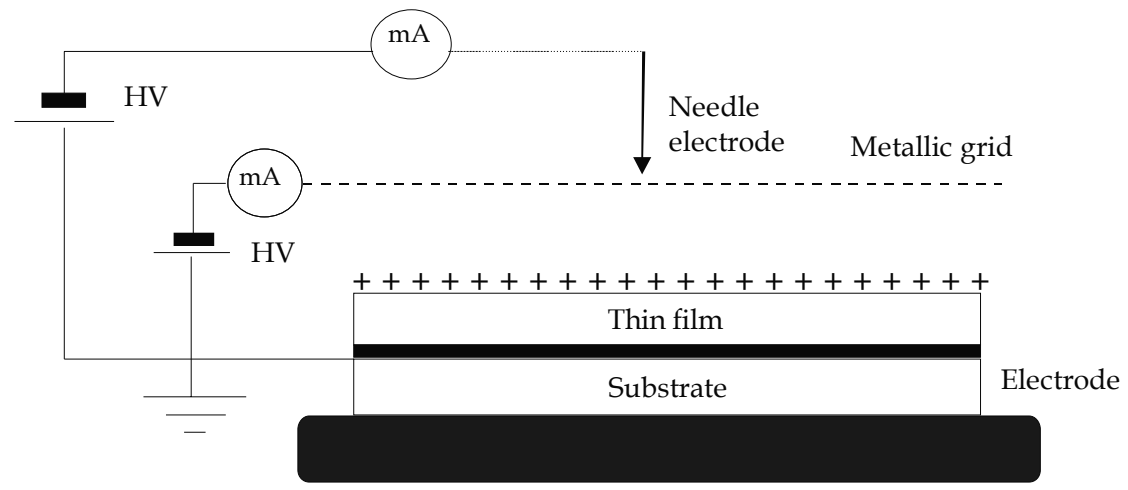

Figure 2.9: Schematic representation of a corona poling setup.

Assuming that $\beta_{z z z}$ along the charge transfer direction is the dominant factor, one obtains:

$\chi_{z z z}^{(2)}=N \beta_{z z z}\left\langle\cos ^{3} \theta\right\rangle / 2$

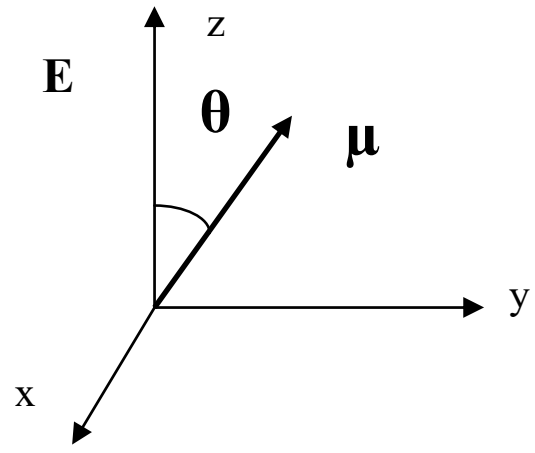

Figure 2.10: Molecular axis orientation with respect to the poling field $E$ aligned in z-direction.

Where $N$ is the chromophore number density and $\left\langle\cos ^{3} \theta\right\rangle$ is the angular average, which describes the polar order of the molecules, called the order parameter. The driving force for the orientation of the chromophores is the interaction between the ground state 
dipole moment $\mu$ and the poling field. The molecules try to orient in the direction of the applied field in order to reduce their energy $\vec{\mu} \cdot \mathbf{E}$ (see Fig. 2.10). The mean orientation of the chromophores in an electric field is due to the competition between the orientation energy $\vec{\mu} \cdot \mathbf{E}$ and the thermal disorientation energy $k_{B} T$, with $T$ the poling temperature.

\subsection{Material requirements for an electro-optic modulator}

A schematic of EO Mach-Zhender modulator is shown in Fig. 2.11. For fabricating an electro-optic modulator, the properties of three different kinds of materials have to be taken into account.

1. electro-optic chromophore

2. chromophore containing polymer

3. polymer cladding

Top view
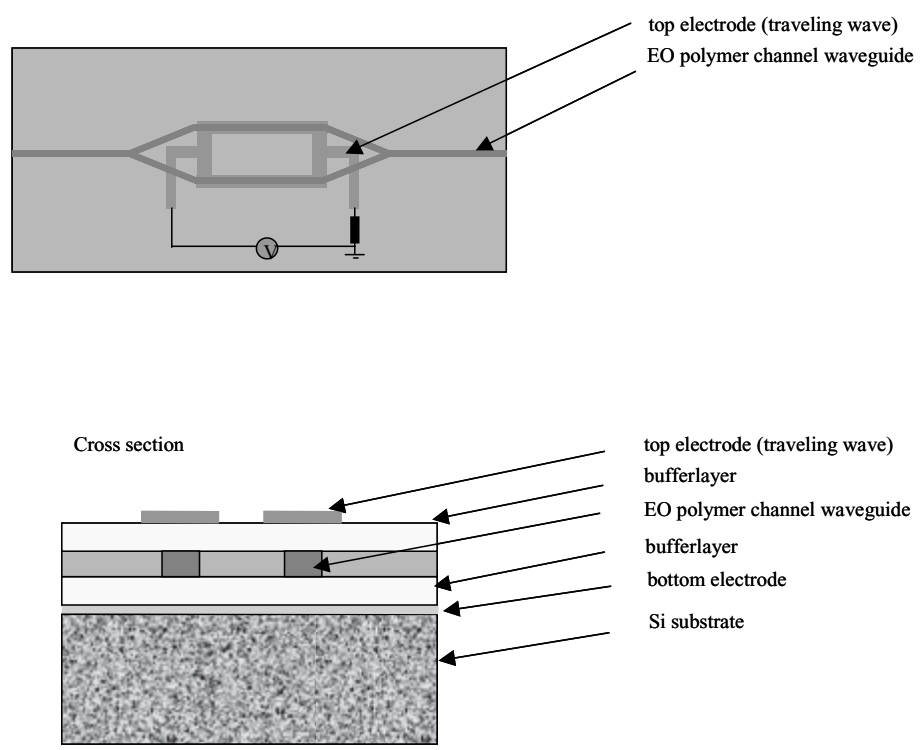

top electrode (traveling wave) bufferlayer

EO polymer channel waveguide

bufferlayer

bottom electrode

Si substrate

Figure 2.11: Schematic of an EO polymer Mach-Zhender modulator. 
In the first case a suitable chromophore has to be found. For application in optical communication, devices characterized by drive voltages of about $1 \mathrm{~V}$ are required. There are also some few applications such as cable TV that do not require such low voltage operation. However, in general sub $1 \mathrm{~V}$ operation is requested. In order to be competitive with the currently available Lithium Niobate modulators, an electro-optic coefficient in the order of $30 \mathrm{pm} / \mathrm{V}$ is required. Table 2.2 shows some of the state-of-the-art chromophores and their $\mu \beta$ values. The figure of merit of a chromophore is given by $\mu \beta / M_{w}, M_{w}$ being the molecular mass of the chromophore.

Table 2.2: Different chromophores and their properties.

\begin{tabular}{|c|c|c|c|c|}
\hline NLO chromophore & & $\begin{array}{l}\lambda_{\max } \\
(\mathrm{nm})\end{array}$ & $\begin{array}{l}\mu \beta \\
\left(10^{-48} \mathrm{esu}\right)\end{array}$ & $\mu \beta / \mathrm{M}_{\mathrm{w}}$ \\
\hline & DR1 & 475 & 800 & 3.0 \\
\hline & DANS & 438 & 580 & 2.1 \\
\hline & TCVDPA & 531 & 584 & 1.7 \\
\hline TBDMS- 0 & CLD & 695 & 35000 & 45.7 \\
\hline & FTC & 650 & 18000 & 25.9 \\
\hline
\end{tabular}

Chromophores to be used in electro-optic application should not exhibit significant optical absorption at telecommunication wavelengths of $1.3 \mu \mathrm{m}$ and $1.5 \mu \mathrm{m}$. In most cases vibration induced absorptions from $\mathrm{C}-\mathrm{H}$ overtones will be dominant at telecommunication wavelengths. Vibrational absorption by the chromophore will, in general, not pose a serious problem for the device operation, although it can contribute to a non-negligible fraction of the total losses at very high chromophore concentration. Normally the host polymer will have a higher concentration of protons than the chromophore and will constitute a greater weight fraction of the final material. Thus the vibrational absorption of the polymer host will be the largest contribution. Optical losses 
arising from such absorption from the host polymer is typically in the order of $1 \mathrm{~dB} / \mathrm{cm}$ at $1.3 \mu \mathrm{m}$ and slightly higher at $1.5 \mu \mathrm{m}$.

The most important problem associated with the optical absorption of the chromophores is with the interband electronic excitation. This typically should not be a problem at telecommunication wavelengths if the $\lambda_{\max }$ of the chromophore if below $700 \mathrm{~nm}$. However the host polymer can influence this property of the chromophores if there are chromophore aggregations because of matrix incompatibility.

Chromophores must be thermally robust enough to withstand temperatures encountered during electric field poling and processing of the chromophore-polymer material. Thermal stability is defined as the temperature at which decomposition is first observed. Chromophore decomposition temperatures can be determined by thermal gravimetric (TGA) and differential scanning calorimetry (DSC). These measurements will yield decomposition temperatures lower than those for the same chromophore in the hardened polymer matrix. To be useful for the development of device quality material, a chromophore must exhibit thermal stability of $250^{\circ} \mathrm{C}$ or higher.

Chromophores must also exhibit chemical, electrochemical, and most importantly, photochemical stability at the operating wavelength [10 - 13]. The chromophore degrades at the operating wavelength because of singlet oxygen attack. Most of the high $\mu \beta$ chromophores are prone to this kind of photochemical degradation. It had for long been very difficult to meet these two requirements of high $\mu \beta$ and photochemical stability.

The chromophore and the host polymer should have comparable solubility in spin coating solvents. The preparation of optical quality films requires a balance between solution viscosity and solvent volatility. Finally the chromophore must have a shape and segment flexibility appropriate for efficient poling. In the next chapter we will discuss, at length, the choice of the shape that minimizes the unwanted intermolecular interactions. This is extremely important in maximizing the macroscopic electro-optic activity. The chromophore must also be compatible with the matrix in which it is taken up. Phase separation quickly leads to unacceptably high optical losses due to scattering from microdomains.

The host polymer should exhibit good thermal stability, low optical absorption and good solubility in spin coating solvents. The glasstransition temperature of the host polymer should be sufficiently high (at least about $100^{\circ} \mathrm{C}$ higher than the temperatures encountered during operation) to impede chromophore relaxation after the poling field is turned off and the material is returned to room temperature. The difference between the room temperature and the glass transition temperature determines the temporal stability of the poling order $[14,15]$. The presence of chromophores will produce some plastization of the host polymer, particularly if the chromophore is not covalently linked to the polymer but rather simply physically incorporated into the polymer. Thus the glass transition temperature of the polymer should be in the range of $150^{\circ} \mathrm{C}$ to $200^{\circ} \mathrm{C}$. For temperatures higher than this, sublimation of chromophores could be a problem during poling. To avoid electrical conductivity that will attenuate the poling voltage felt by the chromophores, both the chromophore and the polymer should be free of ionic impurities $[16,17]$. 
A cladding material is required for preventing the optical wave propagating in the core material to sense the optically lossy metal electrodes used for applying the electric modulating field. An electro-optic device consists of different layers stacked on each other (substrate, bottom electrode, bottom cladding, core layer, top cladding and top electrode, see-Fig. 2.11). To find a suitable cladding material which is compatible with the core layer becomes a very important requirement.

In general cladding materials have larger electrical conductivity than that of the active polymer layer [18 - 20]. Such conductivity permits the applied electric field to be reduced across the cladding layer yielding a greater field felt by the chromophores in the active layer. This is essential for achieving improved poling efficiency. The cladding material should have low optical loss and its refractive index should be lower than the active polymer layer to confine the optical mode in the active layer. The cladding layer should be compatible with and exhibit good adhesion with the polymer layer. Spin coating solvents used for deposition of the cladding layer should not dissolve the active layer. Such solvent damage leads to very high optical losses. The glasstransition temperatures of the cladding and the active polymer must be comparable in order to avoid thermal stresses during poling. UV curable epoxies are most commonly used cladding materials. In that case the active material must be capable of withstanding the required UV dose for the cladding. The cladding material must be thermally and photochemically stable. Degradation of the cladding material can lead to mode expansion and evolution of the waveguide from single mode to bimodal.

The electrode used for applying the electric field has a dramatic effect on the device performance parameters such as bandwidth, drive voltage and optical losses. The microwave losses can be attributed to the dielectric losses of the polymer, electrode resistive losses and phase mismatch between the optical and electrical wave. The dielectric losses of the polymers and the phase mismatch issues are of less concern in the case of polymers and are not considered as design parameters. The main deciding factor at high frequencies $(100 \mathrm{GHz})$ is the electrode losses. For frequencies beyond a few $\mathrm{GHz}$, the electrode should be designed in the traveling wave configuration. For lower frequencies the electrode can still be used as a lumped element.

As a consequence of the above, a number of often conflicting requirements must be simultaneously satisfied when choosing different materials in order to arrive at a successful electro-optic device.

\subsection{Processing and fabrication}

\subsubsection{Spin coating}

With spin coating the following issues are of concern.

1. volatality of the spin coating solvent

2. complete solubility of the chromophore and the host polymer 
3. viscosity of the solution

Higher molecular weight polymers and crosslinked polymers exhibit reduced solubility in commonly used solvents. The solvent should have a low boiling point so that it easily can be driven out of the film after spinning. Usually spin coating solvents have boiling temperatures around $100^{\circ} \mathrm{C}$. A problem with low volatility solvents is the trapping of solvents in the spin-cast film. Such solvent inclusions can lead to light scattering and high losses. Moreover solvent inclusion can be a source of thermal instability during processing and device operation as the solvents which gas out through the top cladding from beneath leaves craters on the surface and could even damage the top electrode.

The chain length of the polymer is an important factor for making optical quality films. Short chain lengths favor homogeneous spinning and planarization. On the other hand, a long chain length favors lattice rigidity which is required for retention of the poling induced order. Long chain length also pose solution filtering problems when using $0.2 \mu \mathrm{m}$ pore filters and may require the use of large area spiral cap filters. Therefore it would be ideal to have low molecular weight and flexible polymers during spin coating and end up with a hardened polymer matrix after poling. To control the homogeneity of the film during spinning without inclusion of dust particles is of extreme importance. The reasoning is twofold. These particles not only act as scattering sites, but also during poling the refractive index contrast around such inhomogeneities (poling insensitive) region increases. In this way poling induced scattering sites are created.

\subsubsection{Fabrication techniques}

Two mainly used fabrication techniques for the fabrication of waveguides in polymers are

1. reactive ion etching

2. photodefinition.

Reactive ion etching in polymers is not straight forward in comparison with inorganic materials. Because of the edge beading (formation of uneven surface at the edge of the wafer during spinning) caused during spinning, establishing good contact with the mask becomes extremely difficult. This limits the maximum resolvable feature size to be $2 \mu \mathrm{m}$ using standard contact UV lithography. Reactive ion etching induces surface roughness and particularly side wall roughness which increase scattering losses. Photodefinition using photodefinable polymers on other hand offers the possibility to resolve $800 \mathrm{~nm}$ structures and also results in smooth side walls. The main problem with photodefinable polymers is their poor poling property because of the presence of highly conducting species required for the generation of the photo acid.

\subsection{Device configurations}

Most electro-optic modulators are made using a Mach-Zehnder (MZ) interference configuration, see Fig. 2.11. A MZ interferometer has a sinusoidal response. In the current 
work we make use of microring resonators for electro-optic modulation. The response of a microring resonator has a Lorentzian shape so that large amplitude modulation can be achieved with low voltages. Also, microrings have the advantage of dense integration and compactness because of their small size. For the refractive index contrast available between the cladding and core polymer, the ring radius lies in the range of $100 \mu \mathrm{m}$ to 200 $\mu \mathrm{m}$ depending on the design. Because of such small dimensions the electrode losses will also be less compared with $\mathrm{cm}$ long electrodes required for MZ.

\subsection{References}

[1] L. Dalton, Pure Appl. Chem., 76, p1421, 2004.

[2] H. S. Nalwa, S. Miyata, Nonlinear Optics of Organic Molecules and Polymers, CRC Press, 1997.

[3] L. A. Hornak Polymers for Lightwave and Intergrated Optics, 1992.

[4] S. K. Kim, L. Xue, IEEE Electron Device Lett., 28, p706, 2007.

[5] Z. Sekkat, M Dumont, Nonlinear Opt., 2, p359, 1992.

[6] F. Charra, F. Kajzar, J. M. Nunzi, P. Raimond, E. Idiart, Opt. Lett., 18, p941, 1993.

[7] F. Kajzar, J. M. Nunzi, Molecule orientation techniques, p101, 1998.

[8] M. Sprave, R. Blum, M. Eich, Appl. Phys. Lett., 69, p2962, 1996.

[9] R. M. Blum, J. Sablotny, M. Eich, Opt. Soc. Am., 15, p318, 1998.

[10] Q. Zhang, M. Canva, G. I. Stegeman, Appl. Phys. Lett.,73, p912, 1998.

[11] A. Dubois, M Canva, A. Brun, F. Chaput, J. P. Boilot, Appl. Opt., 35, p 1996, 1996.

[12] C. Cai, I. Liakatas, M. S. Wong, C. H. Bosshard, P. Gunter, Polym. Prepr., 39, p1111, 1998.

[13] A. Galvan-Gonzalez, K. D. Belfield, G. I. Stegeman, K. P. Canva Chan, K. Paek, L. Sukhomlinova, R. J. Twieg, Appl. Phys. Lett., 77, p2083, 2000.

[14] M. Faccini, M. Balakrishnan, M. B. J. Diemeer, Z. P. Hu, K. Clays, I. Asselberghs, A. Leinse, A. Driessen, D. N. Reinhoudt, W. Verboom, J. Of Mater. Chem., 18, p2141, 2008.

[15] Y. M. Cai, A. K. Y. Jen, Appl. Phys. Lett., 67, p299, 1995. 
[16] L. R. Dalton, A.W. Harper, B. Wu, R. Goshn, J. Laquindanum, Z. Liang, A. Hubbel, C. Xu, Adv. Mater., 7, p519, 1995.

[17] L. R. Dalton, W. H. Steier, B. H. Robinson, C. Zhang, A. Ren, S. Garner, A. Chen, T. Londergan, L. Irwin, B. Carlson, L. Fifield, G. Phenlan, C. Kincaid, J. Amend, A. Jen, Mater. Chem., 9, p1905, 1999.

[18] G. Gadret, F. Kajzar, P. Raimond, Proc. SPIE, 1560, p226, 1991.

[19] T. Dantas de Morais, C. Noel, F. Kajzar, Nonlinear Opt., 15, p315, 1996.

[20] D. Gonin, B. Guichard, M. Large, T. Dantas de Morais, C. Noel, F. Kajzar, Nonlinear Opt. Phys. Mater., 5, p735, 1996. 
3 characterization and optimizing the properties of the TCVDPA chromophore for application in EO modulators

This chapter describes the properties of the TCVDPA chromophore and its modifications. The poling procedure has been optimized and the EO coefficient has been measured. Enhancement in the poling efficiency could be obtained by steric modification of the TCVDPA chromophore with different bulky groups.

Published as: M. Balakrishnan et al, Electronics Letts, 42 (1), PP 51-52, 2006. 


\subsection{Introduction}

Organic materials exhibiting large electro-optic responses $\left(r_{33}\right)$ have attracted considerable attention over the past two decades [1, 2]. They have great potential for use in telecommunication, information processing, phased array radar, optical storage devices, $\mathrm{THz}$ generation, and many other applications as active materials in photonic micro devices. The advantages of organic materials over traditional inorganic materials such as $\mathrm{LiNbO}_{3}$ are high and fast nonlinearities, ease of processing and device integration and the possibility of structural modification depending on the desired application [3,4]. In addition, because of the low dielectric constant of polymers at RF frequencies, optical and RF fields can easily be phase matched in a traveling wave arrangement so that efficient modulation beyond $100 \mathrm{GHz}$ could be demonstrated [5].

However, having high nonlinearity is not enough to ensure wide scale commercial utilization of polymeric electro-optic devices. Other essential properties, such as good thermal, mechanical and photochemical stability, low optical loss (high transparency) and good processability, need to be simultaneously optimized in order to be successfully implemented in a practical device.

Several classes of compounds exhibiting very high thermal stability (above $300^{\circ} \mathrm{C}$ ) have been reported [6-8]. One of the most crucial parameters, however, for the long term efficiency of EO devices is photostability [9-13], since they are expected to last for years without significant degradation under high photon flux. The absorption of photons by molecules under illumination can lead to changes in their chemical structure and consequent loss of nonlinearity. It is necessary that the absorption band of the chromophore lies far from the operating wavelength, which in case of communication devices is at $1300 \mathrm{~nm}$ and $1550 \mathrm{~nm}$. Apart from this, an even more serious issue is the generation of singlet oxygen at these wavelengths that attacks the chromophore. Several research groups have done extensive work examining the photostability of EO polymers. They studied the influence of factors such as the chromophore structure, wavelength and intensity, and the presence of oxygen. The general conclusion that can be drawn from these studies is that the most photostable compounds have a simple benzene $\pi$-bridge and a tricyanovinyl electron-acceptor group. More elongated conjugated bridges between the electron-donor and -acceptor groups lead to faster photodegradation. GalvanGonzalez et al. $[9,12]$ identified tricyanovinylidenediphenylaminobenzene (TCVDPA) as the most photostable structure: it is about two orders of magnitude more stable than the DANS (4- $N, N$-dimethylamino-4'-nitrostilbene) chromophore and one order of magnitude more stable than the azo chromophore DR1 (4-[N-ethyl-N-2-hydroxyethyl)amino]-4'nitroazobenzene) [9]. In fact, it shows no degradation upon irradiation at the absorption maxima $\left(\lambda_{\max }\right)$, nor it is acting as a sensitizer for the formation of singlet oxygen upon radiation with $U V$. On the other hand, the price to pay for this higher stability is a shorter conjugation path and therefore a lower second-order nonlinearity compared to extremely large chromophores like CLD or FTC (see Table 1.2). The photostability of such chromophores, however, has been reported to be quite poor in air [13]. Consequently high-cost packaging is necessary for shielding the EO devices from oxygen in the air, which is responsible for the photodegradation of the chromophore by the formation of singlet oxygen. Given that photostability is of key importance to the long-term reliability 
of devices, TCVDPA holds a strong competitiveness for EO applications. Moreover, the TCVDPA chromophore possesses the unique feature of having a low absorption window in the UV region (see Fig. 3.2), where most of the chromophores absorb, in this way allowing UV-crosslinking for photodefinition.<smiles>N#CC(C#N)=C(C#N)c1ccc(N(c2ccccc2)c2ccccc2)cc1</smiles>

Figure 3.1: Chemical structure of the TCVDPA chromophore.

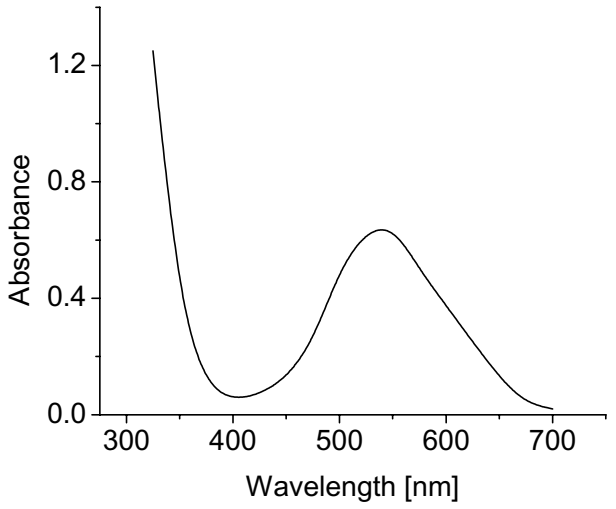

Figure 3.2: Absorption spectrum of TCVDPA showing a low UV absorption window.

\subsection{Properties of the TCVDPA chromophore}

\subsubsection{Refractive index measurements}

For the refractive index measurements TCVDPA chromophores were incorporated in SU8 (a negative photoresist from Microchem ${ }^{1}$ ) as guest-host $(\mathrm{GH})$ polymer.

${ }^{1}$ WWw.microchem.com 
Films with different concentrations of TCVDPA, $5 \mathrm{wt} \%, 10 \mathrm{wt} \%$ and $20 \mathrm{wt} \%$ were prepared and the refractive index (n) was measured using a Woolam ellipsometer. Fig. 3.3 shows the refractive index of SU8 as a function of the TCVDPA concentration. A linear behavior is observed up to a concentration of $20 \mathrm{wt} \%$. This is in indication that chromophore segregation does not take place up to $20 \mathrm{wt} \%$ of TCVDPA in SU8. This relatively high chromophore concentration is made possible by taking advantage of the fact that SU8 is in the uncrosslinked monomeric state. This is attractive as high concentrations of chromophore can be achieved without segregation problems, which in turn will lead to high nonlinearity, as the NLO properties scale with the chromophore number density. The small deviation from linear behavior at $5 \mathrm{wt} \%$ is a measurement error caused by the film thickness that was much larger than the optimum value required by the Woolam ellipsometer for accurate measurement of refractive indices in this refractive index range.

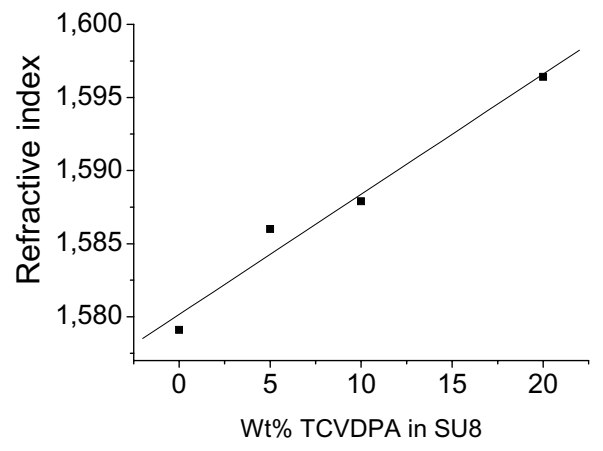

Figure 3.3: Refractive index of SU8 as a function of TCVDPA concentration.

\subsubsection{Photodefinition by UV-thermal crosslinking}

Photodefinition of polymers is an attractive fabrication technique for channel waveguides. It is an elegant method requiring a minimum of processing steps when compared to reactive ion etching (RIE) with the need of a patterned resist layer. Photodefinition of SU8-TCVDPA guest-host polymer is demonstrated by exploiting the low UV absorption window of the TCVDPA chromophore shown in Fig. 3.2. In this wavelength range most of the other chromophores are highly absorbing. Fig. 3.4 shows a photo defined channel in SU8 $15 \mathrm{wt} \%$ TCVDPA on Si substrate. The doped SU8 layer was spun on Si at $3000 \mathrm{rpm}$ to give a film thickness of $2 \mu \mathrm{m}$. It was then baked at $95^{\circ} \mathrm{C}$ for $5 \mathrm{~min}$ on hot plate (pre bake). The polymer film was then exposed to UV with a bright field $\mathrm{Cr}$ mask for $5 \mathrm{~min}$ and cured at $95^{\circ} \mathrm{C}$ for $5 \mathrm{~min}$ (post bake). The dark regions in Fig. 3.4 are crosslinked and SU8 in the bright channel was uncrosslinked and could be removed during development. 
Besides the mask pattern the channel geometry depends on the exposure time and the post bake time. These parameters can be optimized to obtain perfect side walls. A more detailed explanation of the photodefinition process is presented in chapter 5 .

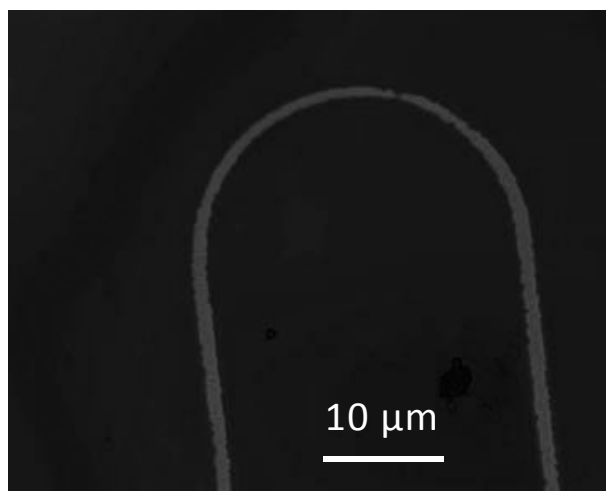

Figure 3.4: Optical microscope picture of a phooligined channel in SU8-15 wt\% TCVDPA. The radius of the curved section is $15 \mu \mathrm{m}$.

\subsubsection{Functionalization of TCVDPA with different bulky groups}

The TCVDPA chromophore can be functionalized with bulky side groups as shown in Fig. 3.5. This bulkiness will inhibit closer approach of the chromophores and thereby prevent antiparallel clustering of the chromophores during poling. When equipped with epoxy molecules as side groups, the chromophores can be anchored to the polymer backbone (in the case of SU8 host) which will improve temporal stability and also photochemical stability to some extent. But the main function of these bulky groups would be to reduce the intermolecular interactions during electric field poling. Bulky groups with varying degree of bulkiness were chosen and attached to the donor side of the chromophore as shown in Fig. 3.5. 
<smiles>N#CC(C#N)=C(C#N)c1ccc(N(c2ccccc2)c2ccccc2)cc1</smiles>

C1 (TCVDPA)<smiles>COc1ccc(N(c2ccc(OC)cc2)c2ccc(C(C#N)=C(C#N)C#N)cc2)cc1</smiles>

C2<smiles>CC(C)(C)c1ccc(N(c2ccc(C(C#N)=C(C#N)C#N)cc2)c2ccc(C(C)(C)C)cc2)cc1</smiles>

C3<smiles>CCC(CC)COc1ccc(N(c2ccc(OCC(CC)CC)cc2)c2ccc(C(C#N)=C(C#N)C#N)cc2)cc1</smiles>

C4<smiles>N#CC(=C(N)/C(C#N)=C(/C#N)c1ccc(N(c2ccc(OCc3c(F)c(F)c(F)c(F)c3F)cc2)c2ccc(OCc3c(F)c(F)c(F)c(F)c3F)c(C(=O)Oc3ccc(N(c4ccc(OCc5c(F)c(F)c(F)c(F)c5F)cc4)c4ccc(OCc5c(F)c(F)c(F)c(F)c5F)cc4)cc3)c2)cc1)c1ccc(N(c2ccc(OCc3c(F)c(F)c(F)c(F)c3F)cc2)c2ccc(OCc3c(F)c(F)c(F)c(F)c3F)cc2)cc1</smiles>

C5

C6

Figure 3.5: TCVDPA modified with bulky groups; C1: unmodified, C2: Methoxy, C3: butyl, C4: alkyl, C5: fluorinated benzene, C6: fluorinated benzene in the form of dendrites.

The effect of these different bulky groups in reducing the intermolecular interaction during poling will be discussed later on in this chapter. These bulky groups only provide bulkiness to the chromophore without influencing the $\beta$ value of the chromophore in all cases except C6. The measured $\beta$ values of these different chromophores are presented in Table 3.2.

\subsubsection{Optical Loss Measurements}

Optical losses were measured by the prism coupling method [14] and the results are shown in Figure 3.6. Slab waveguides were made by depositing a polymer film on $8 \mu \mathrm{m}$ thick silicon oxide on silicon wafers by spin coating. The films were made with two 
different concentrations of $\mathrm{C} 1$ (20 wt \% and $30 \mathrm{wt} \%)$ in polysulfone (PS). For reference a PS film without $\mathrm{C} 1$ was also made. The resulting film thickness was about $4 \mu \mathrm{m}$ and the waveguide structure sustains three modes at $1550 \mathrm{~nm}$. As the surface of the spin coated film is extremely smooth, the loss mechanism is mainly due to materials absorption. White light from a broadband source is coupled into the polymer slab using a prism. After propagating a certain distance it is out-coupled using another prism and sent to a spectrum analyzer. The experiment is repeated varying the distance between the incoupling and the out-coupling prisms. By plotting the out-coupled power as a function of the distance of propagation it is possible to obtain the absorption loss spectra.

In general, device-quality EO materials should possess good optical transparency (low optical loss), in particular at the main telecom wavelengths $(1310 \mathrm{~nm}$ and $1550 \mathrm{~nm})$ and datacom wavelengths $(840 \mathrm{~nm})$. For the PS host polymer the optical loss remains relatively low (around $1 \mathrm{~dB} / \mathrm{cm}$ ) at $840 \mathrm{~nm}, 1310 \mathrm{~nm}$ and at $1550 \mathrm{~nm}$. When $20 \mathrm{wt} \%$ of chromophore C1 is incorporated as guest into a PS host matrix, the long-wavelength tail of the main absorption peak of the chromophore at $539 \mathrm{~nm}$ extends far into the near-IR and therefore causing a high loss of $7.2 \mathrm{~dB} / \mathrm{cm}$ at $840 \mathrm{~nm}$. Although the high chromophore loading causes strong vibrational $\mathrm{C}-\mathrm{H}$ overtone absorption, it has no detrimental effect on the optical transparency at telecom wavelength. As these losses only slightly increase to 1.7 and 1.5 at $1310 \mathrm{~nm}$ and $1550 \mathrm{~nm}$, respectively, the material appears to be well suitable for waveguide applications at telecom wavelengths.

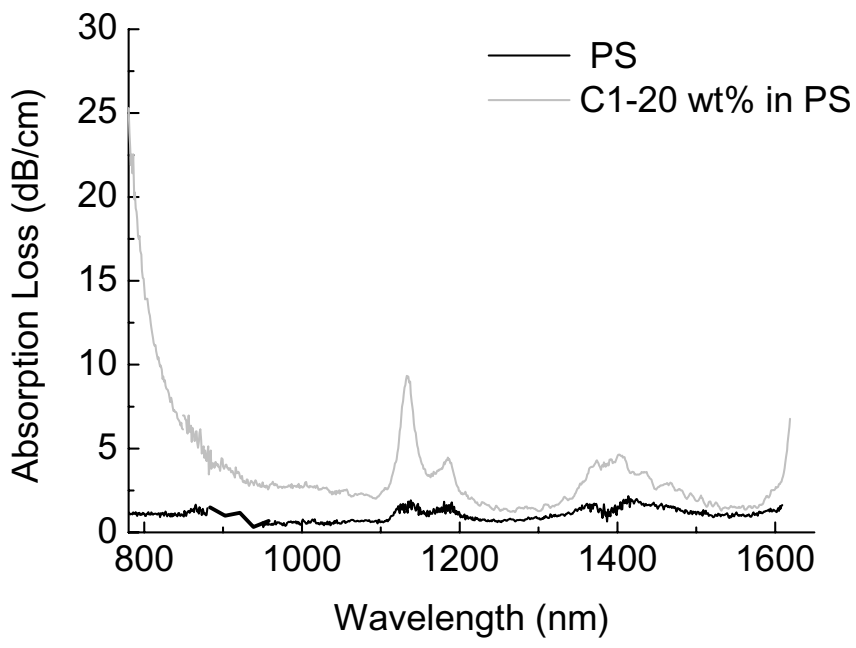

Figure 3.6: Optical loss spectrum of PS and PS-20 wt\% C1 slab waveguides.

The loss values measured at single laser wavelengths are shown in Fig. 3.6a. 


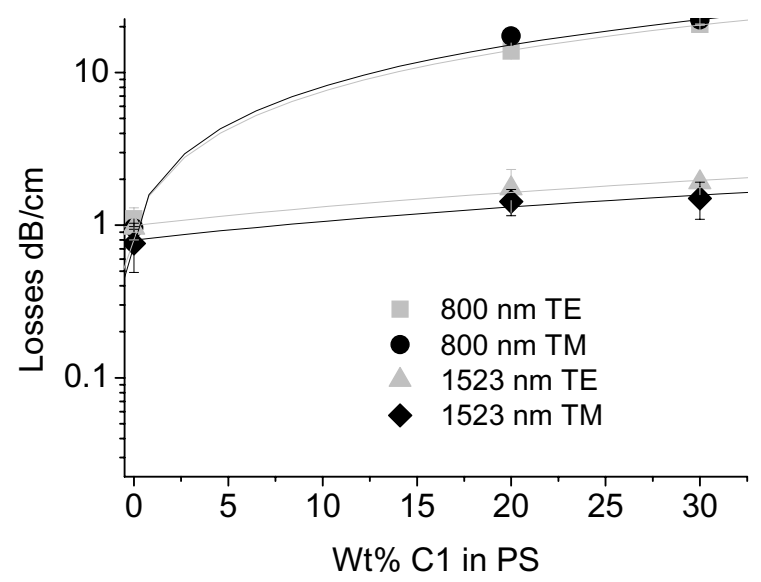

Figure 3.6a: PS-TCVDPA slab waveguiding losses.

\subsubsection{Thermal properties of TCVDPA}

Thermal stability is a critical parameter for long term efficiency of EO devices. Since the NLO response has to be stable during processing and operation of the chromophore/polymer materials, the chromophores need to be chemically stable at all temperatures that the system encounters in electric field poling. In addition they should withstand all fabrication steps needed for device fabrication.

The thermal properties of the chromophores are reported in Table 3.2. Thermal Gravimetric Analysis (TGA) data were recorded at a heating rate of $20^{\circ} \mathrm{C} / \mathrm{min}$. It should be noted that weight loss in these experiments may be due to sublimation and/or decomposition of the substance. The decomposition temperature of chromophores C1-C6 is really high, being above $320^{\circ} \mathrm{C}$ for all of them. The highest value, $365^{\circ} \mathrm{C}$, which has been recorded for $\mathrm{C} 6$, is among the highest ever reported for NLO chromophores. The weight loss decomposition temperature, $T_{d}{ }^{5}$, is defined as the point at which $5 \%$ weight loss has occurred in the chromophore. $T_{d}$ onset is calculated from the intersection of the tangent to the slope of the curve corresponding to the first weight loss event which is normally at higher temperatures than $T_{d}{ }^{5}$. The wavelength of maximum absorption, $\lambda_{\max }$, was measured in $\mathrm{CH}_{2} \mathrm{Cl}_{2}$ solution.

The Hyper-Rayleigh scattering (HRS) technique was employed to measure $\beta_{z z z}$ for all chromophores at $800 \mathrm{~nm}$ (Table 3.2). In most of the cases, the hyperpolarizability is quite constant, slightly above $400 \times 10^{-30} \mathrm{esu}$. Apparently, the functionalization at the donor site of the chromophores does not have a large influence on $\beta_{z z z}$. However, the sensibly lower 
value found for chromophore C6 may be due to the ester bond directly connected to the diphenylamine moiety, reducing its electron donating ability.

Table 3.2: Summary of thermal and optical properties of chromophores C1-C6.

\begin{tabular}{lllll}
\hline & $T_{d}^{5}$ & $T_{d}$ onset & $\lambda_{\max }$ & $\beta_{z z z}$ \\
& $\left({ }^{\circ} \mathrm{C}\right)$ & $\left({ }^{\circ} \mathrm{C}\right)$ & $(\mathrm{nm})$ & $\left(10^{-30} \mathrm{esu}\right)$ \\
\hline $\mathrm{C} 1$ & 286 & 335 & 539 & 425 \\
$\mathrm{C} 2$ & 331 & 530 & 428 \\
$\mathrm{C} 3$ & 296 & 323 & 537 & 424 \\
$\mathrm{C} 4$ & 292 & 360 & 545 & 418 \\
$\mathrm{C} 5$ & 350 & 326 & 536 & 409 \\
$\mathrm{C} 6$ & 308 & 365 & 529 & 275
\end{tabular}

\subsubsection{Photobleaching experiments}

Several research groups have done extensive work examining the photostability of EO polymers. Previous studies clearly show that the presence of oxygen can greatly increase the degradation rate of organic chromophores [15]. In these studies the photobleaching measurements were made by using lasers as a light source on film samples of guest-host polymeric materials.

In our case, photobleaching tests were carried out for chromophores $\mathrm{C} 1, \mathrm{C} 3$, and $\mathrm{C} 5$ and for the commercially available NLO chromophores DANS and DR1 by monitoring the decrease in absorbance (A) during irradiation of oxygen-saturated solutions of chromophores in $\mathrm{CDCl}_{3}$ with visible white light. After 100 mins absorbance of $\mathrm{C} 1, \mathrm{C} 2$ and C3 reduces by only about $2 \%$, whereas the absorbance of DR 1 reduces by about $20 \%$. Our data compare well with literature where TCVDPA has "an order of magnitude" higher photostability than some other representative EO chromophores (Table 3.3). The stilbene chromophore DANS degrades very rapidly due to the attack on the central carbon double bond by oxygen. The most photostable compounds are characterized by benzene bridges and tricyanovinyl electron-acceptor groups. 


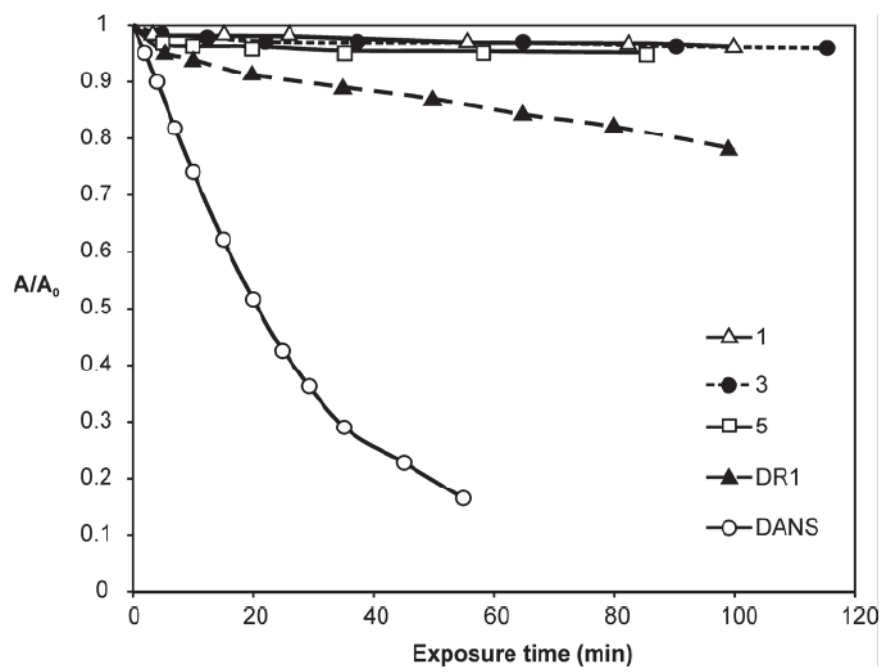

Figure 3.7: Photo-bleaching curves of chromophores C1, C3, C5, DR1, and DANS in solution. Shown is the ratio of $A / A_{0}$ as a function of exposure time; $A$ is the absorbance at time $t$ and $A_{0}$ is the initial absorbance.

From the results shown in Fig. 3.7 it can be noticed that chromophores C1, C3 and C5 are photostable under our experimental conditions, showing hardly any degradation upon exposure to white light for $120 \mathrm{~min}$. Moreover, comparing the decay curves of chromophores C3 and C5 with that of C1, no significant difference can be noticed. More than $95 \%$ of the initial absorbance is retained after $100 \mathrm{~min}$ of exposure, showing that functionalization with bulky side groups does not have a detrimental influence on the photostability. From these data it can be concluded that chromophores C1-C6 possess among the highest photostabilities reported for D-л-A chromophores. In the experiments described in Chapter 5 we observe excellent photostability in our EO devices made by SU8-C1. 
Table 3.3: Photostability data of TCVDPA, DANS and DR1 chromophores from literature [16]. B is the number absorption events needed, on average to photodegrade a single chromophore molecule.

\begin{tabular}{|c|c|c|c|c|c|}
\hline NLO chromophore & & $\begin{array}{l}\lambda_{\max } \\
(\mathrm{nm})\end{array}$ & $\begin{array}{l}\mu \beta \\
\left(10^{-48} \mathrm{esu}\right)\end{array}$ & $\mu \beta / \mathrm{M}_{\mathrm{w}}$ & $B$ \\
\hline & DR1 & 475 & 800 & 3.0 & $5 \times 10^{6}$ \\
\hline & DANS & 438 & 580 & 2.1 & $2 \times 10^{4}$ \\
\hline & TCVDPA & 531 & 584 & 1.7 & $2 \times 10^{7}$ \\
\hline & CLD & 695 & 35000 & 45.7 & N/A \\
\hline & FTC & 650 & 18000 & 25.9 & N/A \\
\hline
\end{tabular}

\subsection{Poling}

Nonlinear optical polymers (NLO) consist of chromophores mixed in a polymer as guest, attached to the polymer (side chain or main chain) or crosslinked between polymer chains. The chromophores have to be aligned to have the desired electro-optical activity. Different chromophore orientation techniques are described in section 2.4. This is done by heating the polymer to just below the glass transition temperature and applying a high DC field (nearly $100 \mathrm{~V} / \mu \mathrm{m}$ ) which aligns the chromophores. The poling process is shown in Fig. 3.8 .

The electro-optic activity in a poled film is characterized by the nonlinear coefficient $r_{33}$. The $\mathrm{r}_{33}$ value of an EO polymer can be expressed as,

$$
r_{33}=\frac{2 N \beta f_{\omega}\left\langle\cos ^{3} \theta\right\rangle}{n^{4}}
$$

where $\mathrm{N}$ is the chromophore number density, $\beta$ is the chromophore first order hyperpolarizability, $\mathrm{n}$ is the index of refraction, $f_{\omega}$ is a local optical field correction factor, $<\cos ^{3} \theta>$ is the order parameter. It is an average over the orientation of all chromophores, with $\theta$ their angle with the electrical field. For perfect alignment $\left\langle\cos ^{3} \theta>=1\right.$, in practice it is $<0.5$. 
In the absence of electrostatic interactions the order parameter can be expressed as,

$<\cos ^{3} \theta>=\mu \mathrm{F} / 5 \mathrm{kT}$

where, $\mu$ is the dipole moment, $F$ the poling field strength, $\mathrm{k}$ the Boltzmann constant and $\mathrm{T}$ is the poling temperature.

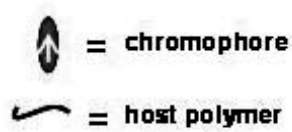

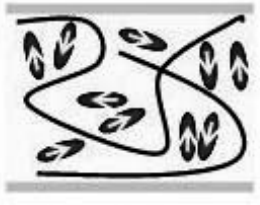

Heat to $\mathrm{Tg}$

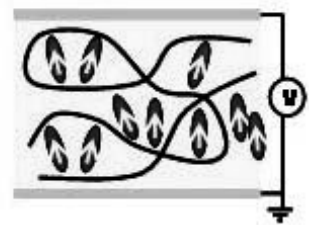

Cool to RT

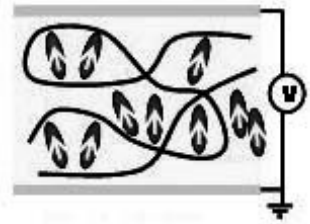

Apply $\vee p$

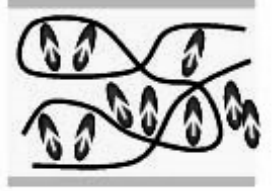

Switch off $\vee p$

Figure 3.9: The poling process.

The different parameters to be optimized during poling are the poling temperature, poling voltage and poling time. The poling voltage is limited by the breakdown voltage of the polymer which is about $100 \mathrm{~V} / \mu \mathrm{m}$ for most of the polymers. Poling is an exponential process strongly dependent on temperature. In our case the poling temperature was chosen just below the $\mathrm{T}_{\mathrm{g}}$ so that most of the poling, about $90 \%$, takes place during the first 10 minutes. For efficient poling a time of 20 minutes was chosen in all the cases. The poling temperature is the most important factor influencing the poling process. At lower than optimum temperature, the free movement of the chromophores is hindered. At temperatures higher than the optimum, thermal agitations will disturb the poling order. The optimum poling temperature is determined by poling the polymer at different temperatures and finding the peak in the plot of $r_{33}$ vs Poling temperature as shown in Fig. 3.10 . 


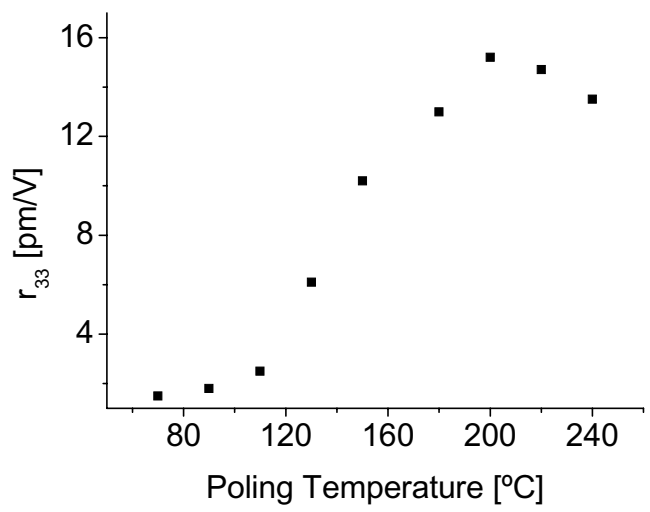

Figure 3.10: Optimizing the poling temperature by poling at different temperatures around the $T_{8}$ of the polymer. The results are obtained with the Polycarbonate-TCVDPA main chain polymer (Chapter 6).

Poling can be monitored by recording the current through the poling electrodes called the poling current. A typical poling curve will be as shown in Fig 3.10a. As the poling voltage is switched $\mathrm{ON}$, chromophores align in an exponential manner in the direction of the electric field. A change in the poling current is also observed during the cooling phase with the poling voltage $\left(\mathrm{V}_{\mathrm{p}}\right)$ ON caused by the resistance change due to the change in temperature.

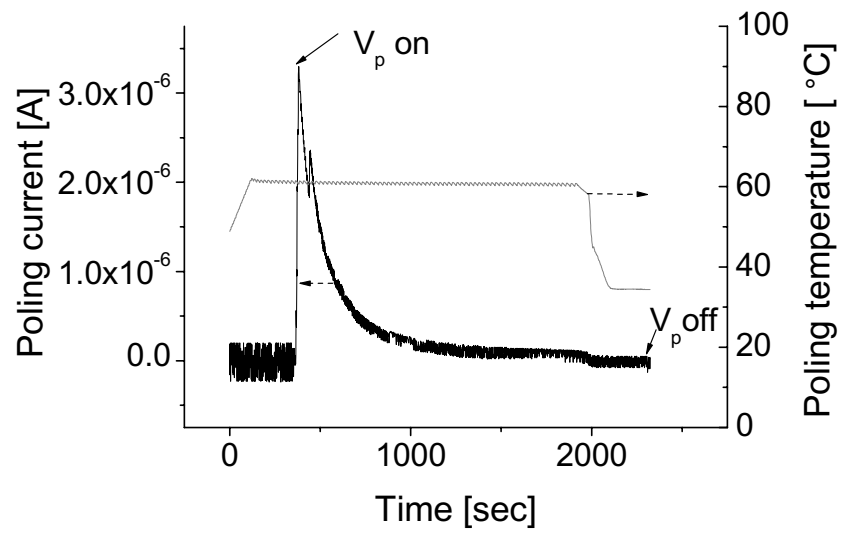

Figure 3.10a: Typical poling behavior showing an exponential decay of the poling current caused by chromophore alignment. The polymer used in this case was SU8 (chapter 5) mixed with 5 wt\% C1. 


\subsection{Experimental setup for the measurement of the $\mathbf{r}_{33}$ coefficient}

Several methods have been proposed to measure the EO coefficient of thin films.

(1) External Mach-Zehnder interferometer: This method requires fabrication of waveguides with suitable cladding layers and hence is time consuming [17].

(2) Fabry Perrot interferometer: The thin film is sandwiched between two semitransparent electrodes and the transmission modulation induced by an electric field enables to determine the EO coefficient [18].

(3) Attenuated total reflection: This consists of detecting the refractive index variation with applied electric field. This method gives accurate results only outside the absorption range $[19,20]$.

(4) Teng-Man ellipsometric method: This method consist of measuring the electric field induced modulation of the interferences between the s-polarized and the p-polarized reflected waves of an EO film sandwiched between a semitransparent and a reflecting electrode. This method is used in the current work because of its simple and fast measuring procedure [21, 22]. This technique does not require the fabrication of waveguides and can also be used in the wavelength regions of the sample where the absorption is high as the light path in the sample has a length of only a few microns.

The Teng-Man setup consists of a polarized laser beam passing through an input polarizer at $45^{\circ}$ so that the light incident on the sample will have equal intensity components of $\mathrm{s}$ and $\mathrm{p}$ polarizations. The beam then passes through a Pockels cell. The relative phase between the orthogonal polarizations can be varied by applying a DC voltage to the Pockels cell. After that the beam enters the sample through a transparent electrode and is reflected by the gold electrode and propagates to the output polarizer which is in the crossed state to the input polarizer. An AC voltage applied to the electrodes induces differential phase shifts to the $\mathrm{s}$ and the $\mathrm{p}$ components of the beam passing through the sample which is converted into amplitude change by the output polarizer and recorded using a detector and a lock-in amplifier.

For the measurement of $r_{33}$ by this technique, the polymer has to be sandwiched between the reflecting gold electrode and the transparent ITO (indium tin oxide) [23] electrode. The experimental setup that has been used is shown in Fig. 3.11. A laser beam is incident at an angle of $45^{\circ}$ on the sample after passing through the input polarizer. The wavelength of the laser can be chosen among $632 \mathrm{~nm}, 830 \mathrm{~nm}, 1300 \mathrm{~nm}$ and $1550 \mathrm{~nm}$. The relative phase between $\mathrm{s}$ and $\mathrm{p}$ components of the laser beam can be varied by varying the voltage applied on the Pockels cell by an amount $\psi_{s p}$. The amount of the relative phase shift for a given voltage on the Pockels cell depends on the wavelength. For the convenience of measurement the sample is placed on a metal frame and the laser beam is reflected onto it using a gold mirror. The reflected beam from the sample is reflected to the detector using another gold mirror as shown in Fig. 3.12.

When the voltage applied to the Pockels cell is changed, the polarization state of the light incident on the sample also changes. By varying the relative phase between $\mathrm{s}$ and $\mathrm{p}$ components from 0 to $2 \pi$, the polarization state of the light incident on the sample can be changed from first a linearly polarized state at $45^{\circ}$ to the incident plane through a circularly polarized state and finally again to a linearly polarized state at $135^{\circ}$ to the 
incident plane. Thus as the voltage on the Pockels cell is varied the amplitude of the signal detected by the detector can be described by (Fig. 3.13a)

$$
I_{o}=2 I_{c} \sin ^{2}\left(\psi_{s p} / 2\right)
$$

where, $I_{c}$ is the half maximum of the detector output. When an AC $V=V_{m} \sin \omega_{m} t$ is applied to the sample, a change in the phase angle $\delta \psi$ in both the $\mathrm{s}$ and $\mathrm{p}$ waves is induced in proportion to the $r_{13}$ and $r_{33}$ coefficients respectively, by the change in refractive index $\delta n$ due to electro-optic effect.

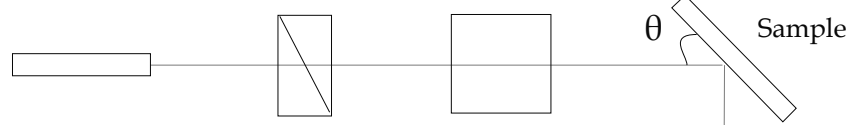

Laser

Polariser Pockels cell
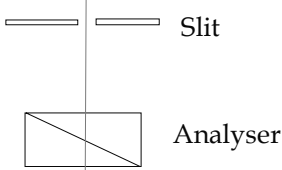

Lens

Detector

Figure 3.11: Experimental setup for the measurement of the $r_{33}$ and $r_{31}$ coefficients by the simple reflection technique of Teng-Man.

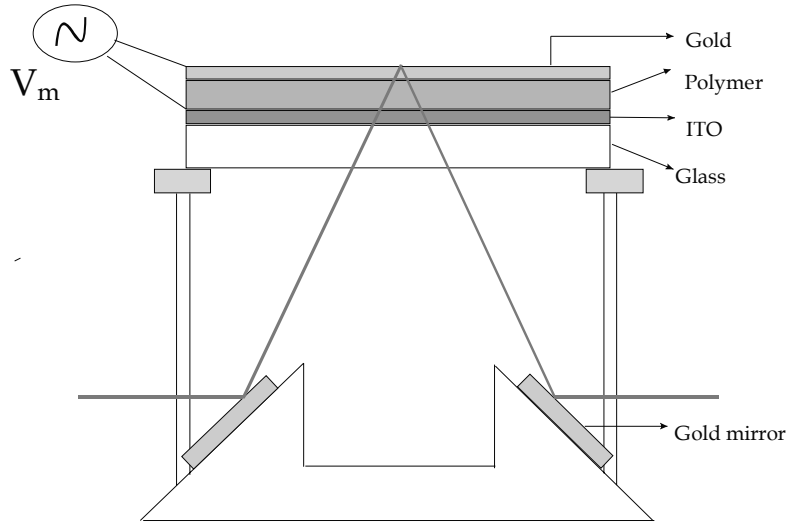

Figure 3.12: A close view of the sample holder used for the $r_{33}$ measurement. 
By choosing the phase retardation such that the output intensity is biased at either A or B (Fig. 3.13) the curve is in its linear region and for small modulations, the ratio between the modulated beam intensity $I$ and the midpoint intensity $I_{c}$ can be approximated by

$$
I / I_{c} \approx \delta \psi_{s p}
$$

The electro optic coefficient $\mathrm{r}_{33}$ is given by [21],

$$
r_{33}=\frac{3 \lambda I_{m}}{4 \pi V_{m} I_{c} n^{2}} \frac{\left(n^{2}-\sin ^{2} \theta\right)^{3 / 2}}{\left(n^{2}-2 \sin ^{2} \theta\right)} \frac{1}{\sin ^{2} \theta}
$$

where, $I_{m}$ is the amplitude of modulation and $\mathrm{n}$ is the refractive index of the polymer film and $\theta$ is the angle of incidence.

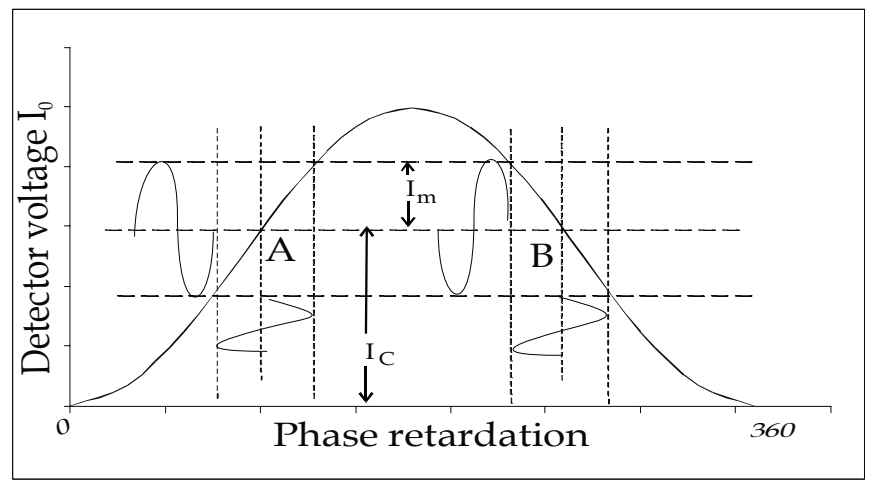

Figure 3.13: Output intensity $I_{0}$ (which is proportional to the output voltage of the detector) as a function of the phase retardation provided by the Pockels cell.

As the voltage on the Pockels cell is varied the output intensity moves along the curve shown in Fig. 3.13 and hence the modulation amplitude $I_{m}$ also changes. A plot of the modulation amplitude $I_{m}$ versus $I_{c}$ will give an ellipse as shown in Fig. 3.14a. The vertical axis of the ellipse gives a measure of the magnitude of the electro-optic coefficient. If no other modulation effect is present except the electro-optic modulation, the magnitude of the modulation amplitude should be the same at A and B. But in reality the signal is affected by interference effects between the light reflected from two sides of the polymer film which makes the ellipse asymmetric about the axis. This interference effect can be eliminated by subtracting the modulation amplitude $\left(I_{m}\right)$ at A and B, as the interference modulation does not change sign when one switches from point $\mathrm{A}$ to $\mathrm{B}$.

The slope of the ellipse which is related to the modulation of the reflectivity varies with the angle of incidence $\theta$. In the wavelength region where the transparency of the electrode 
is less, the reflectivity modulation becomes high. At certain angles of incidence this reflectivity modulation becomes highly enhanced by the Fabry-Perrot resonances of the layer stack. For illustration, Fig. 3.14b shows the ellipse measured at different angles of the incident beam showing different angles of tilt.

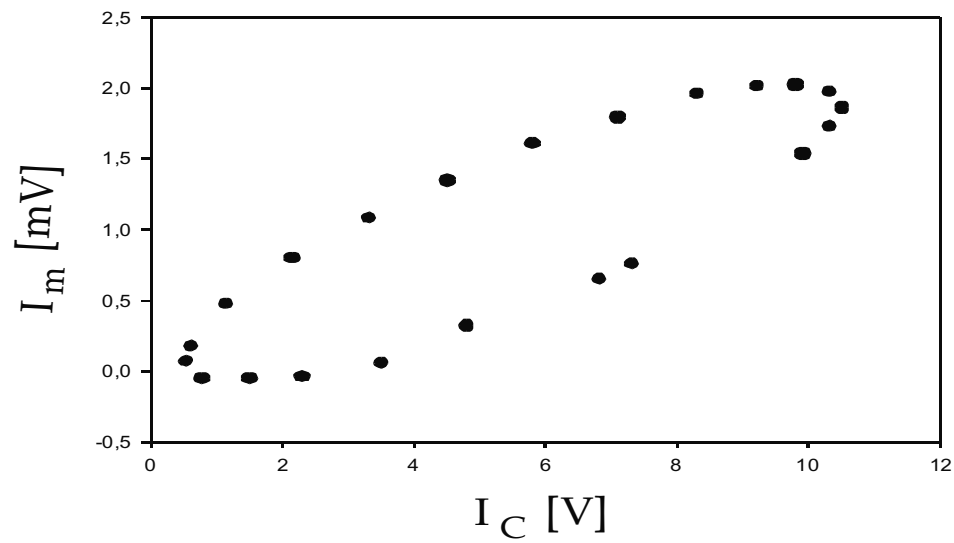

Figure 3.14a: Variation of the optical AC modulation amplitude $I_{m}$ as a function of $I_{C}$ obtained by sweeping the DC light level over the curve in Fig. 3.13, measured at $1300 \mathrm{~nm}$ and showing very high reflectivity modulation.

In the wavelength region above $1 \mu \mathrm{m}$ the transparency of ITO becomes so low that the reflectivity modulation can induce errors as high as 50\%. When measured at $830 \mathrm{~nm}$, measurements can be made with an error of $8 \%$ [24]. When the wavelength is far from the absorption band of ITO [23] the change in the vertical axis of the ellipse due to the reflectivity modulation is small and can be neglected. When ITO is highly absorbing the reflectivity of the polymer/ITO interface becomes high and the reflectivity modulation is highly enhanced by Fabry-Perot resonances and the measurement error becomes large. Fig. 3.14a shows a measurement made at $1300 \mathrm{~nm}$. It can be seen that the ellipse is highly tilted because of reflectivity modulation. Therefore in the region where ITO is absorbing, it has to be replaced by other transparent electrode material. Our measurements have normally been made at $830 \mathrm{~nm}$ where the effects of ITO absorption are small. 

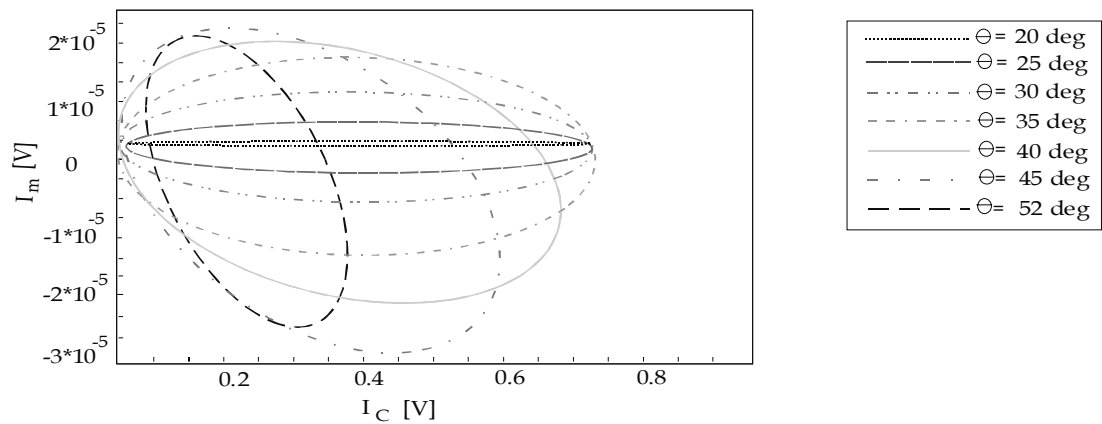

Figure 3.14b: Electro-optic ellipsometric measurement made at different angles of incidence.

Fig. 3.15 shows the measurements made at $830 \mathrm{~nm}$. The ellipse is almost symmetric to the axis. This proves that there is nearly no influence of the reflectivity modulation and the measured value for the ellipse axis is purely electro-optic. There is another contribution to the tilt in the ellipse axis by the pressure exerted on the sample when the electrode needles are pressed against the sample, see Fig. 3.16. But still the error introduced is less than $8 \%$.

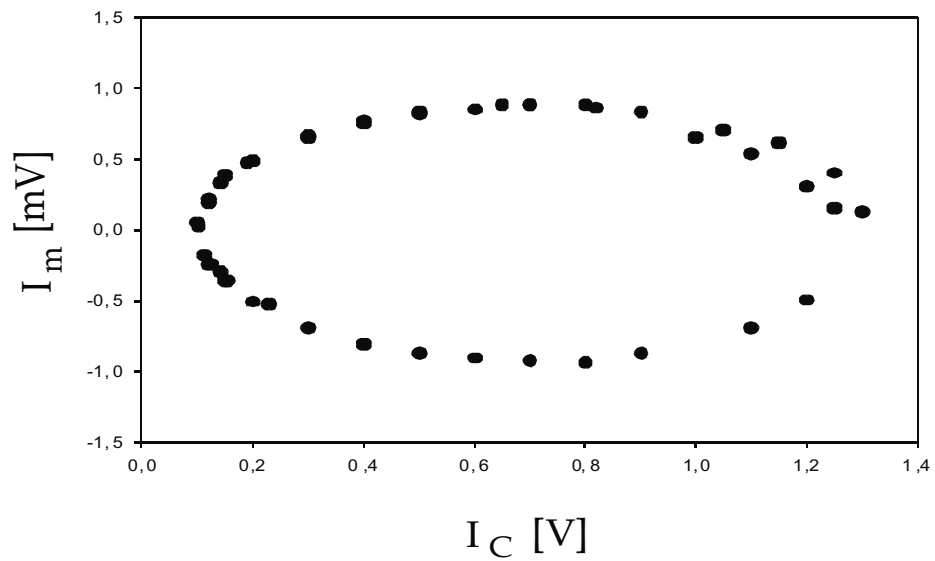

Figure 3.15: Variation of the optical AC modulation amplitude $I_{m}$ as a function of $I_{C}$ obtained by sweeping the DC light level over the curve in Fig. 3.13 measured at $830 \mathrm{~nm}$. 


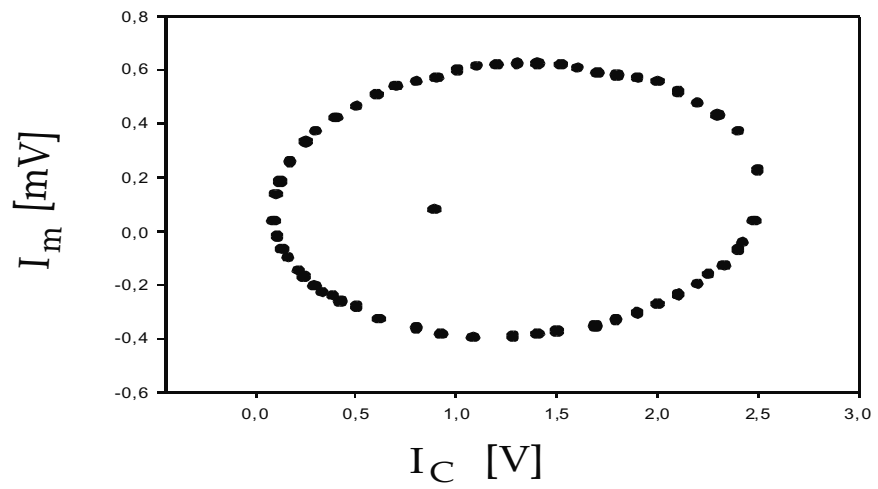

Figure 3.16: Variation of the optical AC modulation amplitude $I_{m}$ as a function of $I_{C}$ obtained by sweeping the DC light level over the curve in Fig. 3.13 measured at $830 \mathrm{~nm}$ with high electrode needle pressure on the sample.

\subsubsection{Sample preparation for measurement of $\mathbf{r}_{33}$}

Glass wafers coated with $30 \mathrm{~nm}$ ITO were cleaned in $\mathrm{HNO}_{3}$ and etched to form the patterned ITO bottom electrode. The polymer was spun on the glass wafer with the patterned ITO layer. It was baked at $95^{\circ} \mathrm{C}$ on a hot plate for 1 hour and in a vacuum oven at $120^{\circ} \mathrm{C}$ for 8 hours. A $100 \mathrm{~nm}$ gold layer was sputtered on it. During sputtering the temperature raises above the glass transition temperature of the polymer causing the gold layer above it to buckle. The buckling of gold was prevented by subdividing the total sputtering time in shorter periods and allowing sufficient cooling time in between.

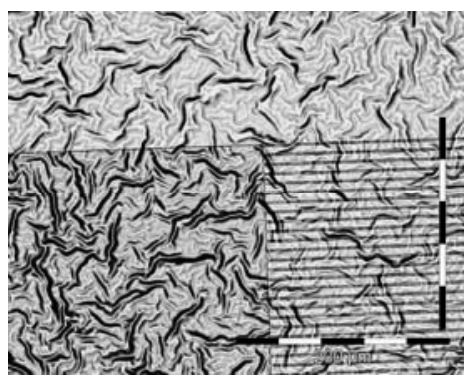

a

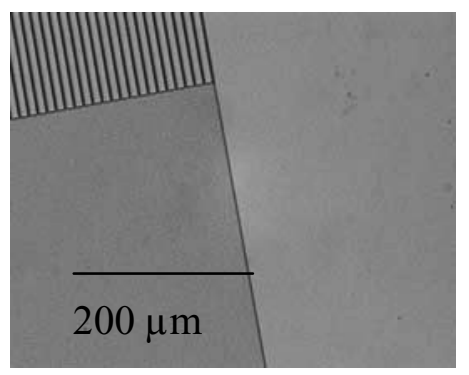

b

Figure 3.17: Microscope picture of the gold layer on SU8; (a) directly sputtered (b) result obtained after optimization of the sputtering process.

Fig. 3.17a shows the buckling phenomena of the gold on top of a polymer and Fig 3.17b shows a smooth gold layer on top of a polymer by controlled sputtering. The patterned 
photoresist layer used for gold etching can be seen on top. The top gold electrode is then patterned by wet chemical etching.

\subsection{In-situ poling with the Teng-Man setup}

The Teng-Man setup was modified by incorporating a heating element so that it can also be used as a poling setup when applying a DC bias voltage. The heating element consists of thin film chromium heaters on a glass wafer made by lift-off technique. The final arrangement of the experimental set-up is shown in Fig. 3.18. A transparent thermally conducting refractive index matching fluid is used in-between the sample and the heating wafer. The temperature of the wafer can be raised up to about $200^{\circ} \mathrm{C}$ by this setup. The temperature is controlled within $1^{\circ} \mathrm{C}$ by using a thermocouple and a feedback circuit. Poling can be monitored by recording $I_{c}$ (birefringence-DC detector current converted to voltage) or $I_{m}$ (The current signal from the detector was pre-amplified which converts it to an voltage and the EO modulation amplitude was recorded with a lock-in amplifier). Recording $I_{c}$ is a more straight forward approach. The later involves monitoring the EO effect by keeping the output at points A or B which constantly shifts during poling because of poling induced birefringence [25].

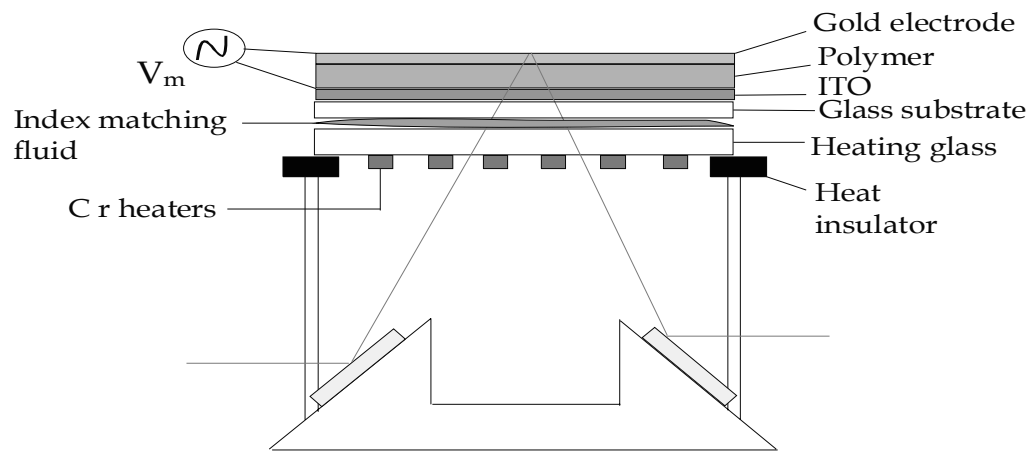

Figure 3.18: Modified Teng-Man set-up with heating element.

\subsubsection{Measuring the poling efficiency by monitoring birefringence}

Poling was monitored by recording Ic. During poling the polymer becomes birefringent which shifts the bias point of the curve in Fig 3.13 and hence induces a change in the DC light level as shown in Fig 3.19.

Poling is done by heating the wafer to a temperature nearby the $\mathrm{T}_{\mathrm{g}}$ of the polymer. The voltage on the Pockels cell is adjusted such that the output is biased at the point A or B of Fig. 3.13. A DC voltage was then applied between the ITO and gold to start the poling 
process. During poling the DC signal from the output polarizer is recorded using a detector and a multimeter. As poling proceeds, the film becomes birefringent inducing a phase shift between the $\mathrm{s}$ and the $\mathrm{p}$ polarizations which shifts the bias point resulting in a variation of the output amplitude. Fig. 3.19 shows the change in the output detector voltage recorded during poling.

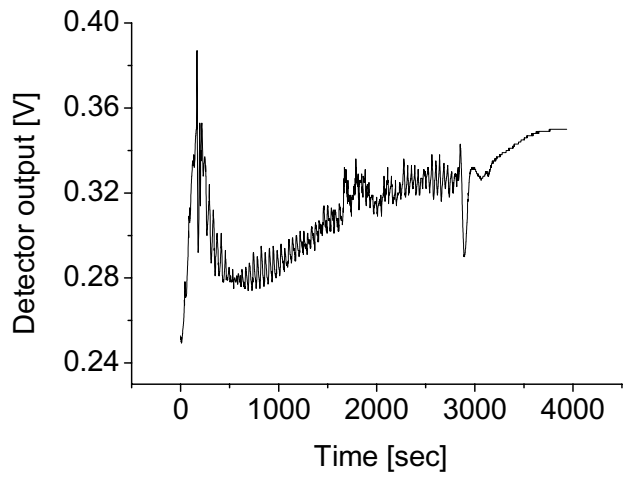

Figure 3.19: In-situ poling by monitoring birefringence.

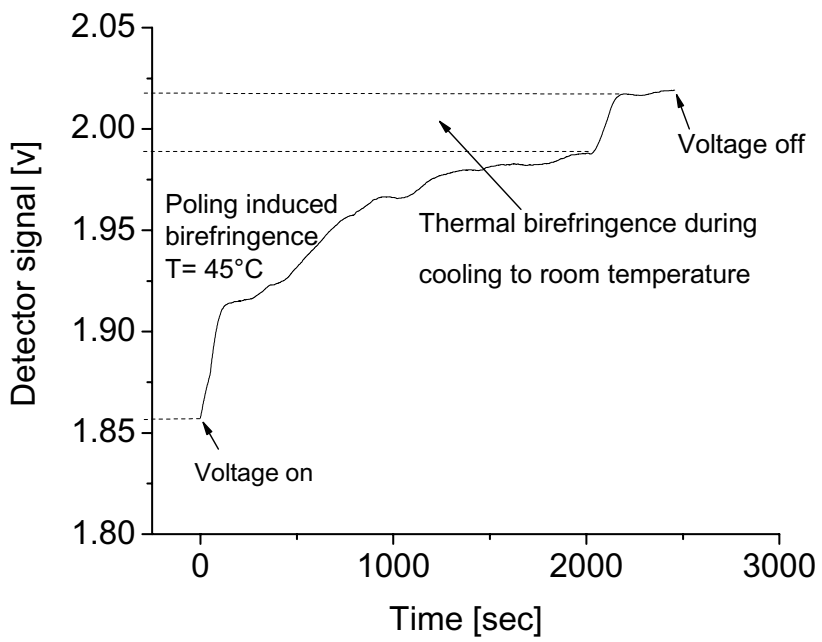

Figure 3.20: Thermal and poling induced birefringence.

Fig. 3.20 shows the thermal and poling induced birefringence monitored during in-situ poling. Figs. 3.19 and 3.20 essentially show similar in-situ poling experiments on different 
samples. Thermal birefringence is a consequence of the induced stress by thermal expansion of the sample by heating the sample to $T_{g}$ and during cooling. When the temperature remains constant, the change in $I_{C}$ is caused only by poling induced birefringence.

\subsubsection{Monitoring EO modulation amplitude}

Poling can also be monitored recording the ac modulation amplitude during poling. This is done by a lock-in technique. Fig. 3.21 shows in-situ poling done by observing the ac modulation amplitude. The sections 1 and 3 showing rapid increase and decrease in the ac modulation amplitude are obtained during heating and cooling of the sample to $\mathrm{T}_{\mathrm{g}}$ and back to room temperature. They are a consequence of thermally induced stress. Section 2 of the curve shows the poling process at constant poling temperature. The bias point $\mathrm{A}$ or $\mathrm{B}$ was chosen such that the EO modulation has an opposite sign to that of the stress induced modulation which has the same sign at both the bias points. Under these conditions a decrease in the modulation amplitude can be seen (section 2 of Fig. 3.21) when the poling proceeds.

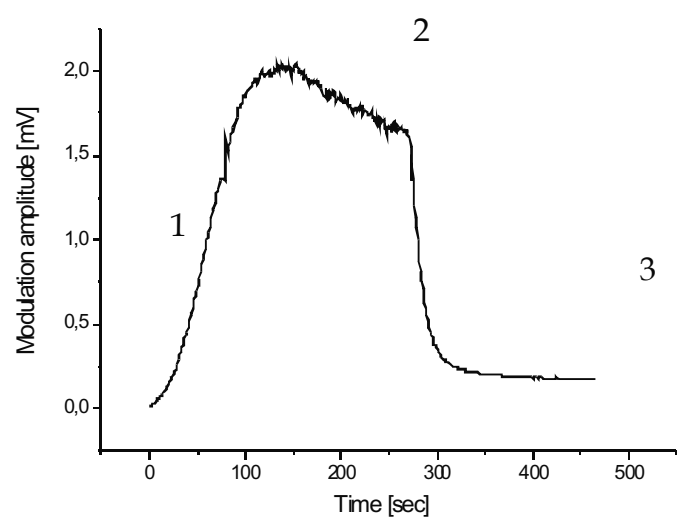

Figure 3.21: In-situ poling by monitoring the EO modulation amplitude. Section 1 and 3 are taken during heating and cooling respectively and section 2 is during poling.

Fig. 3.22 demonstrates more clearly the stress induced modulation. The sample was heated to its $\mathrm{T}_{\mathrm{g}}$ and an $\mathrm{AC}$ ripple was applied. Fig 3.22a shows the stress induced modulation at twice the frequency of the driving signal when the $\mathrm{AC}$ ripple is biased at 0 $\mathrm{V}$ as the polymer film responds in the same way to both the positive and negative voltage cycles. Figures 3.22b-3.22d show the change in the stress induced modulation as a DC bias is applied to the AC ripple. Now as the component of the voltage during the negative cycle reduces due to the applied DC bias, also one component of the optical modulation reduces progressively to zero as the negative cycle of the AC ripple becomes zero. This effect was observed only close to the $\mathrm{T}_{\mathrm{g}}$ of the sample when the polymer is in its rubbery 
state. The huge change in the electro-optic modulation amplitude observed during the heating and cooling cycles in Fig 3.21 is caused by this effect.

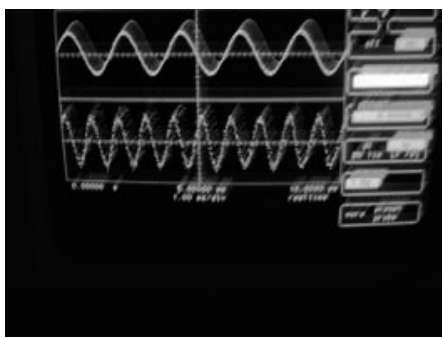

a

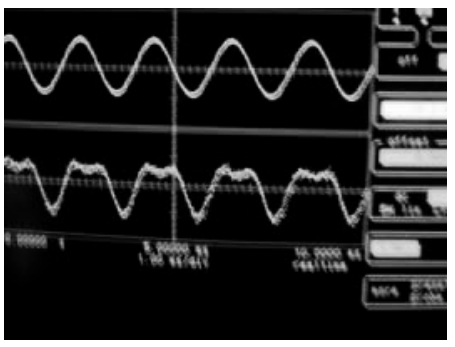

C

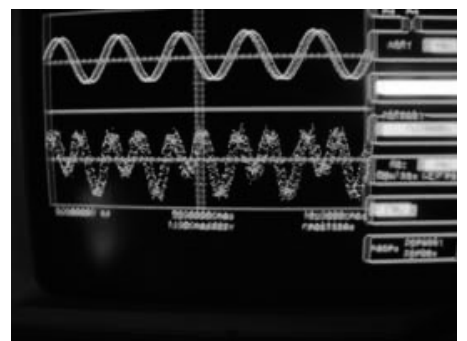

$\mathrm{b}$

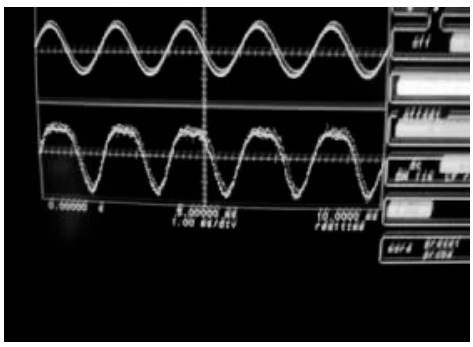

d

Figure 3.22: Thermally induced stress modulation as the poling DC field is increased from zero to $V p$. The top sine wave is the electrical signal and the bottom curve is the optical response in all the pictures from a to $d$. $a$ : DC bias is zero, $b$ and $c$ : DC bias is increased, $d: D C$ bias is almost equal to the amplitude of the AC ripple.

\subsection{Enhancement of the poling efficiency}

In the fabrication of practical EO devices the maximum achievable $\mathrm{r}_{33}$ coefficient should be optimized. One of the major problems encountered in optimizing polymeric EO materials is to translate the large $\beta$ values of organic chromophores into large macroscopic EO activity. According to the ideal gas model, macroscopic optical nonlinearity should scale as $\mu \beta / M_{w}\left(M_{w}\right.$ is the molecular mass of the chromophore) [26]. Therefore EO coefficients of many hundreds of pm/V should be expected if such optical nonlinearity of the chromophores could be translated into macroscopic EO activity. However the electric field poling of a polymer containing highly nonlinear chromophore is hindered by the large dipole moment of the molecules. Although the dipole moment aids in aligning the 
chromophore, it also leads to dipole-dipole interactions between the chromophores resulting in anti-parallel clustering of the chromophores during poling and reducing maximum achievable order parameter. Theoretical analysis suggests that the maximum realizable EO activity can be enhanced by modifying chromophores with bulky substituent groups [27]. These groups do not influence the molecular hyperpolarizability but will minimize the effect of unwanted electrostatic interactions [28-33] by hindering the chromophores approaching each others side by side. Theoretical analysis also suggests that the ideal chromophore shape is that of a sphere.

The substituents have two effects: one is that the different shape and size of substituents will lead to different intermolecular electrostatic interactions between the molecules (preventing closer approach of the chromophores); the other is that the different rigidity and size of the substituents will also create variability of free space (site isolation effect) which affects the mobility of the chromophores under high electric field poling conditions.

TCVDPA chromophores with different bulky groups ( $\mathrm{C} 1$ to $\mathrm{C} 6$ as described above, section 3.2.3) were synthesized. In order to study the substituent effect on the poling efficiency, the different chromophores C1-C6 were mixed to Polysulfone at different concentrations and the resulting polymeric material was processed, poled and $\mathrm{r}_{33}$ was measured. Bulkiness of the chromophore increases the poling efficiency, but at the same time with increasingly bulky substituents, the achievable effective concentration $\left(w^{0} \%_{\text {eff }}\right)$ is reduced. The effective concentration of the chromophore is given by:

$$
w^{0} \%_{\text {eff }}=w^{0} \% * \frac{M_{\text {bare }}}{M_{\text {bulky }}}
$$

where, $w \%$ is the weight percent concentration of the chromophore with the bulky group and $M_{\text {bare }}$ and $M_{\text {bulky }}$ are the molecular masses of the bare and the bulky chromophores respectively.

Samples were prepared as explained in section 3.4.1. The films were contact-poled at the respective $T_{g}$ for $20 \mathrm{~min}$ with DC electric field of $100 \mathrm{~V} / \mu \mathrm{m}$. The $\mathrm{r}_{33}$ values were measured using the Teng-Man reflection technique at a wavelength of $830 \mathrm{~nm}$.

The chromophore loading could be varied over a wide range without phase separation and the resulting materials could easily be processed into films with high optical quality. As shown in Fig. 3.23, for unmodified chromophore C1, the EO activity increases linearly with loading up to $15 \mathrm{wt} \%$, after which the curve starts to deviate from linearity, showing evidence of chromophore-chromophore electrostatic interactions. A similar behavior was noticed for chromophore $\mathrm{C} 2$, in which the methoxy groups are not large enough to significantly affect the interactions, but only providing a small improvement of about 2 $\mathrm{pm} / \mathrm{V}$ with respect to $\mathrm{C} 1$. Moreover, $\mathrm{C} 1$ and $\mathrm{C} 2$ remain at lower $\mathrm{r}_{33}$ values compared with other bulky substituted chromophores, like C3, C4 and C5 suggesting a significantly higher dipole-dipole interaction already at low loadings. However, the increment in 46 
poling efficiency provided by the tert-butyl groups of $\mathrm{C} 3$ and the branched alkoxy moieties of $\mathrm{C} 4$ is clearly visible already at low chromophore loadings, whereas at larger concentrations the shape modification is even more effective showing a nearly doubling of $r_{33}$ at $35 w_{\text {eff }}$ with respect to $\mathrm{C} 1$ (from $12.5 \mathrm{pm} / \mathrm{V}$ to $25 \mathrm{pm} / \mathrm{V}$ ). The best result was obtained with chromophore $\mathrm{C} 5$ that provides a more significant enhancement of the EO response reaching its maximum $\mathrm{r}_{33}$ of $25 \mathrm{pm} / \mathrm{V}$ at already $30 w \%_{\text {eff. Probably, the very }}$ bulky pentafluorobenzyl groups provide the site isolation needed for free chromophore reorientation under electric field poling conditions. In the case of the dendritic chromophore C6, an EO coefficient of only $5.5 \mathrm{pm} / \mathrm{V}$ was obtained at $10 w \%_{\text {eff. }}$ Even though this chromophore is the bulkiest of the whole series and thus it is expected to induce the highest poling efficiency, its $\mathrm{r}_{33}$ value recorded at $10 w \%$ eff does not even follow the linear slope observed for the smaller fluorinated aromatic derivative $\mathrm{C} 5$. This could possible be because of change in the $\beta$ value of the chromophore as shown in Table 3.2. This was also evident from the pale purple color of the film made with C6 chromophore while the films made from other chromophores were deep purple in color. This could be an indication of a shift in the $\lambda_{\max }$ of the chromophore. These results suggest that a good compromise has to be found for a given chromophore in order to achieve the site isolation needed for maximizing the poling efficiency keeping at the same time a possible minimum value of the chromophore molecular mass.

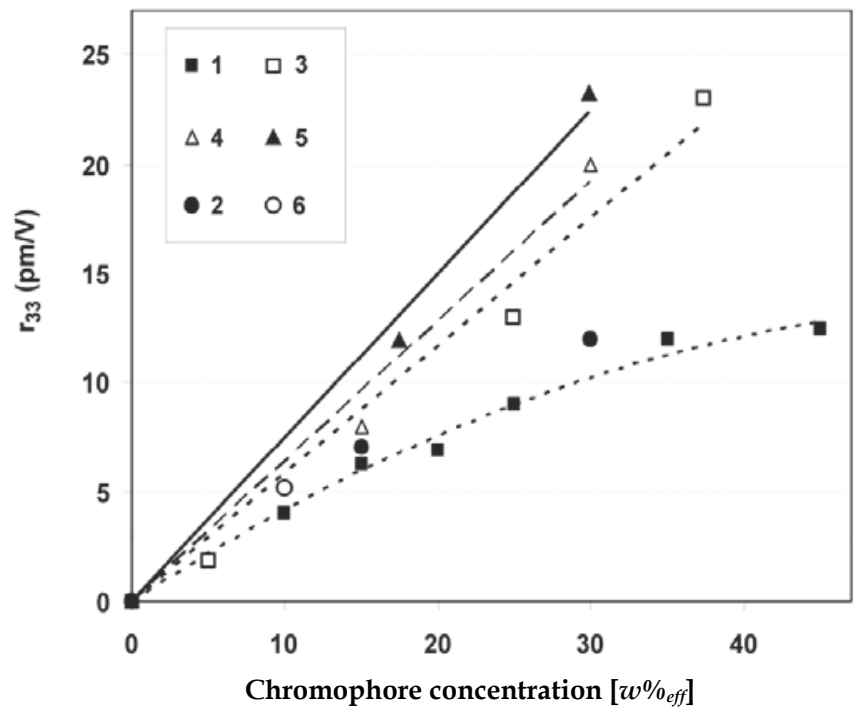

Figure 3.23: Measured values of $r_{33}$ for TCVDPA and its modifications.

Looking for chromophores C1, C3 and C5 in Fig. 3.23 three different trends in the slope of the $r_{33}$ curves can be observed. As in the linear regions of the curve there can be no intermolecular interactions, the different slopes of the curves are because of the site isolation effect as the $\mu \beta$ values of all these chromophores are also the same. It can be 
concluded that, since the hyperpolarizability is similar for all chromophores, the enhancements in $r_{33}$ for the shape modified chromophores are not due to improvements at the molecular level, but indeed to reduced electrostatic interactions between chromophores and hence to a better poling efficiency.

\subsection{Effect of chromophore concentration on $T_{g}$}

Chromophores act as plasticizers and reduce the $T_{g}$ of the host polymer. This is a common behavior in guest-host systems. The size of the chromophore has an influence on the magnitude of the plasticizing effect with smaller molecules having a stronger effect. This is an important factor limiting the maximum achievable chromophore concentration in a guest-host polymer before $T_{g}$ reduces below allowable limits.

For comparison, $\mathrm{T}_{\mathrm{g}}$ has been measured for different chromophore loading densities for films made with chromophores C1, C3, and C6 in Polysulfone. From the curves shown in Fig. 3.24, a clear decay of the $\mathrm{T}_{\mathrm{g}}$ of the material can be seen upon increasing the chromophore concentration. An interesting trend can be noticed in the decay: the bulkier the chromophore $(\mathrm{C} 1<\mathrm{C} 3<\mathrm{C} 6)$, the lower its plasticization effect is. In fact, when $\mathrm{C} 1$ is incorporated in PS at $45 \mathrm{wt} \%$, the $\mathrm{T}_{\mathrm{g}}$ of the resulting blend drops to $80^{\circ} \mathrm{C}$. Such a low $\mathrm{T}_{\mathrm{g}}$ is detrimental for the long term efficiency of a device, causing serious relaxation of the molecular alignment. Differently, dendritic chromophore C6 at $50 \mathrm{wt} \%$ loading displays a much improved $\mathrm{T}_{\mathrm{g}}$ of $130^{\circ} \mathrm{C}$, resulting in a better material for long term device applications.

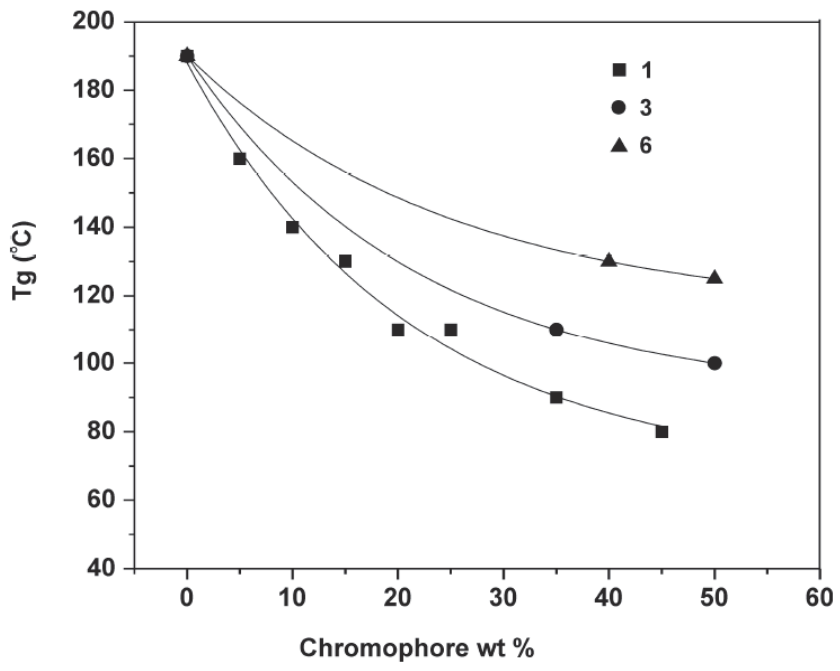

Figure 3.24: Effect of chromophore concentration on $T_{8}$ for chromophores $C 1, C 3$ and C6. 


\subsection{Chromophore relaxation measurements}

As can be seen from the previous section, the chromophore concentration has a strong influence in reducing the $\mathrm{T}_{\mathrm{g}}$. This in turn can have serious effect on chromophore relaxation after poling during device fabrication and operation.

Monitoring the relaxation of the chromophores after poling is shown in Fig 3.24 as function of temperature at constant time. After poling the PS-TCVDPA system did not show any considerable relaxation behavior at room temperature. The temperature was then increased from $30^{\circ} \mathrm{C}$ to $80^{\circ} \mathrm{C}$ in steps of $10^{\circ} \mathrm{C}$ and the poled sample was held at each temperature step for 1 hour. The EO coefficient was measured after each temperature step.

Fig. 3.24 shows the relaxation behavior of $\mathrm{C} 2$ and $\mathrm{C} 5$ chromophores. The concentrations of these two chromophores were chosen carefully in such a way that the $\mathrm{T}_{\mathrm{g}}$ of these polymer are the same, about $120^{\circ} \mathrm{C}$. Therefore the difference in the relaxation behavior is only because of the choromophore size effect in this case. The chromophore relaxation starts even at $50^{\circ} \mathrm{C}$ in both cases, the effect being larger in the case of C5. Because of the different shapes of the chromophores and different free volumes around them, the relaxation dynamics differs in each case.

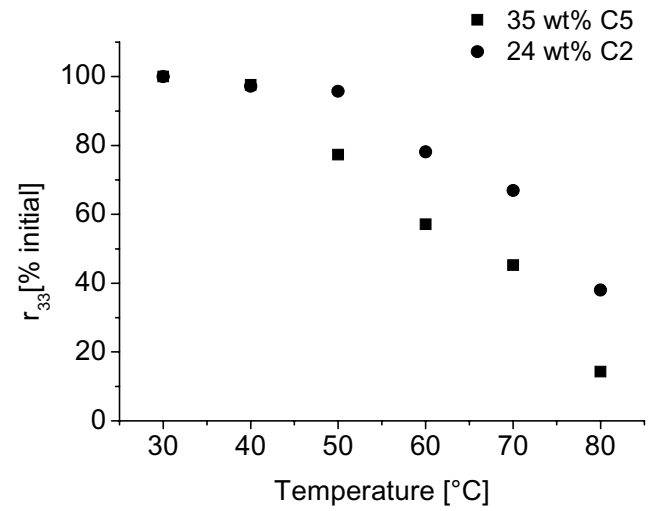

Figure 3.24: Relaxation of TCVDPA chromophores C2 and C5 in PS as a function of temperature showing the effect of chromophore size on relaxation. In both cases $T_{g}$ was $120^{\circ} \mathrm{C}$.

In Fig. 3.25 two different $\mathrm{C} 2$ concentrations are compared. The difference in relaxation in this case is purely because of the difference in $T_{g}$, with clearly the low $T_{g}$ polymer showing fast relaxation. 


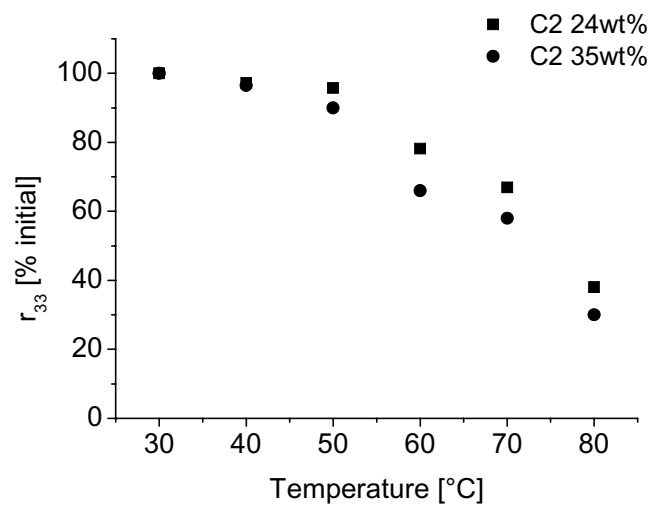

Figure 3.25: Relaxation of TCVDPA chromophore C2 incorporated with two different concentrations of $24 w t \%$ and $35 w t \%$ in PS with $T_{8} s$ of $120^{\circ} \mathrm{C}$ and $100{ }^{\circ} \mathrm{C}$, showing difference in relaxation behavior purely because of difference in $T_{g}$.

In Fig. 3.26 relaxation behaviors of $\mathrm{C} 2$ and $\mathrm{C} 5$ chromophores with same concentrations of $35 \mathrm{wt} \%$ from the Figs. 3.24 and 3.25 are repotted together showing the combined effect of the chromophore size and $\mathrm{T}_{\mathrm{g}}$ on the relaxation process.

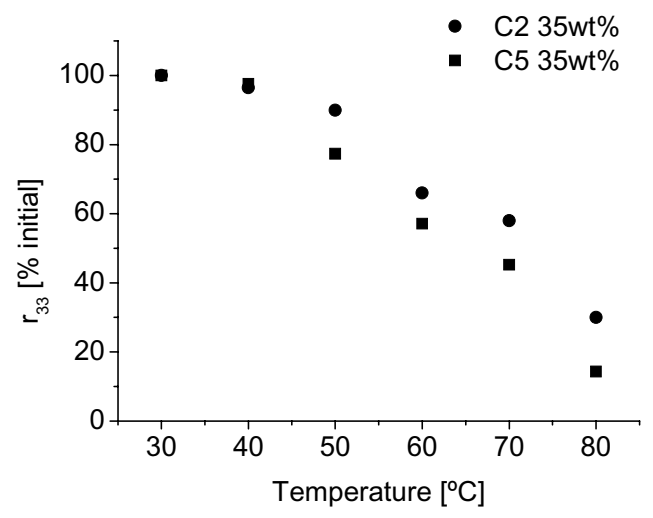

Figure 3.26: Relaxation TCVDPA chromophores C2 and C5 at constant temperature, $T_{g}$ being $100^{\circ} \mathrm{C}$ for $\mathrm{C} 2$ and $120^{\circ} \mathrm{C}$ for $\mathrm{C} 5$.

The relaxation behavior at constant temperature as a function of time is shown in Fig.3.27. The $\mathrm{T}_{\mathrm{g}}$ of the polymers with $\mathrm{C} 2$ and $\mathrm{C} 5$ were $110^{\circ} \mathrm{C}$ and $130^{\circ} \mathrm{C}$ respectively. The relaxation time constants were 645 mins and 720 mins for C2 and C5 respectively. After about 5000 
mins the relaxation process stabilizes with no further noticeable reduction in $r_{33}$. After stabilization the reduction in $\mathrm{r}_{33}$ was found to be $33 \%$ and $14 \%$ for $\mathrm{C} 2$ and $\mathrm{C} 5$ respectively.

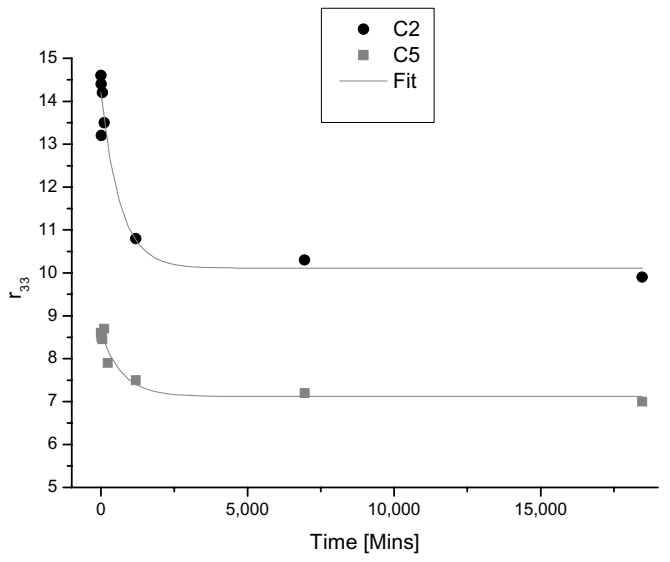

Figure 3.27: Relaxation TCVDPA chromphores $C 2$ and $C 5$ at $50^{\circ} \mathrm{C}$ as a function of time for different $T_{g}$.

It can be concluded from the above discussion that although the chromophore shape has an influence on the relaxation process, the $T_{\mathrm{g}}$ of the material plays the essential role in the relaxation process.

\subsection{Conclusions}

The TCVDPA chromophore was modified with different bulky side groups (C1-C6). The effect of these side groups in reducing the intermolecular interactions during poling is studied by incorporating these chromophores in Polysulfone as guest-host system. Different bulky groups were found to exhibit varying degree of influence in reducing the intermolecular interactions. Modifying the chromophore with bulky groups did not seem to have effect on the hyperpolarizability of the molecule. The bare chromophore $\mathrm{C} 1$ shows a linear increase in $\mathrm{r}_{33}$ upto about $15 \mathrm{w} \%$ after which the intermolecular interaction starts which causes a flattening of the $\mathrm{r}_{33}$ curve. The C3 chromophore with tert-butly groups shows a linear increase in $\mathrm{r}_{33}$ upto $35 \mathrm{w}_{\text {eff }} \%$. A maximum $\mathrm{r}_{33}$ of $24 \mathrm{pm} / \mathrm{V}$ was measured for this chromophore at $35 \mathrm{w}_{\text {eff }} \%$ which is about twice as high as the measured value for $\mathrm{C} 1$. The C5 chromophore which is more bulkier than C3 also showed a similar effect as C3 but with an increased slope of the $r_{33}$ curve. As there cannot be any intermolecular interactions present in the linear regime of the $r_{33}$ curve this difference in the slope could be because of the site isolation effect. The maximum value of $r_{33}$ which was measured with the C5 chromophore was $24 \mathrm{pm} / \mathrm{V}$ at $30 \mathrm{w}_{\text {eff }} \%$. The chromophore C6 which was the bulkiest in the whole series gave a maximum $\mathrm{r}_{33}$ of only $5 \mathrm{pm} / \mathrm{V}$ at $10 \mathrm{w}_{\text {eff }} \%$. It was not possible to incorporate higher concentration of this chromophore because of solubility problems and 
segregation effects. The C5 chromophore was found to have the optimum bulkiness for high poling efficiency and also at the same time enabling incorporation of high concentration. All the chromophores were found to have a plasticizing effect on the host polymer reducing its $\mathrm{T}_{\mathrm{g}}$. The smaller the chromophore size the higher is this effect. The C5 chromophore is also advantageous in this respect that the plasticizing effect is less compared to C3. Photostability measurements show TCVDPA and its modifications are more stable than other known chromophores like DR1. For $20 \mathrm{w} \% \mathrm{C} 1$ in polysulfones slab waveguiding losses were measured to be $1.7 \mathrm{~dB} / \mathrm{cm}$ at $1523 \mathrm{~nm}$ and $6 \mathrm{~dB} / \mathrm{cm}$ at $850 \mathrm{~nm}$. Both the $T_{g}$ and the chromophore size is found to have an effect on the relaxation behavior after the poling field is removed.

\subsection{References}

[1] L. R. Dalton, Adv. Polym. Sci. 1, p158, 2002.

[2] L. R. Dalton, A. W. Harper, R. Ghosn, W. H. Steier, M. Ziari, H. Fetterman, Y. Shi, R. V. Mustacich, A. K. Y. Jen, K. J. Shea, Chem. Mater., 7, p1060, 1995.

[3] T. Verbiest, S. Houbrechts, M. Kauranen, K. Clays, A. Persoons, J. Mater. Chem., 7, p2175, 1997.

[4] S. R. Marder, J. W. Perry, Science, 263, p1706, 1994.

[5] B. Bartosz, H. Yu-Chueh, T. Hidehisa, J. S. Byoung, L. Jingdong ,K. Y. J. Alex, H. William, W. H. Steier, H. R. Fetterman, IEEE J. of selected topics in quantum elect., 13, p1, 2007.

[6] S. Suresh, H. Zengin, B. K. Spraul, T. Sassa, T. Wada, J. D. W. Smith, Tetrahedron Lett., 46, p3913, 2005.

[7] B. K. Spraul, S. Suresh, T. Sassa, M. Angeles Herranz, L. Echegoyen, T. Wada, D. Perahia,D.W. Smith, Tetrahedron Lett., 45, p3253, 2004.

[8] Y. J. Cheng, J. Luo, S. Hau, D. H. Bale, T. D. Kim, Z. Shi, D. B. Lao, N. M. Tucker, Chem. Mater., 19, p1154, 2007.

[9] A. Galvan-Gonzalez, M. Canva, G. I. Stegeman, R. Twieg, T. C. Kowalczyk, H. S. Lackritz, Opt. Lett., 24, p1741, 1999.

[10] R. S. Moshrefzadeh, D. K. Misemer, M. D. Radcliffe, C. V. Francis, S. K. Mohapatra, Appl. Phys. Lett., 62, p16, 1993.

[11] M. A. Mortazavi, H. N. Yoon, C. C. Teng, J. Appl. Phys., 74, p4871, 1993.

[12] A. Galvan - Gonzalez, M. Canva, G. I. Stegeman, R. Twieg, K. P. Chan, T. C. 
Kowalczyk, X. Q. Zhang, H. S. Lackritz, S. Marder, S. Thayumanavan, Opt. Lett., 25, p332, 2000.

[13] M. E. DeRosa, M. He, J. S. Cites, S. M. Garner, Y. R Tang,. J. Phys. Chem. B, 108, p8725, 2004.

[14] R. Dekker, Ph.D. Thesis "All optical processes in integrated optical devices using materials With large third order nonlinearities and gain", University of Twente, Enschede, ISBN- 10:909021436-4, 2006.

[15] A. Galvan-Gonzalez, M. Canva, G. I. Stegeman, L. Sukhomlinova, R. J. Twieg, K. P. Chan, T. C. Kowalczyk, H. S. Lackritz, J. Opt. Soc. Am. B, 17, p1992, 2000.

[16] A. Galvan-Gonzalez., M. Canva, G. I. Stegeman, R. Twieg, K. P. Chan, T. C. Kowalczyk, X. Q. Zhang, H. S. Lackritz, S. R. Marder, S. Thayumanavan, Opt. Lett., 25, p332, 2000.

[17] K. D. Singer, Y. K. Kuzyk, W. R. Holland, S. Lallama, Appl. Phys. Lett., 53, p1800, 1988.

[18] H. Uchiki and T. Kobayashi, J. of Appl. Phys., 64, p2625, 1988.

[19] Y. Levy, V. Dentan, M. Dumont, P. Robin, E. Chastaing, J. Messier, F. Kajzar, P. Prasad, D. Ulrich, NATO ASI Series E., 162, p337, 1989.

[20] M. Dumont, Y. Levy, D. Morichere, J. Messier, F. Kajzar, P. Prasad, NATO ASI Series E., 94, p461, 1991.

[21] D. Haas, H. Yoon, H. T. Man, G. Cross, S. Mann, N. Parsons, Proc. SPIE, 1147, p222, 1989.

[22] C. C. Teng, H.T. Man, Appl. Phys. Lett., 56, p1734, 1990.

[23] C. Coutal, A. Azema, J. C. Roustan, Thin solid films, 288, p248, 1996.

[24] F. Michelotti, G. Nicolao, F. Tesi, M. Bertolotti, Chemical physics 245, p311, 1999.

[25] Horowitz, M. B. Pereira, P. Alcantara, H. P. Grueineisen, V. Stefani, J. of Noncrystalline solids, 284, p183, 2001.

[26] C. R. Moylan, R. D. Miller, R. J. Twieg, S. Ermer, S. M. Loveyjoy, D. S. Leung, Proc. SPIE, 2527, p150, 1995.

[27] A. W. Harper, S. Sun, L. R. Dalton, S. M. Garner, A. Chen, S. Kalluri, W. H. Steier, B. H. Robinson, Opt. Soc. Am., B15, p329, 1998. 
[28] L. R. Dalton, W. H. Steier, B. H. Robinson, C. Zhang, A. Ren, S. Garner, A. Chen, T. Londergan, L. Irwin, B. Carlson, L. Fifield, G. Phelan, C. Kincaid, J. Amend, A. Jen, J. Mater. Chem., 9, p1905, 1999.

[29] A. W. Harper, S. Sun, L. R. Dalton, S. M. Garner, A. Chen, S. Kalluri, W. H. Steier, B. H. Robinson, J. Opt. Soc. Am. B, 15, p329, 1998.

[30] I. Liakatas, C. Cai, M. Bösch, M. Jäger, C. Bosshard, P. Günter, C. Zhang, L. R. Dalton, Appl. Phys. Lett., 76, p1368, 2000.

[31] L. R. Dalton, A. W. Harper, B. H. Robinson, Proc. Natl. Acad. Sci. U. S. A., 94, p4842, 1997.

[32] R. D. Nielsen, H. L. Rommel, B. H. Robinson, J. Phys. Chem. B, 108, p8659, 2004.

[33] B. H. Robinson, L. R. Dalton, J. Phys. Chem. A, 104, p4785, 2000. 


\section{Design and fabrication of laterally coupled PMMA-DR1 microring resonators}

In this chapter the design and fabrication of Laterally coupled microring resonator made of PMMA-DR1 polymer are presented. Special attention is given to the fabrication methods to realize the sub micron gap between the resonator and straight port waveguides. The resulting geometry largely determines the direction of the hybrid approach, which has to be followed. A racetrack design is made to increase the coupling at the minimum possible gap size and poling of these devices is demonstrated.

Published as: M. Balakrishnan et al, SPIE, vol 6123, 612301, 2006. 


\subsection{Introduction}

Microring resonators are gaining increasing importance in high speed applications like switches, modulators [1-4] and filters [5]. Controlling the gap between the ring and the straight waveguide is of great importance for the correct functioning of the device. In the case of vertically coupled microrings the gap can be precisely controlled and can be made to be very small by controlling the thickness of the intermediate layer between the ring and the straight waveguide. But in this case a critical alignment step is required to align the ring to the straight waveguide in order to obtain the desired coupling. The fabrication of the sub-micron gaps becomes a technological difficulty especially in the case of polymers. Although very small gaps of about $50 \mathrm{~nm}$ are possible by using expensive electron beam lithography [6], and nano-imprinting [7], we present here more conventional methods that exploit the maximum possible limits of photolithography.

\subsubsection{The working principle of the microring resonator}

The working principle of a microring resonator is shown in Fig. 4.1.

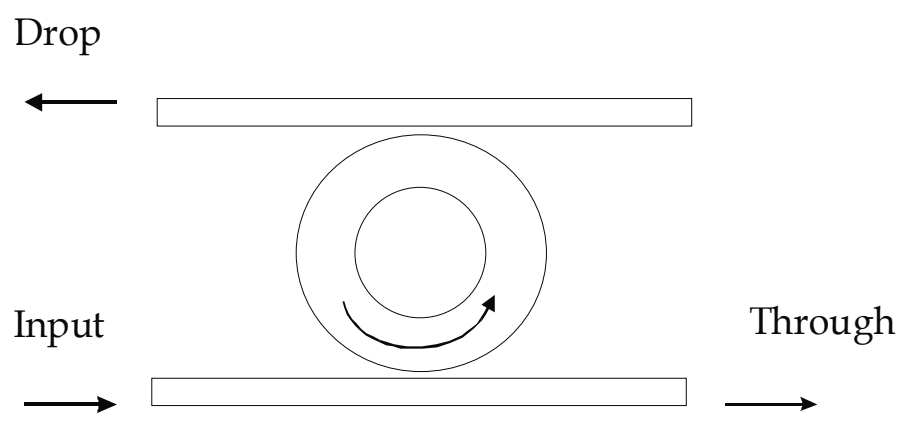

Figure 4.1: Schematic of a microring resonator.

The resonator consists of a ring centered between two waveguides. One waveguide is used as an input and the other as the output port or drop port. The straight waveguides are positioned in such a way that there is a small gap between the ring and the straight waveguide. Because of this gap a fraction of the light of the input port is coupled to the ring with a coupling coefficient $\mathrm{K}_{1}$ [8]. This coupling section is known as a directional coupler $[9,10]$. The light that enters the ring makes half a round trip and a part of the power in the ring couples to the drop port and the second coupling section with coupling constant $\kappa_{2}$. The light then completes the roundtrip in the ring and interferes with the light which has just coupled to the ring from the input waveguide. If the phases of the light in the ring and that of the light entering the ring are equal, constructive interference occurs and there will be build-up of power in the resonator. This happens only when the light in the ring experiences a phase shift of integer times 2 л. In this so-called resonance state it is possible that all the power at a certain wavelength is extracted from the input waveguide 
and directed to the drop waveguide. Otherwise destructive interference will occur and the ring will hardly extract any light.

In Fig. 4.2 the wavelength response of the through and drop port of a microring resonator is schematically given where $\Delta \lambda_{\mathrm{FWHM}}$ is the full width at half maximum of the resonance peak, $\lambda_{1}$ and $\lambda_{2}$ are neighboring resonance wavelengths, and FSR is the free spectral range of the microring.

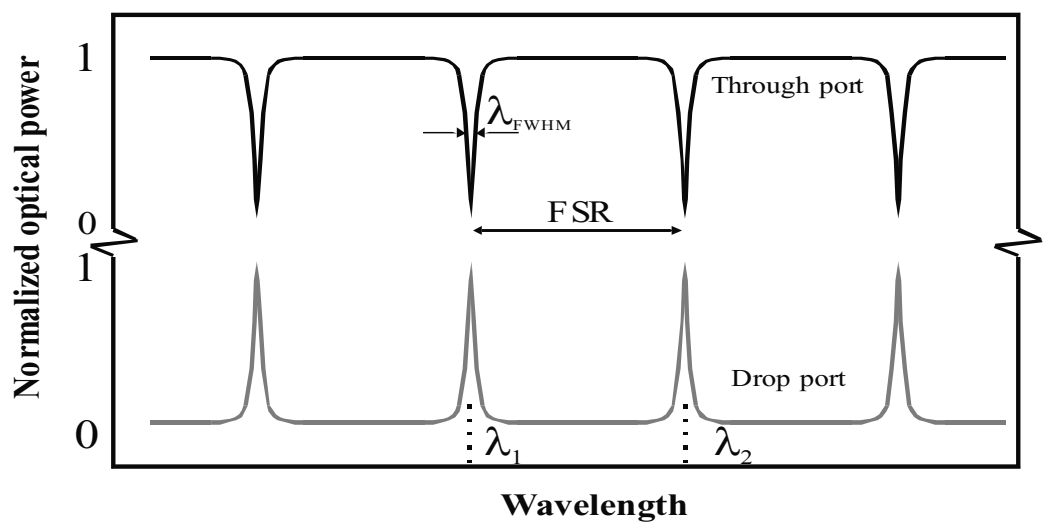

Figure 4.2: Schematic representation of the through and drop port response of a microring resonator.

The following important parameters which describe the behavior of a microring resonator are related as explained below [11].

The FSR of a microring resonator can be expressed as:

$\mathrm{FSR}=\lambda_{0}^{2} / 2 \pi \mathrm{Rn}_{\mathrm{g}}$

where, $\lambda_{0}$ is a resonance wavelength, $\mathrm{R}$ is the radius of the microring, and $\mathrm{n}_{\mathrm{g}}$ is the group index of the mode in the microring [12],

$\mathrm{n}_{\mathrm{g}}=\mathrm{n}_{\mathrm{e}}\left(\lambda_{0}\right)-\lambda_{0}\left|\mathrm{dn}_{\mathrm{e}} / \mathrm{d} \lambda\right|_{\lambda_{0}}$

in which $n_{e}$ is the effective index of the mode in the microring and $d n_{e} / d \lambda$ is the dispersion. The FSR is determined by the ring radius for a given waveguide geometry and materials choice.

The finesse of the microring is defined as

$\mathrm{F}=\mathrm{FSR} / \Delta \lambda_{\mathrm{FWHM}}$ 
which is the number of FWHM which can fit in one FSR. The number of round trips (m) which a photon makes before its energy is reduced to $1 / \mathrm{e}$ is given by [13]:

$\mathrm{m}=\mathrm{F} / 2$ л

For a microring resonator to be used as an electro-optic modulator, it is necessary to have a steep response, i.e. a small $\Delta \lambda_{\mathrm{FWHM}}$ and consequently a high finesse and large $\mathrm{m}$. That means that a low loss ring is required to have a steep ring response and low operating voltage. Another factor which influences the finesse is the coupling constant. The finesse increases as the coupling to the ring reduces. Therefore a high finesse can only be achieved in rings with low loss and low coupling constants. But the maximum in the dip size is achieved when the coupling to the ring just equals the losses in the ring (critical coupling). So as the ring losses increase, more power needs to be coupled to the ring to have the maximum possible dip size at some sacrifice of the finesse. Another parameter is the quality factor of the ring, which is an indication of the wavelength selectivity of the ring given by,

$\mathrm{Q}=\lambda / \Delta \lambda_{\mathrm{FWHM}}$

\section{1.2 Microring losses}

Different kinds of loss mechanisms are possible in a microring resonator. They can be broadly classified as,

1. Absorption losses

2. Scattering losses

3. Radiation losses

4. Modal mismatch losses

Absorption losses in the materials used by us arise mainly because of vibrational overtones of atomic bonds. In the communication wavelength range, this is dominated by $\mathrm{O}-\mathrm{H}$ and $\mathrm{C}-\mathrm{H}$ vibrational overtones [14]. Scattering losses can be sub divided into surface scattering and volume scattering losses [9]. Surface scattering arises from the fabrication imperfections and the volume scattering from defects in the material, for example, aggregates of molecules or dust particles. Surface scattering depends on the refractive index contrast between the core and the cladding. The same amount of the surface roughness can lead to high light scattering if the refractive index contrast increases, provided that the power density at the interface is the same. Radiation loss occurs when light propagates through a bend waveguide [15]. The amount of light radiated depends on the refractive index contrast between the waveguide and the surrounding. Modal mismatch losses occur at the coupling section because of unequal effective indices between the straight waveguide and the system of coupled waveguides. In addition transition losses between the straight and the bend sections of the waveguide also occur in the case of racetrack resonators. 


\subsubsection{Bandwidth limitations}

The microring resonator needs some time to build up the optical field. The time needed for one roundtrip $\tau_{\text {roundtrip }}$ is related to the ring radius $R$ by:

$\tau_{\text {roundtrip }}=2 \pi R / \mathrm{v}_{\mathrm{g}}=2 \pi R n_{\mathrm{g}} / \mathrm{c}$

where $\mathrm{v}_{\mathrm{g}}$ is the group velocity of light in the microring and $\mathrm{c}$ in vacuum. In order to build up power in a microring, multiple round trips are needed. The characteristic time needed for the microring to build up the maximum field at resonance [13] is expressed by:

$\tau_{\text {cav }}=\mathrm{m} . \tau_{\text {roundtrip }}=\mathrm{m} .2 \pi \mathrm{Rn}_{\mathrm{g}} / \mathrm{c}=\mathrm{FRn}_{\mathrm{g}} / \mathrm{c}$

therefore,

$\tau_{\text {cav }}=\lambda Q / 2$ лс

This imposes the limitation of the maximum possible modulation speed [16].

\section{2 Microring design}

The design is made for laterally coupled microring resonators. This geometry was chosen as its realization is a relatively simple process involving no critical alignment steps.

The design is based on inverted ridges made in the bottom cladding. In a second step these inverted ridges are filled with the core layer material. It is not possibly to fill selectively the inverted ridge without depositing a uniform slab layer outside the ridges. Therefore the design had also to deal with this residual slab to determine the maximum allowable slab height before the device performance is affected. The ring and the bus waveguides are made of PMMA-DR1 (PolyMethylMethAcrylate-DisperseRed-1) polymer which has a refractive index of 1.606 at $1550 \mathrm{~nm}$. The cladding layers were made of an UV curable epoxy VSC [17] with a refractive index of 1.501 at $1550 \mathrm{~nm}$. The cross section of the device is shown in Fig. 4.3.

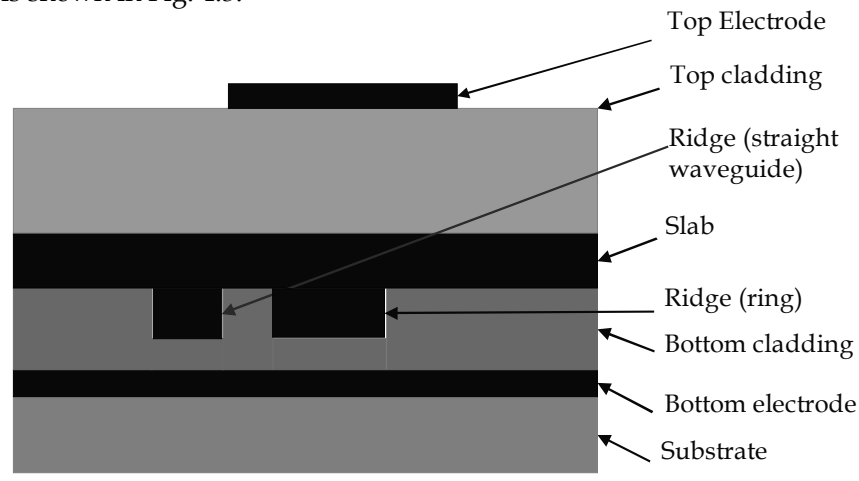

Fig 4.3: Cross-section of the coupling section of our laterally coupled ring resonators. 
In the design procedure the following parameters have to be determined for the ring and straight waveguide.

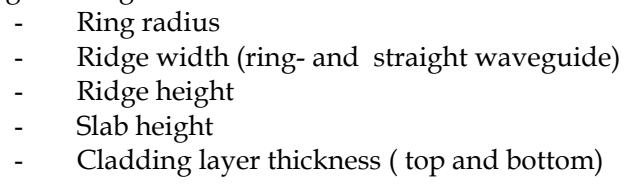

The dimensions of the straight waveguide for monomodal operation were obtained by simulations using TempSelene software [18]. The maximum ridge width of the straight waveguide which allows monomodal operation at $1550 \mathrm{~nm}$ was determined for different ridge heights. Fig. 4.4 shows the monomodal and multimodal operational regions for TE and TM polarizations. The height of the residual slab was taken to be zero in this case.

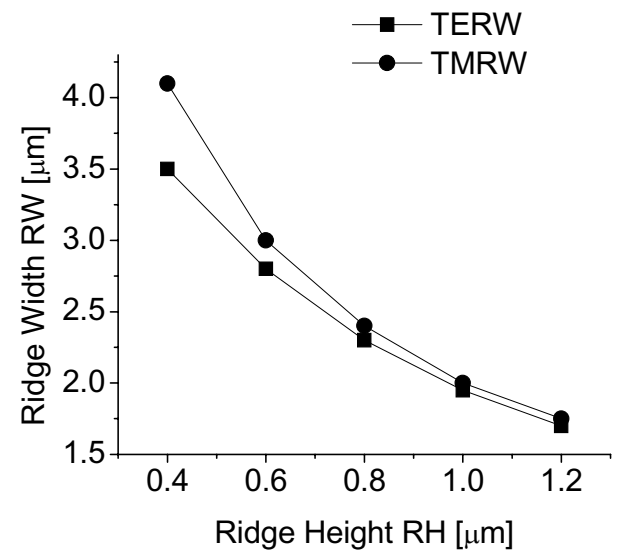

Figure 4.4: Maximum ridge width as a function of the ridge height for TE (TE RW) and TM (TM $R W)$ for monomodal operation.

Similar simulations were made by varying the slab height and the effective indices at the bimodal points (the limiting dimension at which a second mode starts to appear in the simulations) were determined. This is shown in Fig. 4.5. The effective indices of the straight waveguide modes are important for phase matching with the ring mode [19, 20]. The monomodal operation regime of the microring was also determined in a similar manner using the $2 \mathrm{D}$ bend mode solver of TempSelene. When the straight waveguide is bent in the form of a ring a part of the light in the waveguide mode is radiated out as the total internal reflection condition is affected. This effect increases as the radius of the bend decreases. The refractive index contrast between the core and the cladding sets the minimum bend radius before the radiation losses exceed a certain value. To determine the ring radius for which the losses due to bending of the waveguide are still acceptable, the radiation losses in the bends were simulated for different ring radii for the material system of our choice. 


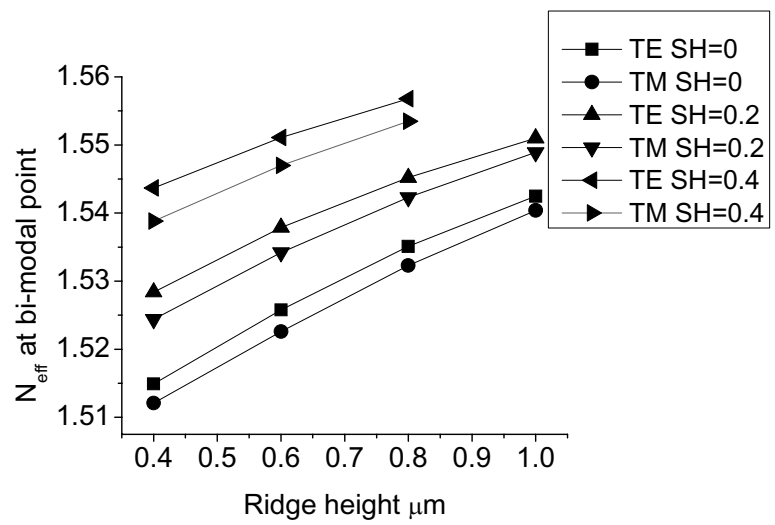

Figure 4.5: Effective index of the straight waveguide mode at the bi-modal point as a function of ridge height for different slab heights $\mathrm{SH}$.

Fig. 4.6 shows the radiation loss as a function of ring radius for different slab heights. An exponential decrease in the radiation loss with increase in ring radius can be seen. It also appears that the residual slab has a detrimental effect on the radiation losses. A ring radius is acceptable as long as its bend loss is relatively low compared with the losses caused by scattering and absorption. The maximum allowable bend loss therefore is about $1 \mathrm{~dB} / \mathrm{cm}$. Absorption and scattering losses in the ring are usually much higher than this value. As the radius of the ring becomes higher the round trip loss of the ring will increase because of absorption and scattering. Hence the radius of the ring should be chosen in such a way as to achieve a minimum in the round trip loss.

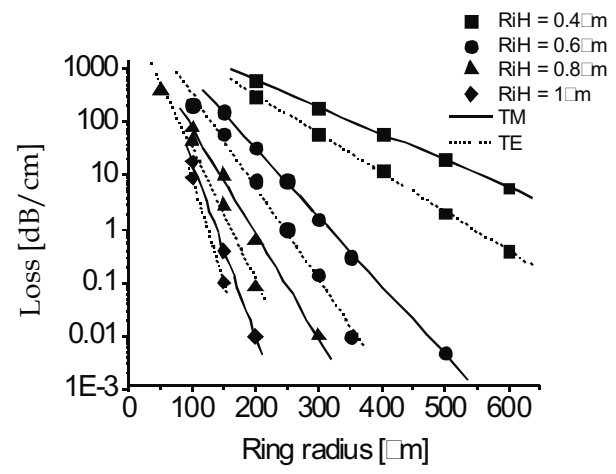

Figure 4.6 Radiation losses as a function of ring radius for different ridge heights of the ring waveguide [RiH]. 
The width of the ring should be such that the ring mode will be in the whispering gallery mode regime [21] and sense only the outer side wall of the ring. This will help in reducing the scattering losses which is a major source for the ring loss. Simulations were done to study the effect of the ring width on the $\mathrm{N}_{\text {eff }}$ of the mode. A ring radius of $200 \mu \mathrm{m}$ was chosen in this case. The results are shown in Fig. 4.7. For ring widths greater than $6 \mu \mathrm{m}$, it can be seen that the $\mathrm{N}_{\text {eff }}$ remains constant. Taking fabrication tolerances into account a width of $7 \mu \mathrm{m}$ was chosen for the ring.

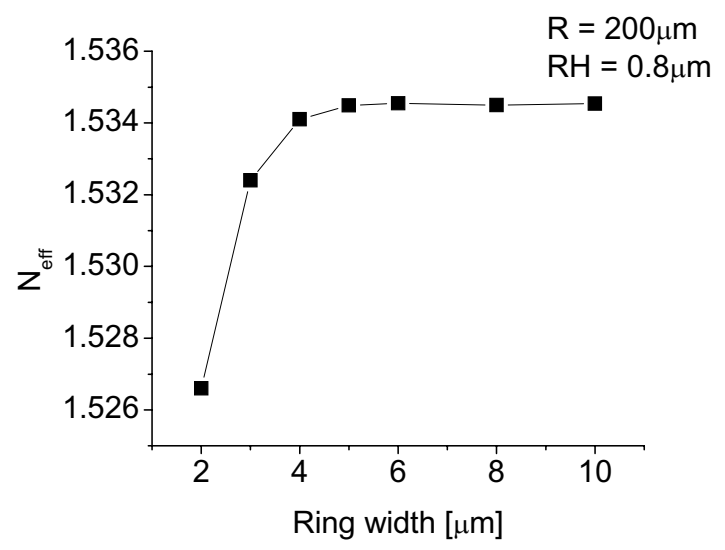

Figure 4.7: Effect of ring width on the mode effective index, the ridge height is $0.8 \mu \mathrm{m}$.

Table 4.1: Dimensions for the ring with a minimum radius and the straight waveguide satisfying monomodality of the straight waveguide and $1 \mathrm{~dB} / \mathrm{cm}$ radiation loss for the ring.

\begin{tabular}{|l|l|l|l|}
\hline \multicolumn{1}{|c|}{ Ridge height $[\mu \mathrm{m}]$} & Ridge width $[\mu \mathrm{m}]$ & Ring radius $[\mu \mathrm{m}]$ & Ring width $[\mu \mathrm{m}]$ \\
\hline 0.5 & 2.4 & 400 & 7 \\
\hline 0.6 & 2.2 & 300 & 7 \\
\hline 0.8 & 1.9 & 200 & 7 \\
\hline 1 & 1.8 & 150 & 7 \\
\hline 1.2 & 1.5 & 100 & 7 \\
\hline
\end{tabular}

For each set of the dimensions given in Table 4.1 phase matching can be obtained by fine tuning the radius of the ring and the waveguide width. In Fig. 4.8 the effective index of the ring mode and the straight waveguide mode are overlaid for comparison. As the ring and the straight waveguide are laterally coupled they have the same height, in our case fixed to $0.8 \mu \mathrm{m}$. In the case of the ring its radius is varied keeping the ring width constant at $7 \mu \mathrm{m}$. For the straight waveguide variation is provided in the ridge width. The minimum width of the straight waveguide was restricted to be $1 \mu \mathrm{m}$ as it is difficult to resolve structures of dimensions less than $1 \mu \mathrm{m}$ by standard contact photolithography. 


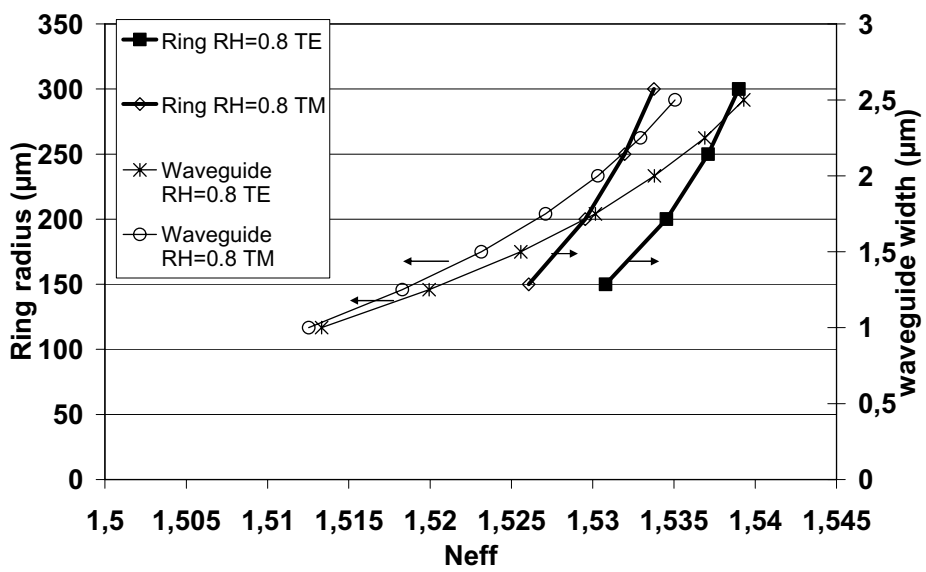

Figure 4.8: Ring radius and waveguide width of the straight waveguide for a given $N_{\text {eff }}$ for TE as well as TM. In all cases the ridge height is $0.8 \mu \mathrm{m}$.

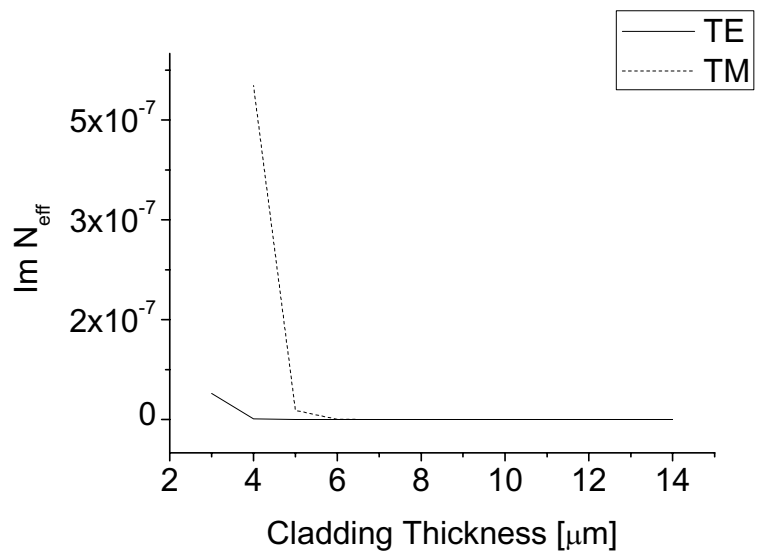

Figure 4.9: Imaginary index of the waveguide mode as a function of cladding layer thickness with an absorbing electrode on top.

The next step in the device design is to find out the optimum thickness of the cladding layers. The cladding layers have to be thick enough that the mode field will not sense the bottom and the top electrodes as otherwise it will lead to a high absorption loss. On the other hand reduced thickness of the cladding layers will favor low operation voltage of the device. To determine the optimum thickness simulations were done with an electrode 
placed on top of the waveguide. The electrode is assumed to have the same real part of the index as the cladding layer and an imaginary part of 10. By moving the electrode close to the waveguide a change in the imaginary part of the waveguide mode can be seen. The result of this simulation is shown in Fig. 4.9. At a certain value of the cladding layer thickness a sharp change in the imaginary part of the refractive index of the waveguide mode can be seen. This sets the minimum required thickness of the cladding layers. From Fig. 4.9 the thickness of the cladding layers was chosen to be $6 \mu \mathrm{m}$. 3D BPM simulations were made to have an estimate of the coupling constants between the ring and the straight waveguide for different ring radii. The results are presented in Fig. 4.10.

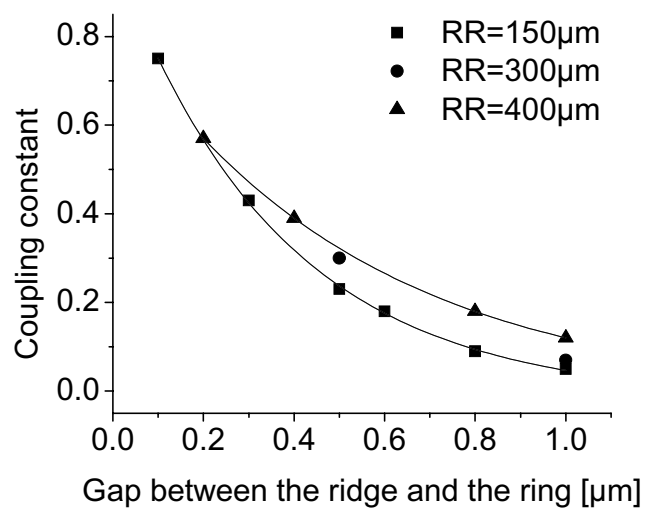

Figure 4.10 Simulated coupling constants as a function of the gap between the ring and the straight waveguide for several ring radii [RR].

\subsection{Fabrication methods}

Different fabrication methods were tried to realize inverted ridges in the polymer cladding, namely, photodefinition, embossing and reactive ion etching. Once realized, these inverted ridges are intended to be filled with PMMA-DR-1 acting as the waveguide core layer for the ring structure. The electro-optic PMMA-DR1 and most of the other linear polymers are attacked by the solvents used for the photoresist and also by the cladding layer solvents. In this way the realization of high resolution structures becomes very difficult. Therefore it is more convenient to define the structures in a crosslinkable cladding layer, as crosslinking makes the layer resistant to solvents.

\subsubsection{Photodefinition}

The following composition was used for photodefinition of the inverted ridges in VSC. A detailed description of the photodefinition process is given in Chapter 5 . 
VSC-6.7

UVR (3,4-epoxycyclohexane-carboxylate)-1.6 (UVR 6110 of Union carbide)

CP-12.5 (Cyclopentanone)

$4 \% \mathrm{UVI}$

This composition gives a photodefinable materials system similar to commercially available SU8 but with a lower refractive index. SU8, because of its high refractive index, cannot be used as a cladding layer material. VSC is an epoxy resin which forms solid films before crosslinking. This enables the usage of contact mask during exposure. UVR is also an epoxy resin with a higher number of epoxy groups than VSC which will yield a high crosslinking density. A high crosslinking density is better to define high resolution structures. But it does not form a solid film suitable for exposure using a contact mask. Hence a formulation consisting of a mixture of VSC and UVR is made to combine the advantages of both VSC (solid film before crosslinking) and UVR (high crosslinking density). Cyclopentanone is used as the solvent and $4 \%$ UVI as a photoinitiator.

The ring opening process of the epoxy groups is catalyzed by a strong acid generated by the decomposition of a photoinitiator. This photonitiator should be active for the operational wavelength of the exposure apparatus. Photoinitiators for the most common Iline mask aligners are readily available. Onium salts based on triarylsulfonium hexafluoroantimonate are widely used, including in SU-8, because of their effectiveness [22]. However, in our formulation, we used triarylsulfonium hexafluorophosphate (UVI 6992 of Union Carbide), because this yields less yellowing on post-baking. An amount of 4 $\mathrm{wt} \%$ is used, which is twice as much as is typically used in SU-8.

In the post-exposure bake, the photoacid-catalyzed epoxy crosslinking takes place, resulting in insolubility of the latent photoacid image in the polymer which will not be washed away in the subsequent development step. The solution was stirred overnight in a stirrer and filtered using a $0.2 \mu \mathrm{m}$ filter. A layer of $5 \mu \mathrm{m}$ thickness was spin coated on a silicon wafer, baked at $95^{\circ} \mathrm{C}$ to remove the solvents, exposed to UV and cured at $95^{\circ} \mathrm{C}$ for 10 mins. A layer of $1 \mu \mathrm{m}$ thickness was spin coated on top of this, baked at $95^{\circ} \mathrm{C}$ to remove the solvents and exposed to UV using a bright field mask. The layer was then cured at $95^{\circ} \mathrm{C}$ for 10 mins. The film was then developed. The unexposed region is removed during developing. The exposed regions surrounding the waveguides are crosslinked and remain after developing. Several trial experiments were made to optimize the exposure time and curing temperature.

The best results obtained are shown in Fig. 4.11. It was not possible to clearly open inverted ridge waveguides less than $3 \mu \mathrm{m}$ wide. Looking at Table 4.1 for a waveguide width of $2.4 \mu \mathrm{m}$ and waveguide height of $0.5 \mu \mathrm{m}$ it requires a ring radius of $400 \mu \mathrm{m}$ for phase matching and to keep the radiation loss of the ring below $1 \mathrm{~dB} / \mathrm{cm}$. 

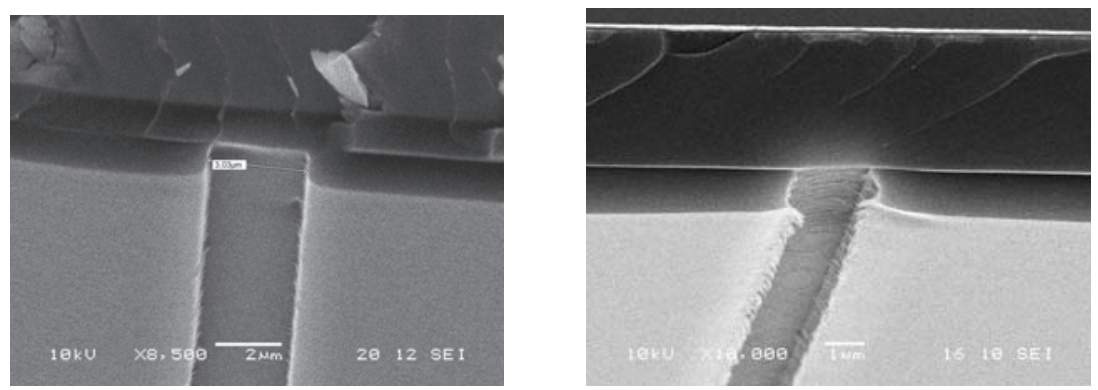

Figure 4.11: Inverted ridges with a width of approximately $3 \mu \mathrm{m}$ made by photodefinition in VSC.

\subsubsection{Embossing}

In the case of photodefined inverted ridges in VSC, the waveguide width was restricted to a minimum of $3 \mu \mathrm{m}$. In order to reduce this width also an embossing technique was tried. The composition of the bottom cladding used for photodefinition was modified for the purpose of embossing. Only UVR was used without VSC, as UVR forms soft films in the uncrosslinked state which can easily be embossed. A thermal initiator was used instead of the photoinitiator. The schematic of the embossing process is shown in Fig. 4.12. The master for embossing was made in $\mathrm{SiO}_{2}$ on a $\mathrm{Si}$ substrate by photolithography followed by RIE. It was coated with a thin layer of anti-sticking agent. The UVR formulation was spin coated on a Si substrate and baked to remove the solvents at $95^{\circ} \mathrm{C}$. The master was pressed against the film with a load of $2 \mathrm{Kg}$ applied on the 4 inch wafer. The wafer together with the master was heated to $95^{\circ} \mathrm{C}$ to thermally crosslink the UVR layer. The master was then removed leaving the inverted ridge structure on the UVR layer. It was possible to make waveguides of width of $1 \mu \mathrm{m}$ by this method. However it was very difficult to remove the master after embossing. Even the applied antisticking layer did not seem to have much effect in avoiding this sticking problem. The SEM picture of an embossed inverted ridge is shown in Fig. 4.13. The sticking problem was so adverse that the results were not reproducible. 


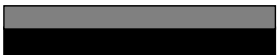

Master Oxide wafer

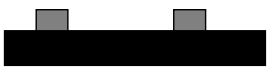

RIE

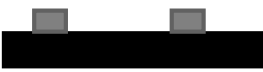

Antisticking layer

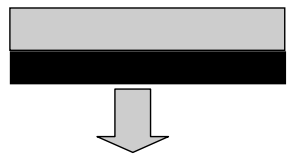

VSC with thermal

initiator

Hot plate

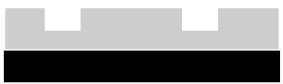

Figure 4.12: Schematic of the embossing process.

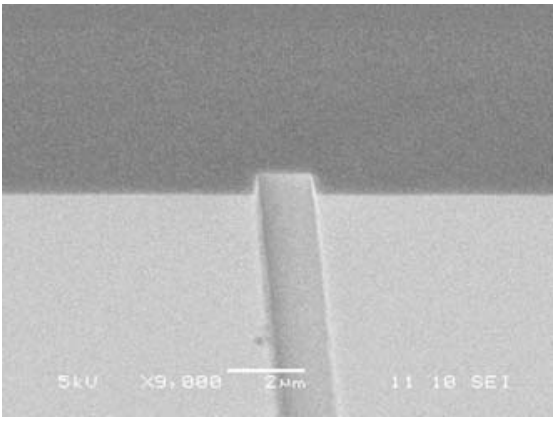

Figure 4.13: SEM picture of an embossed inverted ridge in UVR.

\subsubsection{Reactive ion etching in VSC}

Inverted ridges in the bottom cladding could also be obtained by RIE with $\mathrm{O}_{2}$ as reactive gas. For the bottom cladding a formulation was used similar to the one for inverted ridges made by photodefinition but now only with VSC. UVR was not used in this case as a high crosslinking density was not required here. A $6 \mu \mathrm{m}$ layer of the VSC was spin coated on 
the Si substrate baked, exposed to UV and cured at $95^{\circ} \mathrm{C}$. Photolithography was carried out using a dark field mask with the microring design (for positive resist) in the contact mode. The mask consists of rings with varying radius from $100 \mu \mathrm{m}$ until $400 \mu \mathrm{m}$. The gap between the straight waveguide and the ring was given a variation from $1 \mu \mathrm{m}$ to $3 \mu \mathrm{m}$. Waveguide widths down to $1 \mu \mathrm{m}$ were easily achievable by RIE. The minimum resolvable gap size between the straight waveguide and the ring was $2 \mu \mathrm{m}$. Gaps of less than $2 \mu \mathrm{m}$ could not be opened by RIE. The inverted ridges were filled with PMMA-DR1, which performs as waveguide core. First single step spinning was tried with a thick solution resulting, however, in insufficient gap filling properties. Fig. 4.14 shows an improperly filled inverted ridge when a thick PMMA-DR1 solution was spin coated in a single step. Thereafter the spinning of the PMMA-DR1 layer was done in two steps using a thin solution. After spinning for the second time a thin slab layer was found also outside the inverted ridge region. This layer was removed by an additional RIE step. Fig. 4.15 shows an optical microscope picture of the resulting microring and the bus waveguides. Fig. 4.16 shows the SEM picture of the cross section of the device with a $6 \mu \mathrm{m}$ layer of VSC as top cladding. The wafer was diced so that the devices could be measured. Straight waveguides functioned well but no fringes of the microrings could be observed probably because of insufficient coupling between ring and straight waveguide. For better coupling the gap between the ring and straight waveguide should be well below the actual $2 \mu \mathrm{m}$.

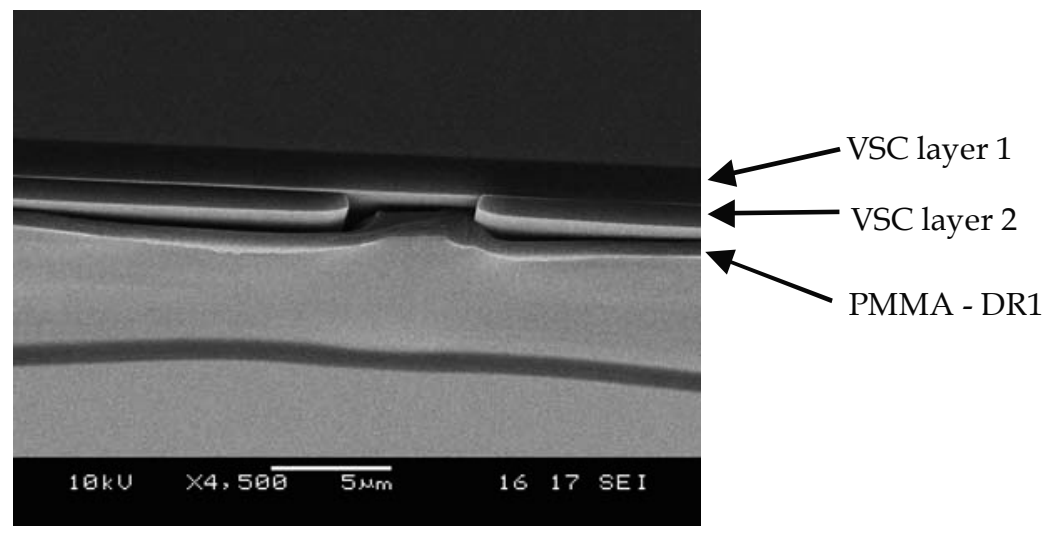

Figure 4.14 SEM picture of inverted ridge in VSC filled with PMMA-DR1 showing improper filling of PMMA-DR1. 

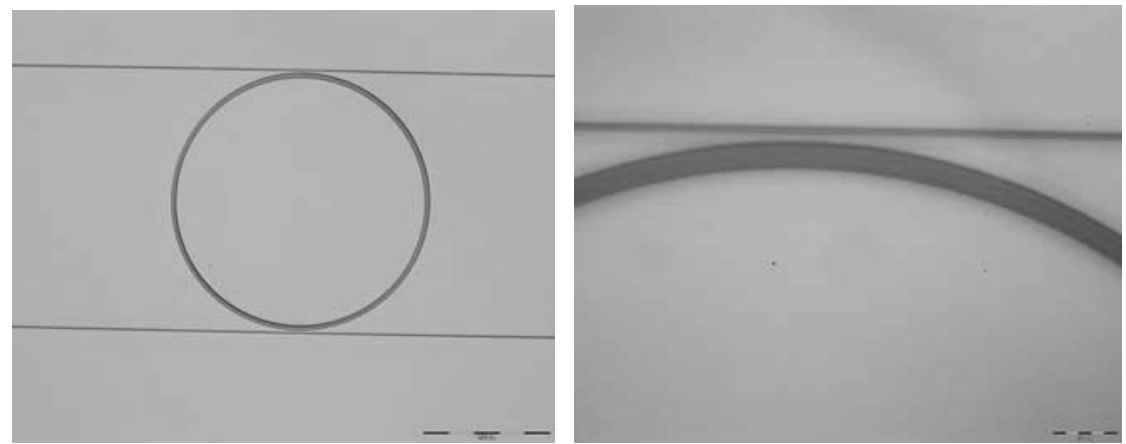

Figure 4.15 Microscope picture of a PMMA-DR1 microring resonator with a radius of $200 \mu \mathrm{m}$ fabricated by filling inverted ridges made by RIE in the VSC bottom cladding.

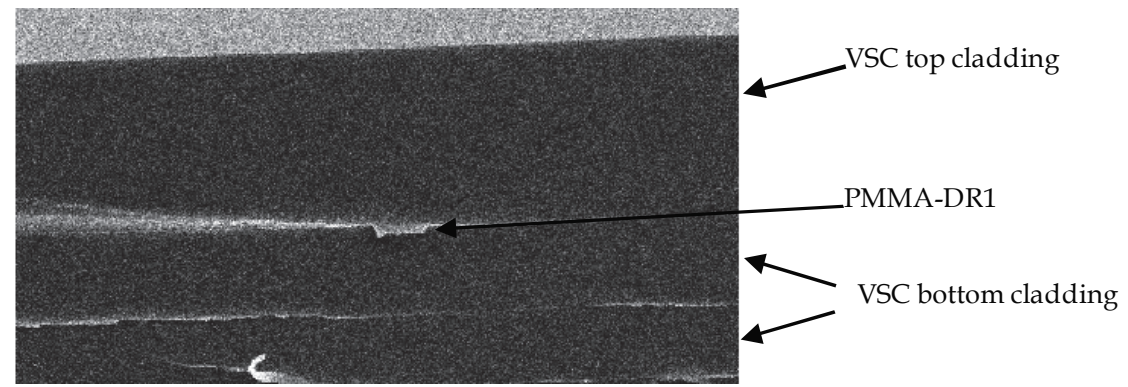

Figure 4.16: SEM picture of the cross section of an inverted ridge waveguide made by RIE with a PMMA-DR1 core and VSC top cladding.

\subsubsection{Inverted ridges in $\mathrm{SiO}_{2}$ - hybrid approach}

In order to improve the resolution in the gap region inverted ridges were defined in silicon oxide by RIE and then filled with polymer by spinning. The residual slab layer outside the inverted ridges was reduced to zero by RIE. This approach for fabricating microrings was chosen because of the following reasons.

1. Photodefinition of inverted ridges in a VSC bottom cladding did not give good results. Inverted ridges of less than $3 \mu \mathrm{m}$ could not be defined even after extensive optimization of the photodefinition process.

2. Embossing experiments in VSC bottom cladding gave serious sticking problems which could not be solved even after applying an antisticking layer to the master. 
3. Inverted ridges were defined in VSC by RIE. A gap of $2 \mu \mathrm{m}$ can be opened between the ring and the straight waveguide. But no ring response was observed because of poor coupling between the ring and the straight waveguide.

Photolithography and RIE in $\mathrm{SiO}_{2}$ is a well established process. Using this approach inverted ridges were defined in thermally grown $\mathrm{SiO}_{2}$ on $\mathrm{Si}$. A gap of $1 \mu \mathrm{m}$ could be opened by this method between the straight waveguide and the ring. These inverted ridges were filled with PMMA-DR1 in two layers and the excess slab height was reduced to zero by RIE. A $6 \mu \mathrm{m}$ layer of VSC was spin coated as the top cladding, exposed to UV and cured. The complete process flow and the exact processing parameters are given in the appendix.

\subsubsection{Measurements of the microring made by the hybrid approach}

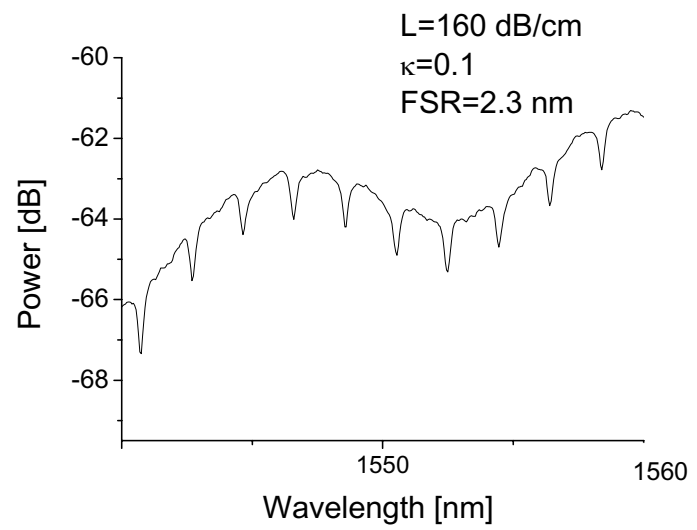

Figure 4.17: Through-port response of a PMMA-DR1 microring of $100 \mu \mathrm{m}$ radius. Also the extracted values of ring loss (L), FSR and coupling constant $(\kappa)$ are shown.

The processed wafer consisted of rings of radius varying from $150 \mu \mathrm{m}, 200 \mu \mathrm{m}, 300 \mu \mathrm{m}$ and $400 \mu \mathrm{m}$. The wafer was diced and the ring responses were measured. The microring devices were tested by coupling in TE polarized light from an Erbium doped fiber amplifier (emission from $1530 \mathrm{~nm}$ to $1560 \mathrm{~nm}$ and $10 \mathrm{~mW}$ output power). The out-coupled light was analyzed with a spectrum analyzer. The ring response of rings with different ring radii and $1 \mu \mathrm{m}$ gap is shown in Figures 4.17 to 4.20 . 


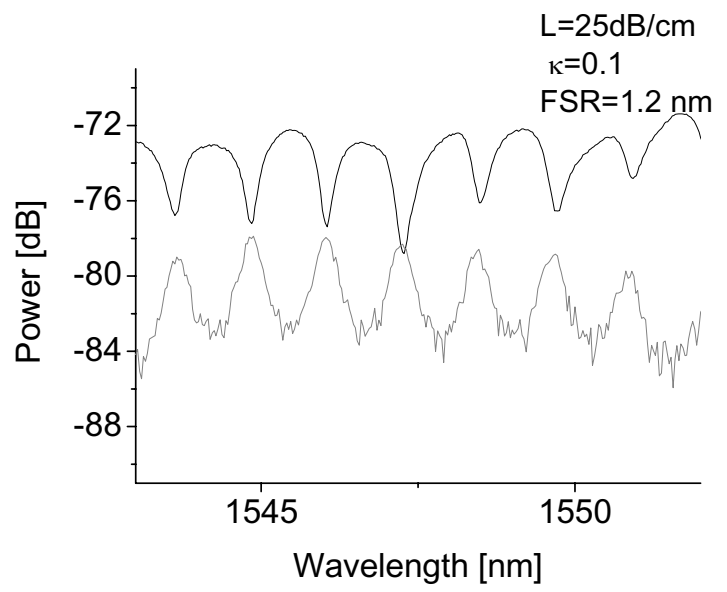

Figure 4.18: Through-port and drop-port response of a PMMA-DR1 microring of $200 \mu \mathrm{m}$ radius. The extracted values of ring loss $(L), F S R$ and coupling constant $(\kappa)$ are shown.

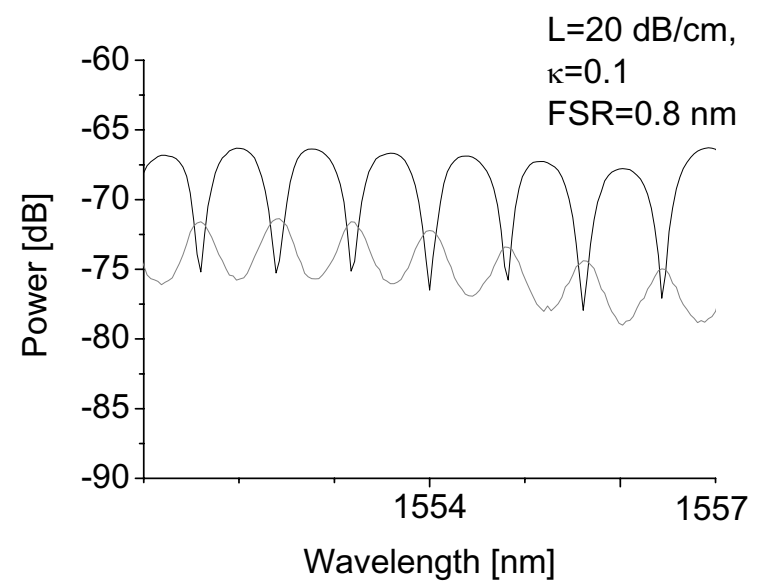

Figure 4.19: Through-port and drop-port response of a PMMA-DR1 microring of $300 \mu \mathrm{m}$ radius. The extracted values of ring loss $(L), F S R$ and coupling constant $(\kappa)$ are shown. 
The ring loss and coupling constant were extracted by making a fit to the through port response using Rfit software [23]. From these values the roundtrip losses of the rings were calculated and plotted as a function of the ring radius in Fig. 4.21. It can be seen that the minimum in the roundtrip loss of the ring occurs at a radius of about $200 \mu \mathrm{m}$. For ring radii lower than this the round trip loss sharply increases mainly because of increase in the radiation loss. At radii larger than $220 \mu \mathrm{m}$ the increase in the roundtrip loss is small and linear with the roundtrip length. In this regime the absorption and scattering loss is the dominant factor for the increase in the roundtrip loss. At ring radii lower than about $220 \mu \mathrm{m}$ radiation losses dominates the overall roundtrip loss of the ring. The measured and simulated values of the coupling constants are shown in Fig. 4.22. The measured values are slightly higher than that of the simulated values at larger ring radius. The reason for this is that at larger ring radius some amount of coupling occurs also outside the simulation window.

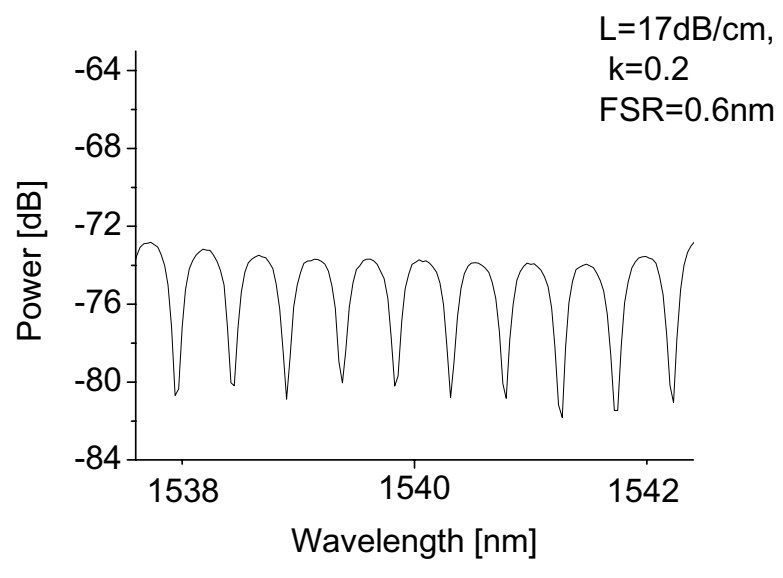

Figure 4.20: Through-port response of a PMMA-DR1 microring of $400 \mu \mathrm{m}$ radius. The extracted values of ring loss $(L)$ and coupling constant $(\kappa)$ are shown. 


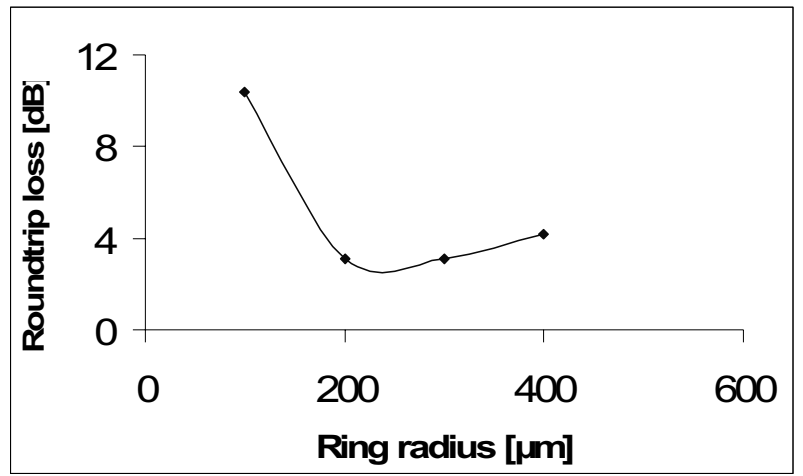

Figure 4.21: Roundtrip loss of the ring for different ring radii.

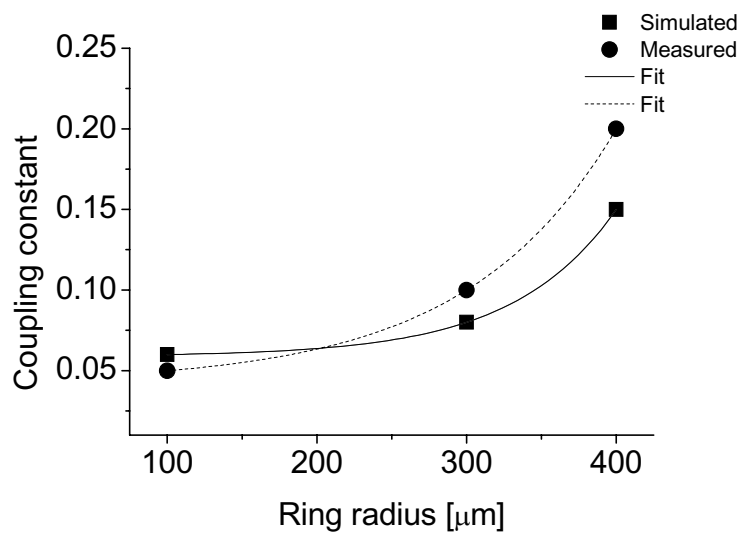

Figure 4.22: Simulated and extracted values (from the measurements) of the coupling constants obtained from PMMA-DR1 rings of different ring radius, for a constant gap of $1 \mu \mathrm{m}$.

\subsection{Racetrack resonator}

\subsubsection{Racetrack design}

It can be seen from Fig. 4.22 that for the ring radius of $200 \mu \mathrm{m}$, for which the roundtrip loss of the ring is near to minimum, the coupling constant is only about 0.06 for the gap of $1 \mu \mathrm{m}$. The through port dip in this case was about $4 \mathrm{~dB}$. Further increase in the through port dip can only be obtained by increasing the coupling to the ring. As a gap size of less than $1 \mu \mathrm{m}$ was not possible to fabricate using photolithography and RIE, an alternative 
design is made. It consists of a racetrack-shaped structure with a straight section of the ring in order to increase the coupling between the ring and the straight waveguide [8]. A schematic of this design is shown in Fig. 4.23.

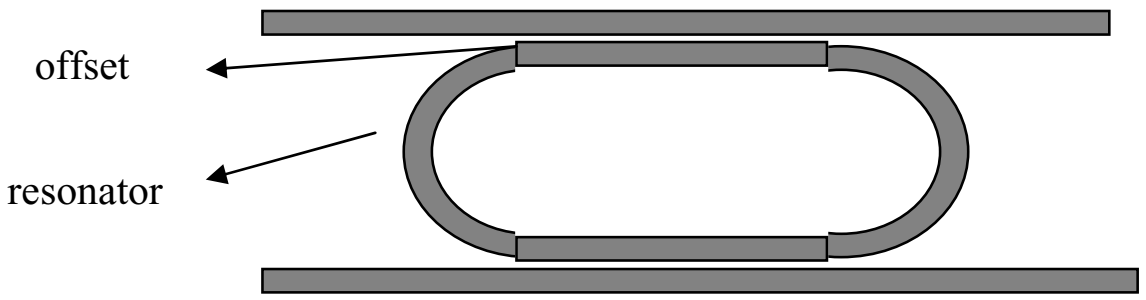

Figure 4.23: Schematic of the racetrack design.

Materials:

Core:

Cladding:VSC

$$
\begin{aligned}
& \text { PMMA-DR1 n=1.606@1550 nm } \\
& \mathrm{n}=1.5 @ 1550 \mathrm{~nm}(6 \mu \mathrm{m})
\end{aligned}
$$

\section{Straight waveguide dimensions:}

Waveguide width: $2 \mu \mathrm{m}$

Waveguide height: $1 \mu \mathrm{m}$

\section{Ring waveguide dimension:}

$\begin{array}{ll}\text { Bend width: } & 3 \mu \mathrm{m} \\ \text { Bend height } & 1 \mu \mathrm{m} \\ \text { Bend radius: } & 200 \mu \mathrm{m}\end{array}$

A ring radius of $200 \mu \mathrm{m}$ was chosen for the design as this value is close to the minimum in the roundtrip loss of the ring, see Fig. 4.21. A bend width of $3 \mu \mathrm{m}$ was designed in order to better phase match the ring mode and the straight waveguide mode, which in the previous design was $7 \mu \mathrm{m}$ (whispering gallery regime).

\section{Coupling section:}

The coupling length for the chosen geometry was simulated to be $200 \mu \mathrm{m}$. Hence the length of the straight section of the ring was varied from $20 \mu \mathrm{m}$ to $240 \mu \mathrm{m}$, in order to have a range of coupling constants. The waveguide modes in the bend and the straight sections of the ring do not match as the bend waveguide mode is shifted to the outside of the ring. An offset of $0.1 \mu \mathrm{m}$ was provided between the bend and the straight section of the ring in order to minimize this mismatch. Overlap mismatch (transition) loss per transition was simulated to be $0.10 \mathrm{~dB}$. Therefore the transition loss contribution to total roundtrip loss would be $0.4 \mathrm{~dB}$ (equivalent to $4 \mathrm{~dB} / \mathrm{cm}$ ). 


\subsubsection{Racetrack fabrication by the hybrid approach}

The hybrid approach based on inverted ridges in $\mathrm{SiO}_{2}$ bottom cladding was adopted for the realization of the racetrack design. The same fabrication process as explained in section 4.1 has been followed. A microscope picture of the fabricated device before spinning of the top cladding is shown in Fig. 4.24. A gap of $1 \mu \mathrm{m}$ was clearly opened.

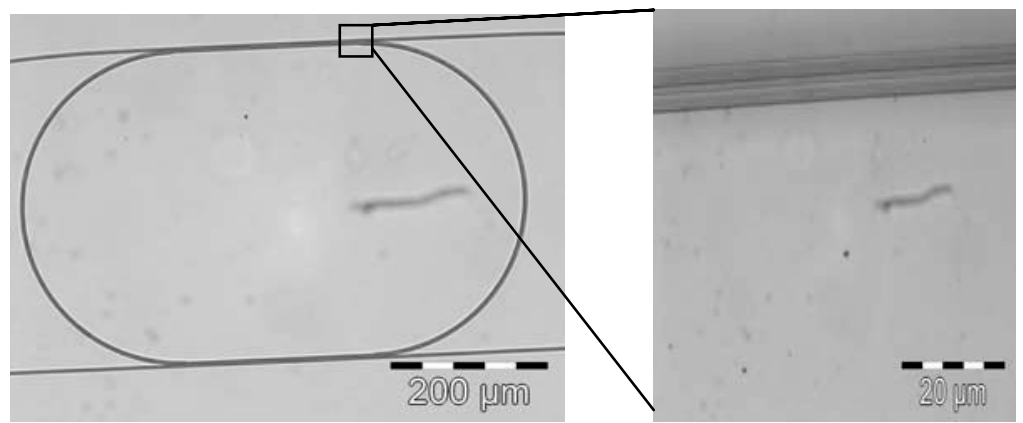

Figure 4.24: PMMA-DR1 racetracks fabricated by defining inverted ridges in silicon oxide by RIE; left: total view, right: detail of coupling section.

The microring devices were tested by coupling in TM polarized light from an Erbium doped fiber amplifier (emission from $1530 \mathrm{~nm}$ to $1560 \mathrm{~nm}$ and $10 \mathrm{~mW}$ output power). The out-coupled light was analyzed with a spectrum analyzer. Fig. 4.25 shows the through port response of the PMMA-DR1 microrings with a gap of $1 \mu \mathrm{m}$ for various racetrack lengths from $20 \mu \mathrm{m}$ to $160 \mu \mathrm{m}$ and Fig. 4.26 for the racetrack length from $180 \mu \mathrm{m}$ to 240 $\mu \mathrm{m}$. It can be seen from Fig. 4.25 that the extinction ratio increases with the racetrack length up to $160 \mu \mathrm{m}$. The highest extinction ratio of $12 \mathrm{~dB}$ was seen at a racetrack length of $160 \mathrm{~nm}$. With further increase in racetrack length coupling exceeds critical coupling and the extinction ratio decreases. The quality factor of the resonance was measured for the device with $160 \mathrm{~nm}$ racetrack to be 8000 and the finesse was 6 . The free spectral range varies from $1.1 \mathrm{~nm}$ to $0.9 \mathrm{~nm}$ as the racetrack length varies from $20 \mathrm{~nm}$ to $240 \mathrm{~nm}$. 


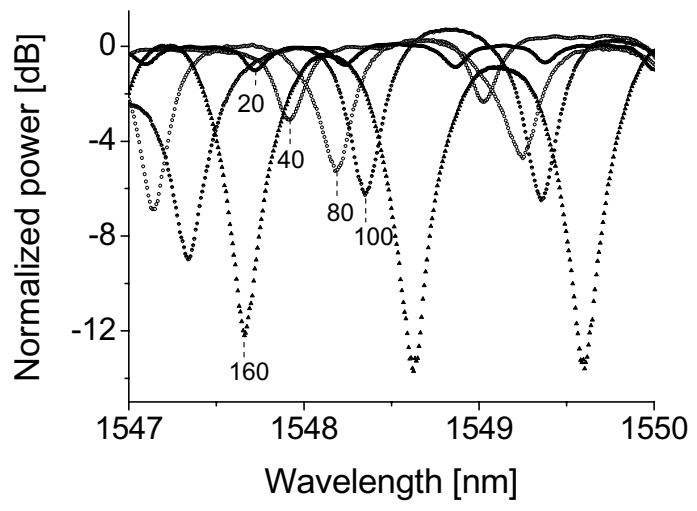

Figure 4.25: Through-port response of PMMA-DR1 racetracks for different lengths of the coupling section ranging from $20 \mu \mathrm{m}$ to $160 \mu \mathrm{m}$.

The coupling constants and the ring losses were extracted from the through port response by making a fit using Rfit software. The ring loss was found to be $30 \mathrm{~dB} / \mathrm{cm}$. The excess 5 $\mathrm{dB} / \mathrm{cm}$ loss compared with the ring design in Fig. 4.18 arises probably from the modal mismatch loss at the transition between the bend and the straight section of the ring. This is quite consistent with the simulated modal mismatch loss of $4 \mathrm{~dB} / \mathrm{cm}$.

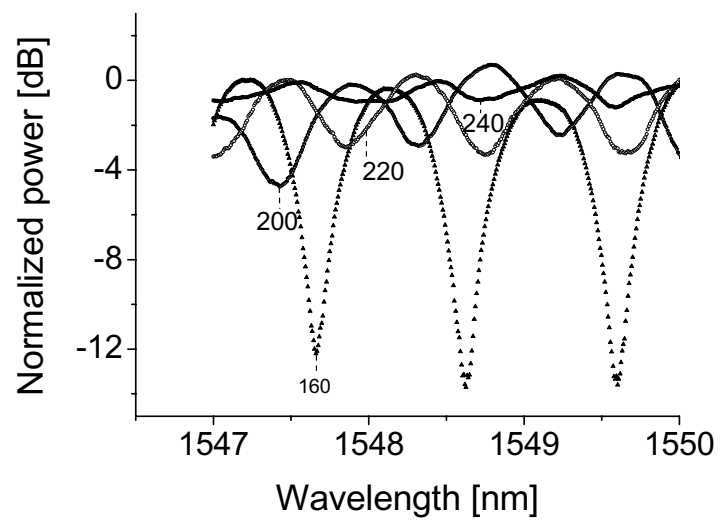

Figure 4.26: Through-port response of PMMA-DR1 racetracks for different lengths of the coupling section ranging from $160 \mu \mathrm{m}$ to $240 \mu \mathrm{m}$. 


\subsubsection{Poling of racetrack devices}

In a different run devices were made using the hybrid approach and the racetrack design for the purpose of poling the devices. A highly conductive silicon wafer was used as the substrate and also as the bottom electrode for poling. Aluminum contacts were made to the substrate for the contacting. The device was fabricated according to the scheme in appendix A. A $250 \mathrm{~nm}$ layer of gold was sputtered on the top cladding serving as the top electrode for poling. Poling was carried out by slowly heating the sample to $110^{\circ} \mathrm{C}$ which is close to the glass-transition temperature of PMMA-DR1. A DC voltage of about $1200 \mathrm{~V}$ was applied to the stack. Defects in the layer stack prevented further increase of the applied voltage. An electric field of $10 \mathrm{~V} / \mu \mathrm{m}$ was expected to be across the core layer. In order to have maximum poling efficiency an electric field of at least $100 \mathrm{~V} / \mu \mathrm{m}$ is required across the core layer. As the resistivity of $\mathrm{SiO}_{2}$ is very high compared with polymers, in plane poling using co-planar electrode configuration can result in high poling efficiencies. Fig. 4.27 and Fig 4.28 show the through port spectrum of a racetrack device before and after poling respectively. During poling the core layer becomes birefringent [24]. The refractive index in one of the polarization directions increases and in the other decreases. This makes the through port spectrum for the TE and TM to shift in opposite directions. This can be seen from Fig. 4.27 and Fig. 4.28. By making fits to the through-port spectra before and after poling, the poling induced losses were estimated to be about $2 \mathrm{~dB} / \mathrm{cm}$.

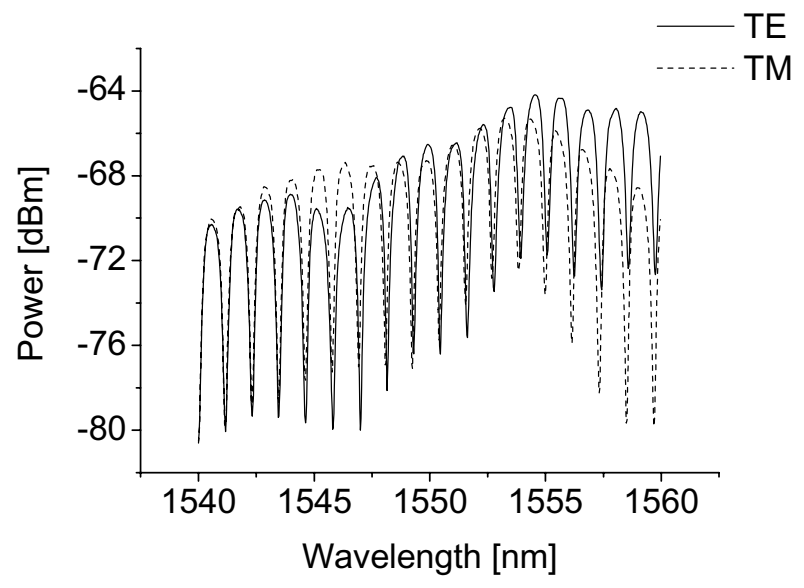

Figure 4.27: Through port response of PMMA-DR1 racetrack showing no birefringence between TE and TM modes before poling. 


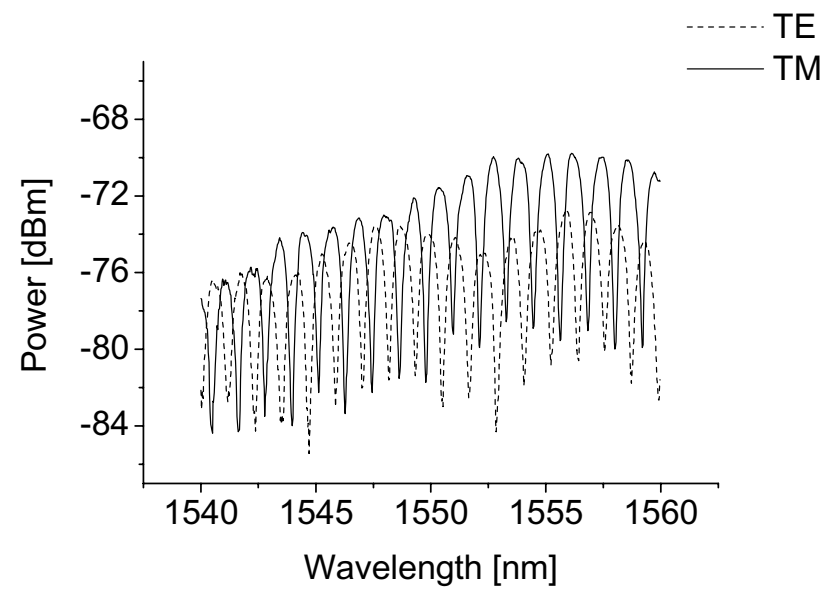

Figure 4.28: Through port response of PMMA-DR1 racetrack showing a shift between TE and TM caused by poling induced birefringence.

\subsection{Conclusions}

A design was made for laterally coupled microrings with PMMA-DR1 as the core material and VSC as the cladding layers. The minimum possible bending radius before the radiation losses increases above $1 \mathrm{~dB} / \mathrm{cm}$ was found to be $200 \mu \mathrm{m}$. Simulations showed that a sub micron gap between the ring and the straight waveguide is required to achieve the required coupling. With a racetrack design it is possible to increase the coupling between the ring and the bus waveguide at a gap of about $1 \mu \mathrm{m}$. Photodefinition of inverted ridges in VSC showed that it was not possible to make inverted ridges less than 3 $\mu \mathrm{m}$ wide and it was not possible to get the $\mu \mathrm{m}$-wide gap open. By embossing it was possible to open the $1 \mu \mathrm{m}$ gap, but removal of the master posed serious problems as it distorted the embossed structures. By using RIE inverted ridges of the ring design were able to be made with gap size not less than $2 \mu \mathrm{m}$ and hence no ring response could be measured because of insufficient coupling. The $1 \mu \mathrm{m}$ gap was made possible with the hybrid approach by defining inverted ridges in $\mathrm{SiO}_{2}$ by RIE and filling them with PMMADR1 by spin coating. The racetrack made by this approach showed a through port dip of $12 \mathrm{~dB}$ and a finesse of 6 . The ring losses were about $25 \mathrm{~dB} / \mathrm{cm}$.

\subsection{References}


[2] M. Pelton, C. Santori, J. Vuckovic, B. Zhang, G. S. Solomon, J. Plant, Y. Yamatoto, Phys. Rev. Lett., 89, p233602, 2002.

[3] Q. Xu, B. Schmidt, S. Pradhan, M. Lipson, Nature, 435, p325, 2005.

[4] P. Dong, S. F. Preble, M. Lipson, Opt. Express, 15, p9600, 2007.

[5] S. Xiao, M. H. Khan, H. Shen, M. Qi, J. of Lightwave Technol., 26(2), 2008.

[6] B. Miao, P. Yao, J. Murakowski, D. W. Prather, J. Microlith., Microfab., Microsyst., 4(2), p023013, 2005.

[7] C. Y. Chao, L. J. Guo, J. Vac. Sci. Technol., B 20(6), p2862, 2002.

[8] E. J. Klein, 'Densely Integrated Microring-Resonator Based Components for Fiber-ToThe-Home Applications', PhD Thesis, Integrated Optical Micro Systems group, University of Twente, the Netherlands, 2007.

[9] R. G. Hunspreger, Integrated Optics: Theory and Technol., Springer Series in Optical Sciences. Springer -Verlag, second edition, 1984.

[10] T. Tamir, Integrated Optics, vol. 7 of Topics in Applied Physics, Springer, Berlin, 1979.

[11] D. J. W. Klunder, Photon physics in intergrated optics microresonators, PhD Thesis, Integrated Optical Micro Systems group, University of Twente, Enschede, the Netherlands, 2002.

[12] C. K. Madsen, J. H. Zhao, Optical filter design and analysis, John Wiley \& sons inc., New York, 1999, ISBN: 047113733.

[13] J. T. Verdeyen, Laser electronics, third edition, Prentice Hall, New Jersy, ISBN: 013706666X, 1981.

[14] C. Pitois, A. Hult, D. Wiesmann, J. Opt. Soc. Am. B, 18(7), p908-912, 2001.

[15] E. C. M. Pennings, Bends in Optical Ridge Waveguides, PhD thesis, Delft University of Technology, 1990.

[16] A. Driessen, D.H. Geuzebroek E.J. Klein R. Dekker, R. Stoffer, C. Bornholdt, Optics Communications, 270, p217, 2007.

[17] From Surface Specialties Cytec Industries formerly UCB.

[18] Commercial product of C2V, colosseum 20, 7521 PT Enschede, The Netherlands. 
[19] D. J. W. Klunder, E. Krioukov, F. S. Tan, T. Van Der Veen, H. F. Bulthuis, G. Sengo, C. Otto, H. J. W. M. Hoekstra, A. Driessen, Appl. Phys. B, 73, p603, 2001.

[20] D. H. Geuzebroek, and Driessen, A., Ring-resonator-based wavelength filters In: Wavelength filters in fibre optics, Springer series in optical sciences Springer Verlag, Berlin/Heidelberg, 123, p341, 2006.

[21] F. C. Blom, Linear and non-linear optical properties of cylindrical micro-resonators: from material to device realization, PhD Thesis, Integrated Optical Micro Systems group, University of Twente, the Netherlands, 1998.

[22] J. V. Crivello, J. Polym. Sci. Part A: Polym. Chem., 37, p241, 1999.

[23] Non-commercial Microring fit software by E. J. Klein, Integrated Optical Microsystems group, University of Twente, The Netherlands.

[24] Horowitz, M. B. Pereira, P. Alcantara, H. P. Greineisen, V. Stefani, J. of Noncrystalline solids, 284, p183, 2001. 


\section{MR-based modulator made by direct photodefinition of an electro-optic polymer}

A laterally coupled microring resonator was fabricated by direct photodefinition of negative photoresist su8, incorporated with tricyanovinylidenediphenylaminobenzene chromophores, by exploiting the low uv absorption window of this chromophore. The ring resonator was first photodefined by slightly crosslinking. Thereafter poling (to align the chromophores) and further crosslinking (to increase the glasstransition temperature-Tg) was carried out simultaneously. The material showed excellent photostability and electro-optic modulation with an $r_{33}$ coefficient of $11 \mathrm{pm} / \mathrm{V}$ was demonstrated at $10 \mathrm{MHz}$.

Published as: M. Balakrishnan et al, Applied physics letters, 92 (15) Pp 153310-1 to 153310-3, 2008. 


\subsection{Introduction}

Several techniques have been reported for the fabrication of polymeric channel waveguides such as reactive ion etching [1], ion implantation [2], UV laser beam scanning [3], electron-beam lithography [4], and embossing techniques [5]. However these processes involve many processing steps, are time consuming, have low yield, are not yet far developed or are cost intensive. RIE for long has been used as a standard technique for structuring polymers in a cost effective manner, but does not allow definition of micronsized structures, see Section 4.3.3. Fabrication of waveguides by photobleaching [6, 7] and photo-crosslinking [8] has also been demonstrated. The maximum achievable refractive index contrast in these cases is not enough to make MRs with acceptable large FSR. Direct photodefinition is advantageous than these techniques. But photodefinition had so far been used only for fabricating passive waveguiding structures. Direct photo patterning of UV curable epoxy oligomers has already been demonstrated for the realization of low loss thermally stable multimode waveguides $[9,10]$. In this chapter the fabrication of EO microring resonators and monomodal bus waveguides by direct photodefinition is presented. The photodefinition technique is more advantageous in comparison with RIE which is widely being used for fabricating EO polymer waveguides.

1. As the structures are directly transferred from the mask to the wafer, dimensional accuracy is better than the RIE process involving an additional resist layer.

2. Photodefinition induces less side wall roughness compared with RIE resulting in lower scattering losses.

3. Especially in the case of MRs the side wall roughness created by RIE in the curved section is detrimental for the roundtrip losses, and photodefined rings have the clear advantage of reduced sidewall scattering.

4. In the case of laterally coupled polymer MRs the submicron gap size required between the ring and the bus waveguide cannot be achieved by RIE, at least not with our equipment.

5. When using RIE, material compatibilities between the photoresist and the core layer poses a serious problem. Also resist removal after etching becomes difficult especially when etching thick layers.

Despite these clear advantages, photodefinition has not been used for structuring EO polymers as most of the EO chromophores are absorbing in the UV region near the 365 $\mathrm{nm}$-I line. In that case long exposure times are required as the UV light is strongly absorbed in the upper film region before reaching the lower part. This long exposure times causes the chromophores at the surface to be bleached. The novel TCVDPA chromophore used in this work has a low absorption in the UV region around $365 \mathrm{~nm}$ (Fig. 3.2) allowing the host material to be photodefined. Another major issue in photodefining EO polymers is the alignment of the chromophores. Crosslinking of the host material is required for defining the structures and alignment of the chromophores (poling) is required to make the phodefined structures electro-optically active. Both the poling and crosslinking are thermally driven processes. Careful control of these two processes is required in order to achieve maximum poling efficiency which will be discussed in detail in this chapter. 


\subsubsection{Requirements of the photodefinable core material}

The material for photodefinition, which is similar to photoresists, should satisfy the following requirements. It must be easily soluble in spin coating solvents and must have high crosslinking efficiency upon light irradiation and adequate reactivity at the curing temperature. Low molecular mass gives rise to high crosslinking density and high density of crosslinking favors high resolution structures. This high degree of crosslinking induces shrinkage stress in the structure. Hence low shrinkage during crosslinking is necessary for dimensional accuracy and to avoid film stress. Good adhesion to the substrate is required. Most of the organic materials are hydrophobic and show poor wetting behavior to commonly used hydrophilic inorganic substrates like silicon or silicon oxide. Wetting can be improved by using commercial primers.

There are a number of commercially available photoresists for this kind of application. These materials are based on acrylate (free radical) crosslinking and form wet films after deposition. Photopatterning can only be done by using masks in the proximity mode which degrades the resolution. Moreover the acrylate crosslinking process requires an inert atmosphere to inhibit cure by ambient oxygen which adds to the production cost. Photocurable epoxies do not posses these drawbacks. The different (cationic) cure mechanism allows the processing of dry films with contact the mask under normal atmosphere.

SU8 is a well known negative photoresist invented by IBM [11-13] which can be photocured. This photoresist is commercially available from Microchem [14] and has been widely used in MEMS application as a negative tone photoresist because of high aspect ratio imaging with nearly vertical sidewalls. It is thermally stable with very good controllability of film thickness from 1 to several hundred microns [12, 15]. Up to now there are only a few reports on the fabrication of waveguides using this material $[16,17]$.

\subsection{SU8 negative resist as core material}

\subsubsection{SU8 processing}

The photodefinition process of SU8 is shown in Fig. 5.1. The polymer solution is spin coated onto the substrate and exposed through a contact mask to ultraviolet light. The UV light generates a photo-acid in the polymer. It is then thermally cured during which the photo-acid ring opens the epoxy groups and crosslinks them. The parameters which are to be controlled to get perfectly vertical side walls are the exposure time, curing temperature and curing time. As this process creates the negative of the structures on the mask, it is called a negative resist. 


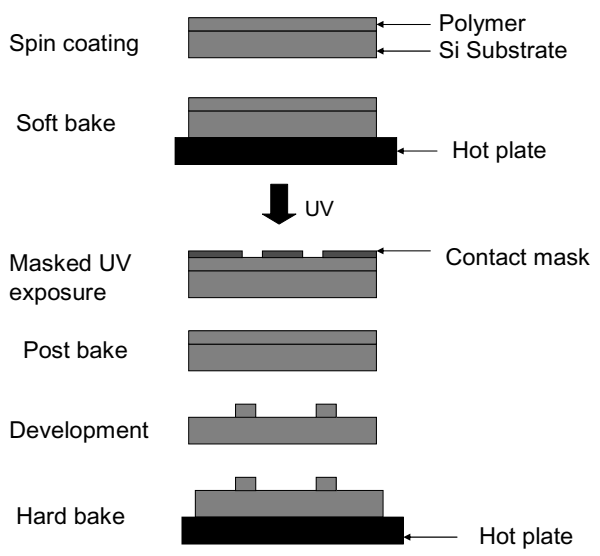

Figure 5.1: Photolithographic steps with the negative photoresist SU8.

\subsubsection{Chemical characteristics of SU8}

SU8 is a chemically amplified resist. It consists of acid-liable groups and a photoacid generator. Irradiation with UV light creates a low concentration of strong acid which will act as a catalyst of the crosslinking process of the epoxy. Subsequent heating of the resist activates crosslinking and regenerates the acid catalyst. As a consequence the sensitivity of the resist is significantly increased.

In particular, the main components of SU8 are Bisphenol A Novolak epoxy oligomers (Fig. 5.2) and $4 \mathrm{wt} \%$ triaraylsulfonium hexafluroantimonate salt (UVI) photoacid generator. Upon irradiation the photoacid generator decomposes to form hexafluroantimonic acid that protonates the epoxides on the oligomer. These protonated oxononium ions are available to react with neutral epoxides in a series of crosslinking reaction after application of heat. The chemical reactions involved in the crosslinking process are shown in Fig. 5.3 [18].

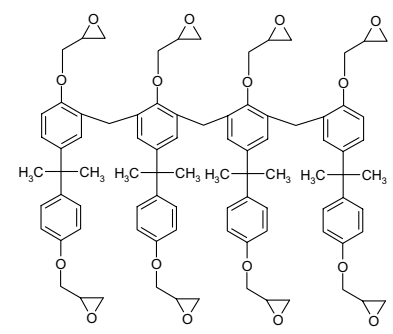

Figure 5.2: The chemical structure of the Bisphenol A Novalac epoxy oligomer contained in the SU8 formulation. 
Each monomer molecule contains eight reactive epoxy sites (Fig. 5.2) and therefore high degree of crosslinking can be obtained. This also results in a high mechanical and thermal stability after processing. Fully crosslinked SU8 has a glass transition temperature around $200^{\circ} \mathrm{C}$ and a degradation temperature $\sim 380^{\circ} \mathrm{C}$. The physical properties of SU8 are given in table 5.1 [18].

(b)

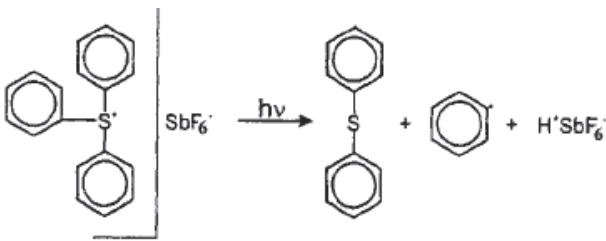

(c)

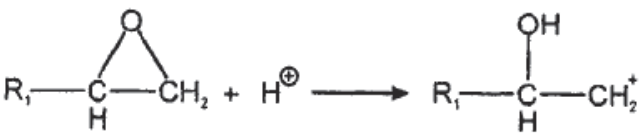<smiles>[R]CC1O[C+]1CC([R])O</smiles>

Figure 5.3: Crosslinking reaction in SU8. (b) Generation of acid upon UV exposure as the result of protolysis of triaraylsulfonium hexafluroantimonate, the cationic photoinitiator. (c) Initiation of the crosslinking process via opening of the epoxy group. (d) Chain propagation of the crosslinking process.

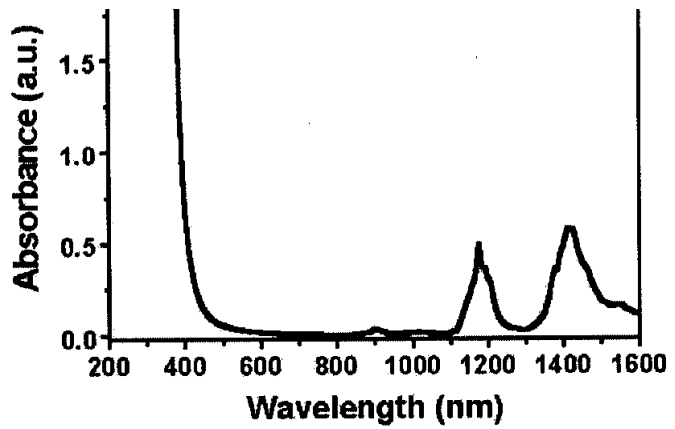

Figure 5.4: Absorption spectrum of fully crosslinked SU8. 
Table 5.1: Physical properties of SU8 photoresist.

\begin{tabular}{|c|c|c|}
\hline Property & $\begin{array}{c}\text { Processing } \\
\text { Parameter }\end{array}$ & Value \\
\hline Young's modulus & Post-bake at $95^{\circ} \mathrm{C}$ & $4,02 \mathrm{GPa}$ \\
\hline Young's modulus & Hard-bake at $200^{\circ} \mathrm{C}$ & $4,95 \pm 0,42 \mathrm{GPa}$ \\
\hline Biaxial modulus of elasticity & & $5,18 \pm 0,89 \mathrm{GPa}$ \\
\hline Film stress & Post-bake at $95^{\circ} \mathrm{C}$ & $16-19 \mathrm{MPa}$ \\
\hline Maximum stress & Hard-bake at $200^{\circ} \mathrm{C}$ & $34 \mathrm{MPa}$ \\
\hline Friction coefficient & Post-bake at $95^{\circ} \mathrm{C}$ & 0,19 \\
\hline Glasstransition temperature & Unexposed & $\sim 50{ }^{\circ} \mathrm{C}$ \\
\hline Glasstransition temperature & Fully cross-linked & $>200{ }^{\circ} \mathrm{C}$ \\
\hline Degradation temperature & Fully cross-linked & $\sim 380{ }^{\circ} \mathrm{C}$ \\
\hline Thermal expansion coefficient & Post-bake at $95^{\circ} \mathrm{C}$ & $52 \pm 5,1 \mathrm{ppm}$ \\
$\mathrm{K}^{-1}$ \\
\hline Polymer shrinkage upon cross-linking & & $7,5 \%$ \\
\hline
\end{tabular}

Fig. 5.4 shows the absorption spectrum of SU8. Low transparency in the UV/visible region is because of processing induced absorption (yellowing [10]) from photoinitiator decomposition products and polymer impurities [19]. The absorption tail due to yellowing increases the optical losses in the visible and near infrared region. Higher hard baking temperature above $200^{\circ} \mathrm{C}$ significantly increases absorption in the blue region. Hence the hard baking temperature is limited to $150^{\circ} \mathrm{C}$ for waveguide applications [16]. The $\mathrm{C}-\mathrm{H}$ and the $\mathrm{O}-\mathrm{H}$ peaks can also be seen in Fig 5.4. There exists very low absorption at $1.3 \mu \mathrm{m}$ but at $1.5 \mu \mathrm{m}$ the $\mathrm{O}-\mathrm{H}$ tail leads to increase in absorption. Slab waveguiding losses were reported to be $0.2 \mathrm{~dB} / \mathrm{cm}$ and $3 \mathrm{~dB} / \mathrm{cm}$ at $1.3 \mu \mathrm{m}$ and $1.5 \mu \mathrm{m}$ respectively [16].

\subsubsection{Commercially available SU8 formulations}

The first SU8 products were introduced commercially by Microchem in 1996. The resist is available in a wide range of formulations to cover thicknesses from $2 \mu \mathrm{m}$ to $300 \mu \mathrm{m}$ in a single coating process or to $3 \mathrm{~mm}$ by multiple coating processes [20]. The SU8 series is formulated in gamma-butyrolacton (GBL) as solvent. SU8 films with thicknesses below 2 $\mu \mathrm{m}$ can be obtained by diluting the standard formulation with GBL solvent. The spin curves of varying dilutions of the standard formulation SU8-25 (which gives $25 \mu \mathrm{m}$ thick film at $3000 \mathrm{rpm}$ ) with GBL are shown in Fig. 5.5. 


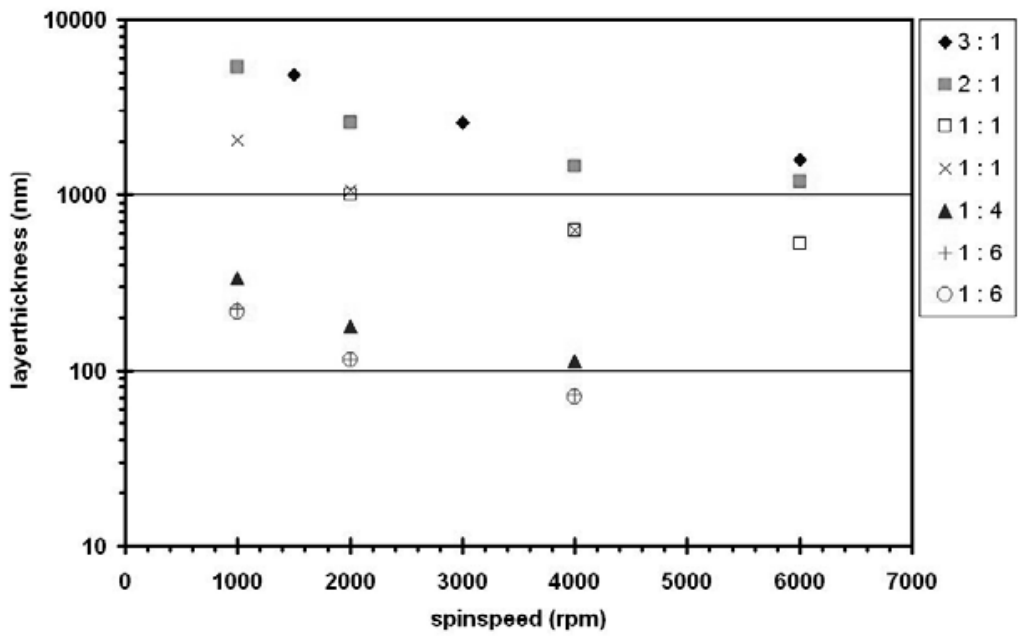

Figure 5.5: Spin curves of different dilutions of SU8-25 in GBL denoted as the weight ratio SU825:GBL [21].

Recently a new generation of SU8 resist, the SU8-2000 formulated with cyclopentanone as solvent with improved coating and adhesion properties has become commercially available.

\subsubsection{Lithography with SU8}

Processing of SU8 starts by selecting the adequate commercial variant suitable for the desired thickness. For film thickneses in the range of $1 \mu \mathrm{m}$ SU8-25 is diluted with GBL. This provides the possibility of mixing the chromophores separately in GBL for better solubility and filtering it. The GBL with chromophores can then be used to dilute SU8-25 to SU8-2.

Good wetting of the substrate by SU8 is crucial for obtaining homogeneous and stable coatings. The wetting properties are determined by the substrate surface energy. Being an organic material SU8 is rather hydrophobic (contact angle of cured resin is about $73^{\circ}$ ). Wetting can be improved by using adhesion promoters which are low molecular weight organic substances that either strongly adsorb or react with the inorganic surface forming a thin organic layer with low surface energy which can then be wet by SU8.

After the spin coating process the film is soft baked in order to remove the solvent and improve resist-substrate adhesion. This process is accompanied by shrinkage of the film. Soft baking is performed by heating the film at $95^{\circ} \mathrm{C}$ on a flat leveled hot plate. Higher soft baking temperatures $>120^{\circ} \mathrm{C}$ can initiate thermal crosslinking even before UV exposure [22]. Lower temperatures or short baking time leaves resist film with a high 
solvent content which will evaporate and therefore generate high film stress during post exposure baking. The residual solvent content in the film has also an influence on the refractive index of the final processed film. Solvents, if not completely removed, can result in the cracking of the top gold electrode during sputtering. Exposure is performed by masked irradiation with a UV lamp. The resist's UV absorption at these wavelengths causes a non uniform light intensity distribution through the thickness of the film, if the film thickness is too high. For a two micron film this effect was not seen and structures with perfectly vertical sidewalls can be obtained. After irradiation a post exposure bake initiates the crosslinking process in the irradiated areas and stabilizes them against dissolution in the subsequent development step. The exposure time is a critical parameter which needs to be optimized to achieve high resolution structures. Development is performed by immersing the substrate in propylene glycol methyl ether acetate (RER) at room temperature followed by a rinsing step in isoproponol. The developed structures are then subsequently hard baked at $150^{\circ} \mathrm{C}$.

\subsection{SU8-TCVDPA EO polymers}

SU8 can be doped with chromophores to form an electro-optic polymer. Two chromophore types were used namely TCVDPA and epoxy-TCVDPA with two epoxy groups attached to the donor side of the chromophore (Fig. 5.6). SU8-TCVDPA forms a guest-host polymer system in which the chromophore has no chemical linkage with the polymer. In this system the final $\mathrm{T}_{\mathrm{g}}$ of the material is reduced by the plasticizing effect of the chromophore and hence high concentration of the chromophore can only be attained at the cost of some $T_{g}$ reduction. By using TCVDPA with epoxy groups the chromophore can be attached to SU8 during the polymer crosslinking process. By this way the high $\mathrm{T}_{\mathrm{g}}$ attained in the case of fully crosslinked SU8 can be retained. Fig. 5.7 shows the absorption spectra of TCVDPA and epoxy-TCVDPA showing the low UV absorption window at the exposure wavelength of most mask aligners ( $\mathrm{I}, \mathrm{G}$ and $\mathrm{H}$ lines). It can also be seen that the $\lambda_{\max }$ of the chromophore is not shifted by the modification with the epoxy group. This low UV absorption window of the chromophore is exploited to photo-pattern the SU8 host material. This would not be possible with traditional chromophores like DR1 or DANS as very long exposure times will be required for homogeneous exposure of the host material. During such long exposure times the chromophores at the surface will be bleached.
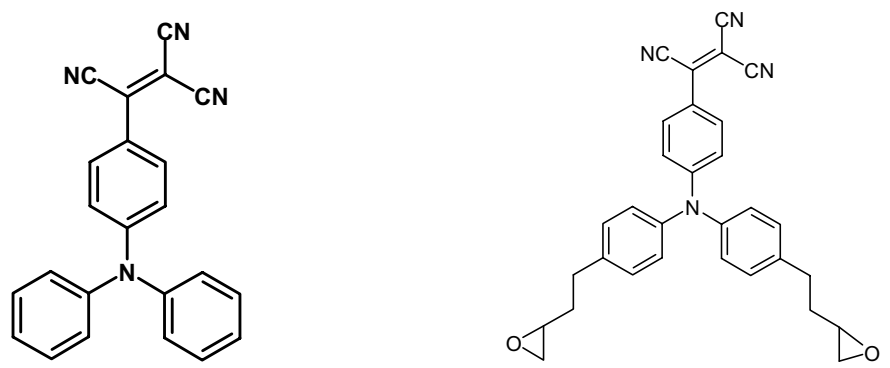

Figure 5.6: Chemical structure of TCVDPA (left) and epoxy-TCVDPA (right). 


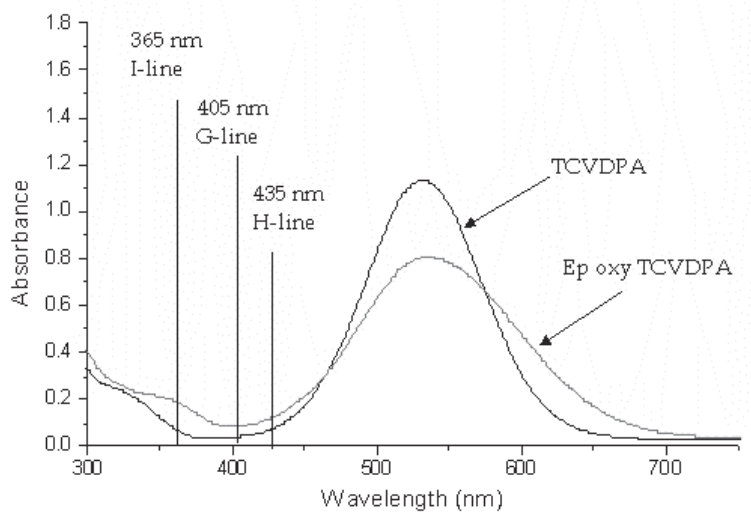

Figure: 5.7: Absorption spectra of TCVDPA and Epoxy-TCVDPA showing the low UV absorption window.

\subsubsection{Poling behavior of the SU8-TCVDPA EO polymer}

In the following section the poling of the SU8-TCVDPA system will be described in detail. Careful control of the poling and crosslinking processes will be required to achieve high poling efficiency. Poling has to be carried out together with the crosslinking process as poling is inhibited after complete crosslinking.

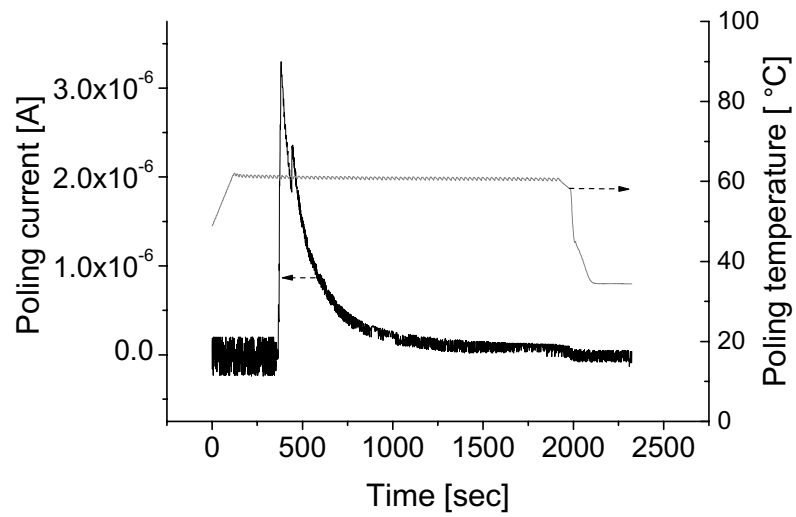

Figure 5.8: Poling of SU8-TCVDPA in the uncrosslinked state. 
The Poling behavior of SU8 containing $15 \mathrm{wt} \%$ TCVDPA and epoxy-TCVDPA was studied with films deposited on ITO coated glass substrates. A $100 \mathrm{~nm}$ gold layer was sputter deposited on the polymer to serve as poling electrode together with the ITO layer. The samples were then poled and the $\mathrm{r}_{33}$ coefficient was measured at $830 \mathrm{~nm}$. The electric field strength was $100 \mathrm{~V} / \mu \mathrm{m}$ in all cases.

First the poling of SU8-15wt\% TCVDPA was studied without allowing the starting of the crosslinking process. For this purpose poling was monitored by recording the poling current in an unexposed film. Fig. 5.8 shows the poling behavior poled at $50^{\circ} \mathrm{C}$ in the uncrosslinked state. The alignment of the chromophores after reaching $\mathrm{T}_{\mathrm{g}}$ is reflected in the high poling current when the poling field is switched on and the rapid exponential decay of the poling current at constant poling temperature and constant electric field. Once aligned the poling current is essentially zero. After poling no $\mathrm{r}_{33}$ could be measured as with the low $\mathrm{T}_{\mathrm{g}}$ of SU8 in the uncrosslinked state the chromophore relaxation is nearly instantaneous.

The effect of the crosslinking process on poling was studied by carrying out poling in SU8-15wt \% TCVDPA films which were flood exposed to UV light prior to poling. The UV dosage was varied and the chromophore alignment after different crosslinking degrees was studied.

Fig. 5.9 shows the poling behavior of a SU8-TCVDPA film which was highly exposed to UV at $365 \mathrm{~nm}\left(5 \mathrm{mins}\right.$ at $\left.12 \mathrm{~mW} / \mathrm{cm}^{2}\right)$ prior to poling. Crosslinking and poling go hand in hand. As poling proceeds the glass-transition temperature also raises and crosses the poling temperature. This highly impedes the poling process. For further poling to take place the poling temperature has to be raised. Poling could be increased by stepwise increasing the poling temperature.

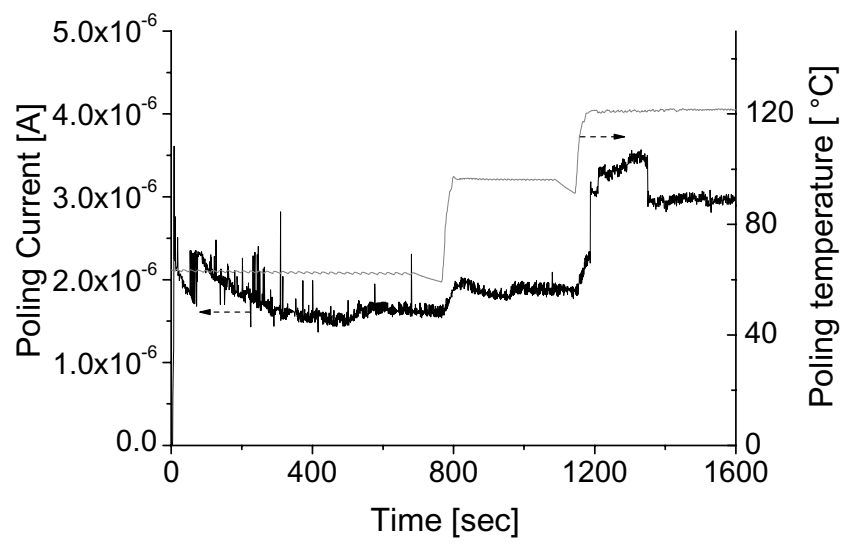

Figure 5.9: Poling behavior of SU8-TCVDPA after over exposure. 
At each temperature step a sharp rise and a slow decay in the current can be noticed indicating the occurrence of poling. The increase in current is mostly due to the increased conductivity of the polymer with higher temperature. This conduction current is constant in time and weakly dependent on the degree of crosslinking. After a short time after each temperature step the poling current is stopped as the crosslinking process dominates from there on. These two effects of poling and crosslinking are competing effects. Final poling induced order is determined by which of the two processes occurs fast. In the case of Fig. 5.9 no $r_{33}$ could be measured.

It would be ideal that crosslinking occurs just after the poling process. In this way the $\mathrm{T}_{\mathrm{g}}$ of the material could be raised during the course of chromophore alignment without affecting the poling process. With this aim poling was carried out with reduced exposure time of about $1 \mathrm{~min}$. Fig. 5.10 shows the resulting poling behavior of the SU8-TCVDPA system. Only slightly exposing the system to UV leads to slow cross linking so that there is enough time for the poling order to be set in. As can be seen in the figure, poling, which results in the exponential decay in the poling current proceeds until about 500 seconds. After this, the crosslinking process becomes dominant which is reflected in an almost linear decrease in current. After cooling down an $\mathrm{r}_{33}$ value of $1.2 \mathrm{pm} / \mathrm{V}$ was measured.

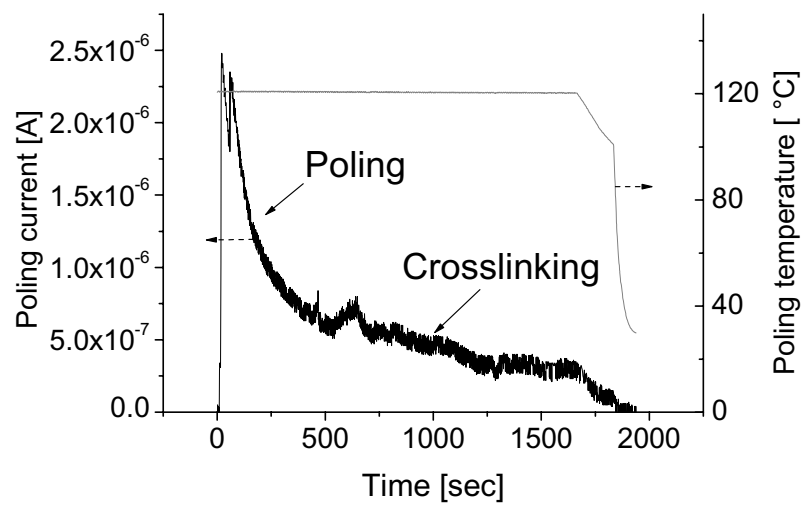

Figure 5.10: Poling behavior of a slightly UV exposed SU8-TCVDPA film.

In both the poling experiments shown in Fig. 5.7 and Fig. 5.8 the $\mathrm{T}_{\mathrm{g}}$ did not increase above $70^{\circ} \mathrm{C}$ after crosslinking.

DSC experiments were carried out to better understand the reasons of such a behavior. SU8 containing $1 \% \mathrm{wt} \%$ of thermal initiator was scanned between $50^{\circ} \mathrm{C}$ and $250^{\circ} \mathrm{C}$ at a scanning rate of $10^{\circ} \mathrm{C} / \mathrm{min}$. The graph in Fig. 5.11 shows a large exothermic band around $100^{\circ} \mathrm{C}$, that can be attributed to the cross-linking process of the SU8. Using similar experimental conditions a sample containing $25 \mathrm{wt} \%$ of epoxy-functionalized TCVDPA chromophore added to the SU8 matrix was scanned, but the resulting DSC curve shows 
no peak corresponding to the cross-linking process, probably due to the presence of the Ncontaining chromophore into the matrix that inhibits the completion of the polymerization, neutralizing the photoacid.

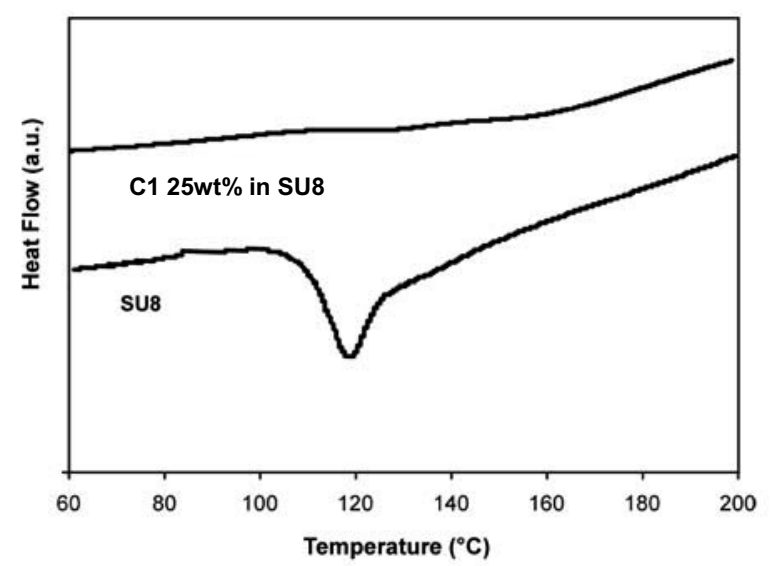

Figure 5.11: DSC curves of SU8 and SU8 with 25 wt\% -TCVDPA.

To support this hypothesis, as basic initiator imidazole was added to a $25 \%$ solution of TCVDPA in SU8 and cured by DSC at $100^{\circ} \mathrm{C}$ for $15 \mathrm{~min}$ and then scanned from $25^{\circ} \mathrm{C}$ to $300^{\circ} \mathrm{C}$. The resulting DSC curve shows a $\mathrm{C}_{\mathrm{p}}$ change at around $150^{\circ} \mathrm{C}$ that can be attributed to the $T_{g}$ of the resulting SU8-TCVDPA cross-linked polymer, demonstrating the ability to crosslink SU8-TCVDPA with basic initiators.

Since the thermal curing agent used in the DSC experiments, cannot be used in the UV cross-linking process, as alternative an extra amount of photoinitiator was added to compensate the neutralization induced by the chromophore.

Using an excess amount of acid initiator also seems to have the same effect on the $T_{\mathrm{g}}$. The exact amount of the extra photo acid required depends on the chromophore concentration. Fig. 5.12 shows the poling behavior of SU8-15 wt \% TCVDPA with $3 \mathrm{wt} \%$ extra photo-acid initiator (UVI- see section 4.3.1). The sample was UV exposed before poling for $1 \mathrm{~min}$. The poling voltage was first applied to the sample and the temperature was slowly raised from room temperature to $90^{\circ} \mathrm{C}$ in $30 \mathrm{mins}$ and maintained at $90^{\circ} \mathrm{C}$ for $5 \mathrm{mins}$. The discontinuity in the curve was caused by a experimental error during the experiment. Because of simultaneous poling and crosslinking the pure exponential behavior of the poling process is not observed. An $r_{33}$ of $5 \mathrm{pm} / \mathrm{V}$ was measured after poling. The glass transition was raised to $170^{\circ} \mathrm{C}$ after crosslinking and poling together. A similar kind of simultaneous poling and crosslinking experiment was carried out with SU8-15 wt\% epoxy-TCVDPA and an $r_{33}$ of $9 \mathrm{pm} / \mathrm{V}$ was measured with an increase in $\mathrm{T}_{\mathrm{g}}$ to $190^{\circ} \mathrm{C}$. 


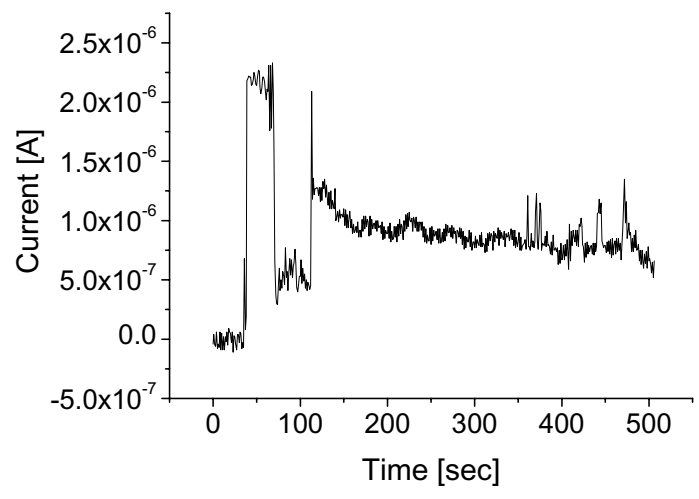

Figure 5.12: Poling behavior of SU8-15wt\% TCVDPA with 3wt\% extra photoacid. (poled and crosslinked simultaneously).

The poling recipe shown in Fig 5.12 yielded the best result. Thus it can be concluded that the highest poling efficiency can be attained in SU8 by performing carefully balanced simultaneous poling and crosslinking.

\subsection{Fabrication of microrings by photodefinition}

\subsubsection{Optimization of the lithographic process}

The photodefinition process of the microrings was tested in SU8-15 wt\%-TCVDPA. Lithography was carried out with a contact $\mathrm{Cr}$ mask. To the commercially available SU8 an extra $3 \mathrm{wt} \%$ photoacid generator was added to compensate the neutralization effect by the chromophores. The same mask described in chapter 4 was also used here. As the refractive index of SU8-15 wt\%-TCVDPA was not much different from PMMA-DR1 the same MR design was adopted also for SU8 rings.

Different exposure times varying from $30 \mathrm{sec}$ to 6 mins were applied. A threshold exposure time of at least $1 \mathrm{~min}$ was required for the waveguides to start appearing. Fig. 5.13 shows the ring section after development for exposure times from 1 to 4 minutes. As the exposure time increases, the width of the waveguides increases reducing the gap between the waveguides. A gap of about $500 \mathrm{~nm}$ was made possible (Fig. 5.14) with the exposure of 4 mins. For exposure times larger than this, the gap was not open. Fig 5.15 shows a SEM picture of a ring with an $800 \mathrm{~nm}$ gap. This approach offers the possibility to control the coupling constant by precisely adjusting the gap between the waveguide and the ring to be between $500 \mathrm{~nm}$ and $1 \mu \mathrm{m}$. This amount of fine tuning is not possible in waveguides obtained by RIE, which results in completely closed gaps when the mask had gaps below $1 \mu \mathrm{m}$. This restriction of RIE to large gap size results in much larger coupling sections and consequently increased effective ring radius, which is detrimental for the FSR 
and also increases the round trip loss of the ring. For the same coupling a $500 \mathrm{~nm}$ gap requires a coupling length of $40 \mu \mathrm{m}$ in contrast to $160 \mu \mathrm{m}$ for a gap of $1 \mu \mathrm{m}$.

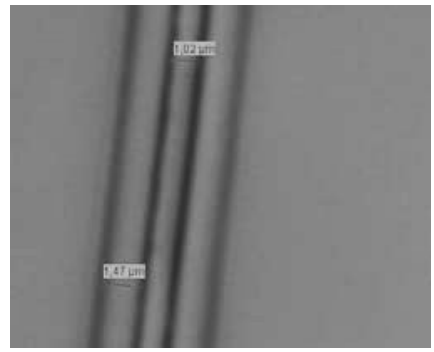

a

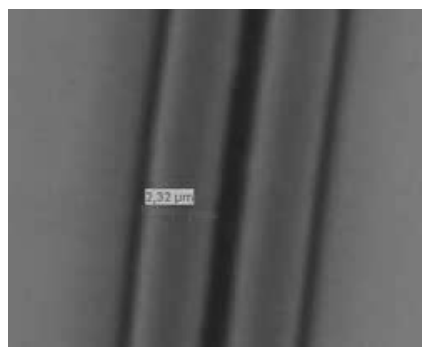

C

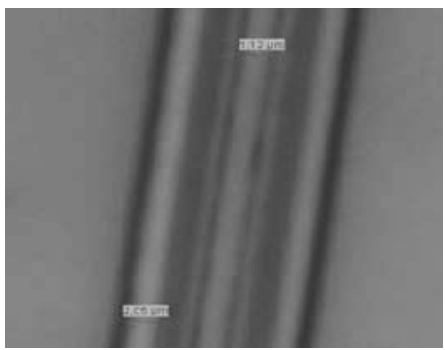

b

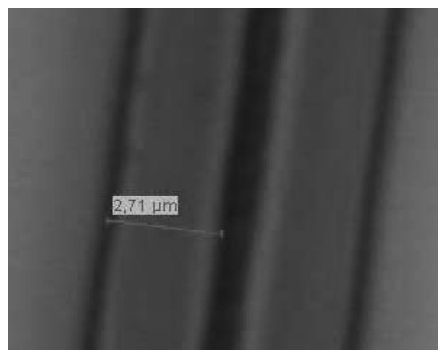

d

Figure 5.13: Effect of the exposure time on the development of the waveguide structure and the gap for different exposure times of 1 minute (a), 2 minutes (b), 3 minutes (c) and 4 minutes (d).

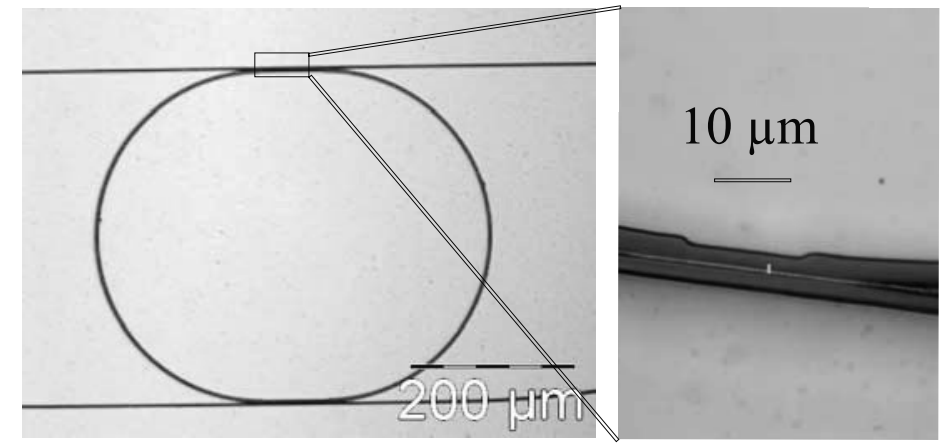

Figure 5.14: Microscope picture of a SU8-15 wt\% TCVDPA MR with a $500 \mathrm{~nm}$ gap. 


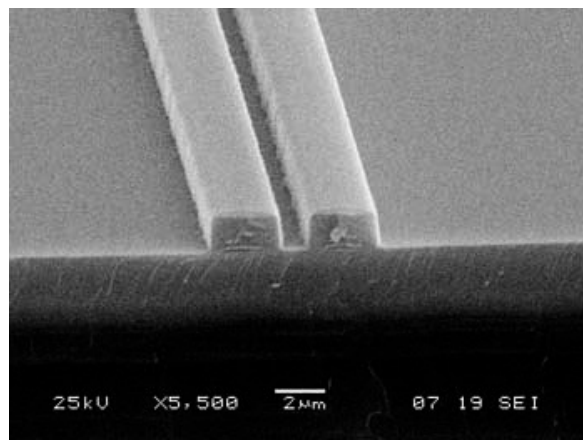

Figure 5.15: SEM picture of the coupling section of a microring with $800 \mathrm{~nm}$ gap.

\subsubsection{Device fabrication schemes}

\subsubsection{Fabrication by simultaneous poling and crosslinking (scheme 1)}

The fabrication of MRs by simultaneous poling and crosslinking, scheme 1, is shown in Fig. 5.16. In this case MRs with racetrack structures to increase the coupling between the straight waveguide and the ring were photodefined in SU8 $15 \mathrm{wt} \%$ TCVDPA containing 3 wt $\%$ extra photo acid.

The fabrication starts by the sputter deposition of a $250 \mathrm{~nm}$ gold layer on a silicon wafer. A $6 \mu \mathrm{m}$ layer of VSC was spin coated on top of the gold layer as bottom cladding and consequently UV exposed for $15 \mathrm{sec}\left(12 \mathrm{~mW} / \mathrm{cm}^{2}\right)$ and crosslinked at $95^{\circ} \mathrm{C}$ for 2 hours. Short exposure times and long curing times were applied to generate just sufficient photoacid for crosslinking. The bottom cladding was then exposed to ammonia atmosphere for 1 hour to neutralize any excess photo acid present which can diffuse into the core SU8 layer. SU8-25 (Microchem) diluted to SU8-2 containing $15 \mathrm{wt} \%$ of TCVDPA and $3 \mathrm{wt} \%$ additional photo-initiator (UVI) was spun on top of the bottom cladding. The film was exposed to UV for 4 mins $\left(12 \mathrm{~mW} / \mathrm{cm}^{2}\right)$ using a dark field mask with the ring design. A gold layer of $250 \mathrm{~nm}$ was sputtered on top of the exposed but still uncrosslinked SU8 film to serve as the top electrode during poling.

The poling voltage was applied to the film and the temperature was ramped from room temperature to $90^{\circ} \mathrm{C}$ in 30 mins. The sample was kept at $90^{\circ} \mathrm{C}$ for $5 \mathrm{mins}$ and cooled to room temperature in 15 mins. During this process the exposed regions got poled and crosslinked simultaneously. This method was tried with the idea of applying the maximum poling efficiency of simultaneous poling and crosslinking to the waveguide structure in the way as demonstrated before in the bulk film. 
It was found that the waveguides could not be fully developed in RER (SU8 developer [14]) after this process. Further investigation revealed that the film was exposed to excess UV as the unexposed regions were also crosslinked. This could have happened during the sputtering of the top poling electrode where the film is exposed to UV light from the argon plasma inside the sputterer. A similar unintended exposure was also encountered by applying vapor deposition of the top poling electrode as then the film was exposed to strong white light.

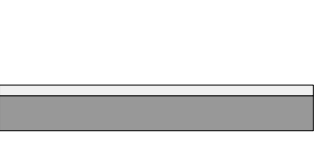

Sputtering $250 \mathrm{~nm}$ gold on Silicon substrate

Poled and crosslinked simultaneously

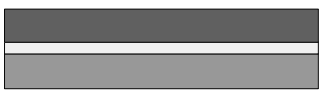

Bottom cladding (VSC) $6 \mu \mathrm{m}$ UV-15 sec. Hot plate $95^{\circ} \mathrm{C}$ for 30 mins.
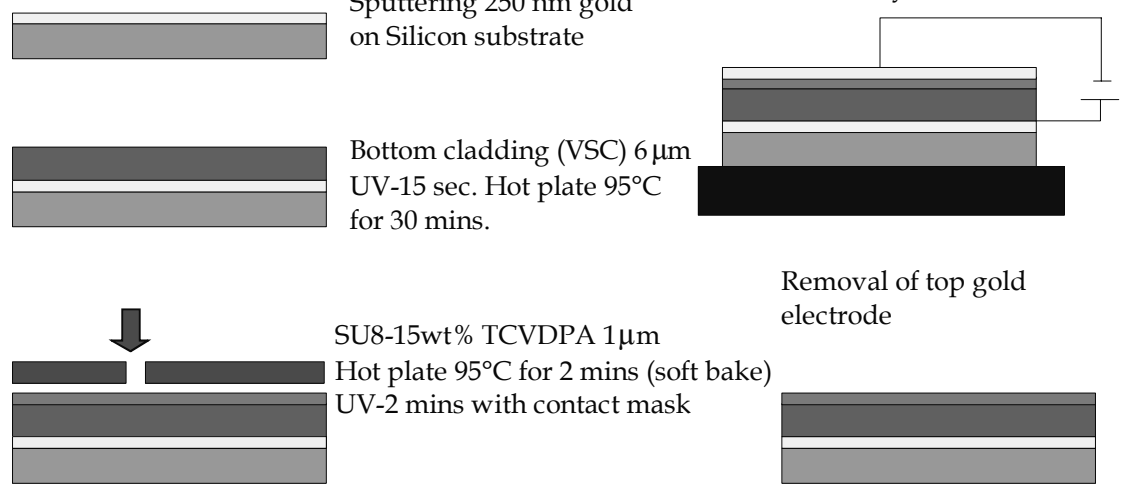

SU8-15wt \% TCVDPA $1 \mu \mathrm{m}$

Removal of top gold electrode Hot plate $95^{\circ} \mathrm{C}$ for 2 mins (soft bake) UV-2 mins with contact mask

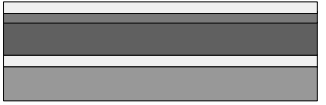

Sputtering $250 \mathrm{~nm}$ gold

Developed with RER

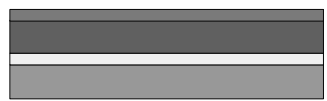

Figure 5.16: Fabrication steps for SU8-TCVDPA MRs involving simultaneous poling and crosslinking, scheme 1.

\subsubsection{Fabrication by initial partial crosslinking (scheme 2)}

In order to overcome the difficulty in defining the structure by scheme 1, an improved fabrication scheme was adopted (scheme 2). This involves partial crosslinking of the waveguides to define the structures, followed by simultaneous poling and further crosslinking as in scheme 1.

The fabrication steps for this scheme are shown in Fig. 5.17. The steps in scheme 1 are followed until exposing the SU8-TCVDPA layer. The film was then slightly crosslinked at $60^{\circ} \mathrm{C}$ for 15 mins and developed. A $6 \mu \mathrm{m}$ layer of top cladding (VSC) was spun on top. The top cladding was UV exposed for $15 \mathrm{sec}$ and crosslinked at $60^{\circ} \mathrm{C}$ for 5 mins. In this condition, the core layer and the top cladding are only slightly crosslinked. A top gold electrode was sputtered and patterned. The processing temperature of the wafer after exposure of the core SU8-TCVDPA layer was limited to $60^{\circ} \mathrm{C}$ to avoid further crosslinking of the core layer. Simultaneous poling and crosslinking was carried out as in scheme 1. 
Sputtering $250 \mathrm{~nm}$ gold on silicon

Bottom cladding (VSC) $6 \mu \mathrm{m}$ UV-15 sec. hot plate $95^{\circ} \mathrm{C}$ for 30 mins.

Su8-15wt\% TCVDPA $1 \mu \mathrm{m}$ Hot plate $95^{\circ} \mathrm{C}$ for 2 mins. (soft bake) UV- 2 mins. with contact mask

Partial crosslinking at $60^{\circ} \mathrm{C}$ for $5 \mathrm{mins}$
Developed with RER

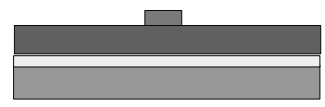

Top cladding (VSC) $6 \mu \mathrm{m}$

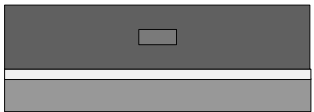

Sputtering of top electrode

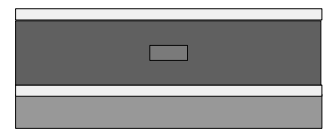

Poling and further crosslinking

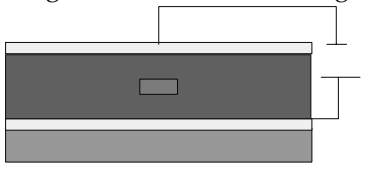

Figure 5.17: Process flow involving partial crosslinking (to define the MRs) and simultaneous poling and further crosslinking (to increase the $T_{8}$ ).

\subsubsection{Alternative fabrication method (scheme 3)}

It can be seen that by scheme 1 maximum poling efficiency can be achieved but resolution of the structures are severely affected. By scheme 2 MRs can be fabricated with high resolution but at the sacrifice of some poling efficiency because of the initial partial corsslinking. An alternative fabrication scheme to combine the poling efficiency of scheme 1 and the photdefinable properties of scheme 2 is suggested. This can be done by using a bright field mask and exposing the regions surrounding the waveguides first. The photoacid generated in the exposed region can then be neutralized by exposing the wafer to ammonia atmosphere. The waveguide region can then be given the required amount of UV dosage and simultaneous poling and crosslinking can be followed then on as in scheme 1. A further improvement in the poling efficiency of scheme 2 can be achieved by curing the bottom cladding also at $60^{\circ} \mathrm{C}$ so that during poling the resistivity of the core layer and the claddings will be almost the same.

\subsection{Experimental measurements}

\subsubsection{Measurement of the ring response}

The MR response from the rings fabricated by scheme 2 was studied by coupling in light from a broadband EDFA source emitting between $1530 \mathrm{~nm}$ and $1560 \mathrm{~nm}$. The light coming out of the through and drop port was analyzed with a spectrum analyzer. Fig. 5.18 shows the through and drop port response of a ring with a gap of $500 \mathrm{~nm}$ and a coupling section 
length of $40 \mu \mathrm{m}$. The through port response shows dips of $11 \mathrm{~dB}$ and at the drop port peaks of $4 \mathrm{~dB}$ were measured. The finesse of the ring was measured to be 6 and a ring waveguide loss of $25 \mathrm{~dB} / \mathrm{cm}$ and a field coupling constant of 0.8 were extracted by making a fit to the through port response using Rfit [23] software (Fig. 5.19).

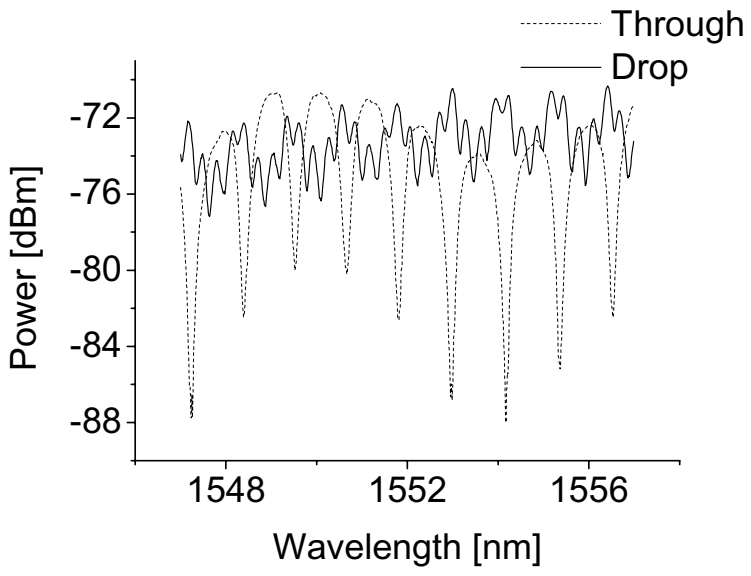

Figure 5.18: Through and drop port response of SU8-TCVDPA ring shown in Fig 5.14 for TM polarization.

The oscillations in the drop port response in Fig. 5.18 are because of the Fabry-Perot resonances of the straight section of the ring.

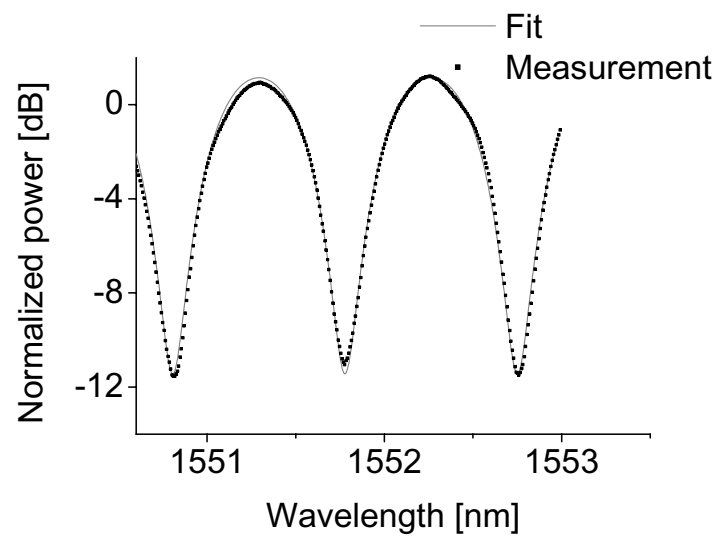

Figure 5.19: Through port response (dotted) and the fit made with Rfit software (line). 


\subsubsection{Analysis of the ring losses}

In order to understand the origin of the total ring losses, knowledge of the channel waveguiding loss and slab waveguiding loss is essential. Slab waveguiding loss (mostly absorption) of the core material was measured to be $1.6 \mathrm{~dB} / \mathrm{cm}$ at $1523 \mathrm{~nm}$. In order to asses the losses in the SU8-TCVDPA channel waveguide, quantitative image processing was made on pictures obtained with an IR camera (Fig. 5.21). For comparison first waveguides made by RIE of DR1 doped polymer waveguides were imaged (Fig. 5.20). These images were analyzed with curveloss software [24]. Fig. 22 shows the scatter intensity close to the incoupling facet when equal power is coupled to both waveguides. It appears that scattering from the photodefined waveguide is about four times less than that from the RIE waveguide. Channel waveguiding losses were measured by analyzing the scattering intensity as a function of the waveguide length and were found to be 5 $\mathrm{dB} / \mathrm{cm}$ for the photodefined waveguide and $16 \mathrm{~dB} / \mathrm{cm}$ for the RIE waveguide.

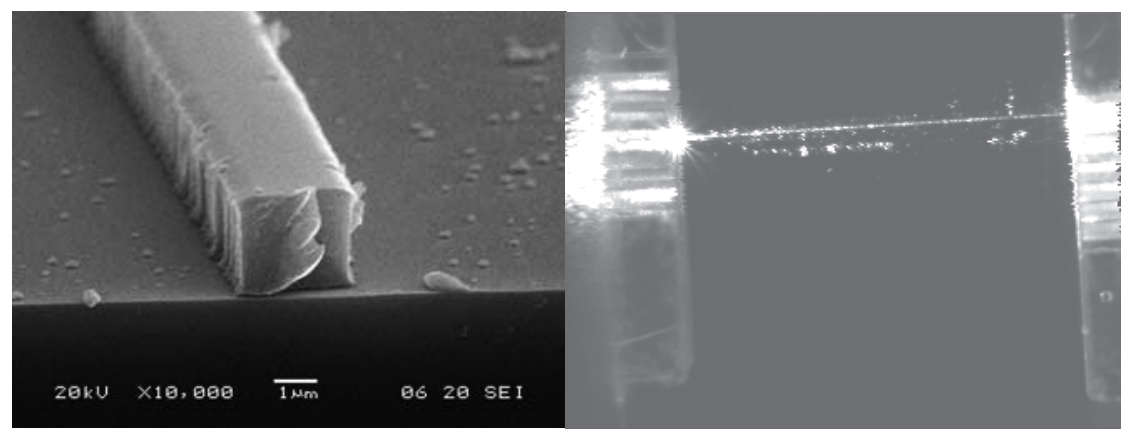

Figure 5.20: SEM image of a PMMA-DR1 waveguide made by RIE (left) and its infrared image with light at $1550 \mathrm{~nm}$ coupled in (right).

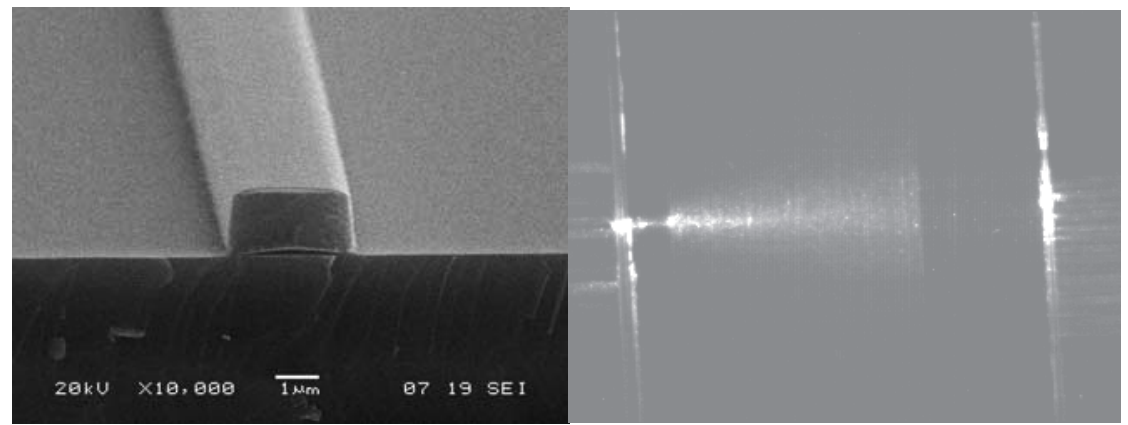

Figure 5.21: SEM image of SU8-TCVDPA channel made by photodefinition (left) and infrared camera image of it with $1550 \mathrm{~nm}$ light propagating through (right). 


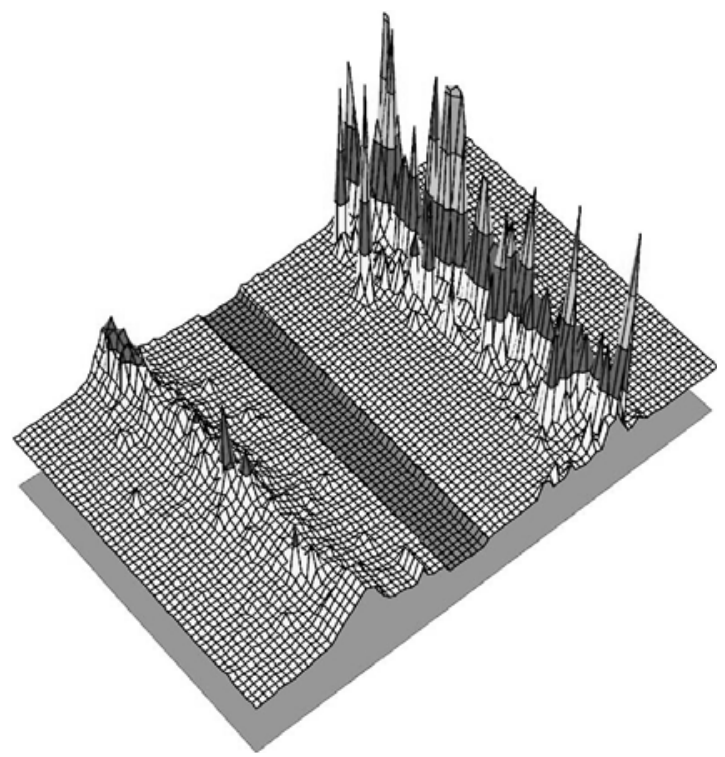

Figure 5.22: Intensity plot of the scattered light from waveguides with same input power: photodefined waveguide (left) and RIE waveguide (right).

The ring loss (total $25 \mathrm{~dB} / \mathrm{cm}$ ) has contributions from the bending losses (radiation 1 $\mathrm{dB} / \mathrm{cm})$, channel waveguiding losses $(5 \mathrm{~dB} / \mathrm{cm})$, and losses because of modal mismatch $(0.1 \mathrm{~dB} /$ transition $)$ at the interface between the curved and the straight section of the ring. For a finesse of 6 the modal mismatch losses would be about $7 \mathrm{~dB} / \mathrm{cm}$. This means that the ring scattering losses would be about $12 \mathrm{~dB} / \mathrm{cm}$ which is much higher than the straight channel waveguide losses of $5 \mathrm{~dB} / \mathrm{cm}$. The ring section has slightly higher side wall roughness compared with the straight section. This fact combined with the reason that the ring mode is shifted more to the outside of the ring making the intensity at the corecladding interface very high can give rise to high scattering losses.

\subsubsection{Measurement of EO modulation}

EO modulation was measured at the through port of the ring shown in Fig. 5.14 fabricated using scheme 2. Laser light from a tunable laser (around $1550 \mathrm{~nm}$ ) was butt coupled to the MR device using a polarization maintaining fiber (PMF). The polarization state of the light was controlled using a polarization controller between the laser and the PMF. The light was out-coupled from the through port of the device with a standard single mode fiber (SMF) and detected using a InGaAs high speed detector. The detector signal was monitored with an oscilloscope. The schematic picture of the experimental set-up is shown in Fig. 5.23. The modulation voltage from a function generator was amplified and applied on the MR using RF probes. 


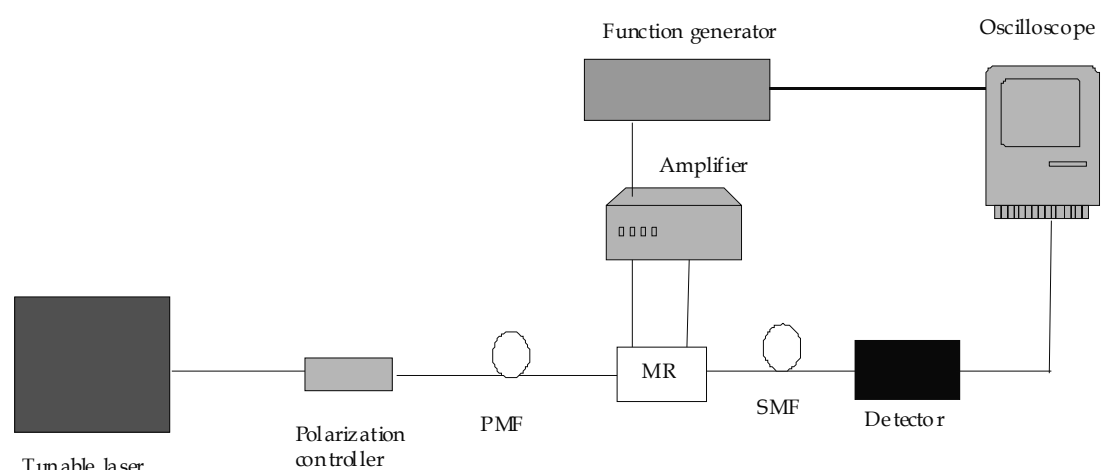

Figure 5.23: Schematic of the EO modulation measurement set-up.

The laser wavelength was tuned to be at $1550 \mathrm{~nm}$. A modulation signal of $200 \mathrm{~V}$ at $1 \mathrm{KHz}$ was applied between the top and the bottom electrode and the output at the through port was recorded using an oscilloscope. The measured EO modulation at the through port is shown in Fig. 5.24. The EO modulation amplitude was $20 \mathrm{mV}$.

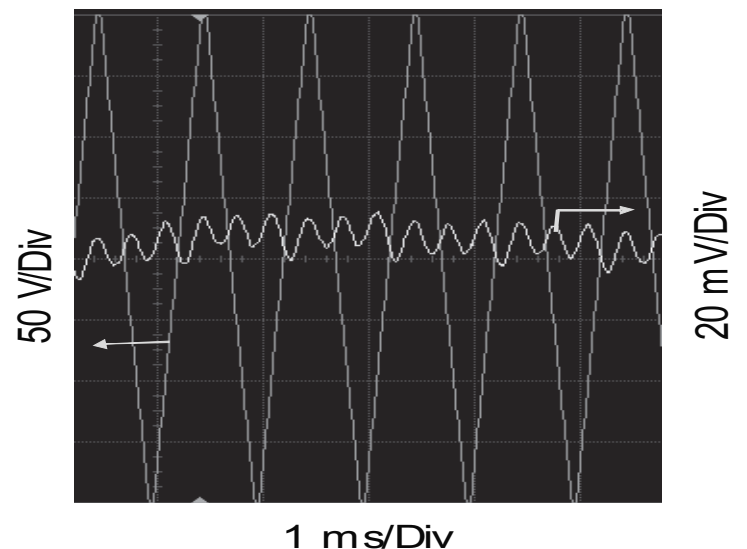

Figure 5.24: $1 \mathrm{KHz}$ EO modulation from SU8-TCVDPA MR.

In Fig. 5.24 the through-port spectrum has been moved by over a complete FSR. The plot of output amplitude as a function of the applied voltage is shown in Fig. 5.25. 


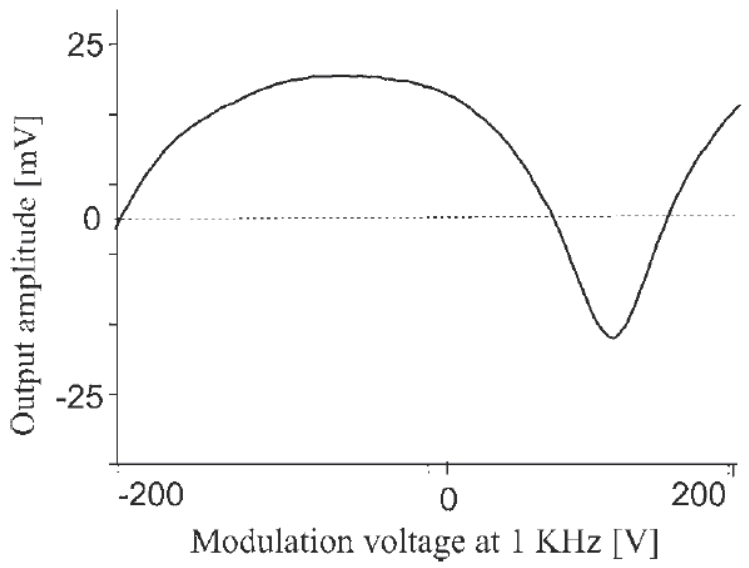

Figure 5.25: Output amplitude at the through port as a function of applied voltage on the electrodes.

Fig. 5.26 shows the EO modulation observed by tuning the laser wavelength to be at the maximum slope of the through-port response. The applied voltage in this case was $50 \mathrm{~V}$.

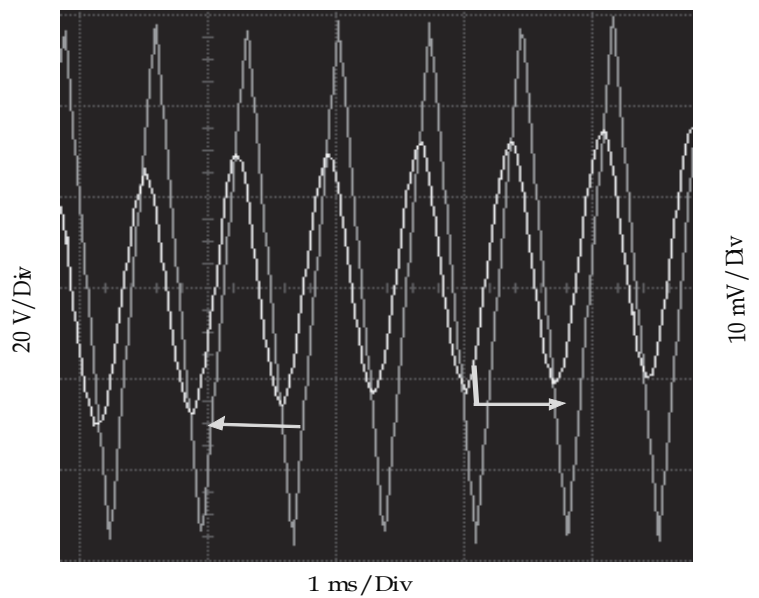

Figure 5.26: $1 \mathrm{KHz}$ EO modulation of SU8-TCVDPA MR with the wavelength of the light tuned to be at the maximum slope of the through port response.

The index change $\Delta n_{e}$ required to shift the resonance by $\Delta v_{F W H M}$ is 


$$
\frac{\Delta n_{e}}{n_{e}}=\frac{\Delta v_{F W H M}}{v}
$$

where $n_{e}$ is the effective index of the MR and $U$ is the optical frequency [25]. From this equation $\Delta n_{e}$ was calculated to be $2.10^{-4}$. The index change of the EO materials is given by

$$
\Delta n_{e}=\frac{1}{2} K n^{3} r_{33} \frac{V}{d}
$$

Where $K$ is the confinement factor, $V$ is the applied voltage, $\mathrm{n}$ is the refractive index of the EO material and $d$ is the distance between the electrodes. Assuming that the electric field is uniformly distributed in the stack and for a confinement factor of $0.7, r_{33}$ is estimated to be $10.6 \mathrm{pm} / \mathrm{V}$.

Fig. 5.27 shows the EO modulation at $10 \mathrm{MHz}$ on the same MR.

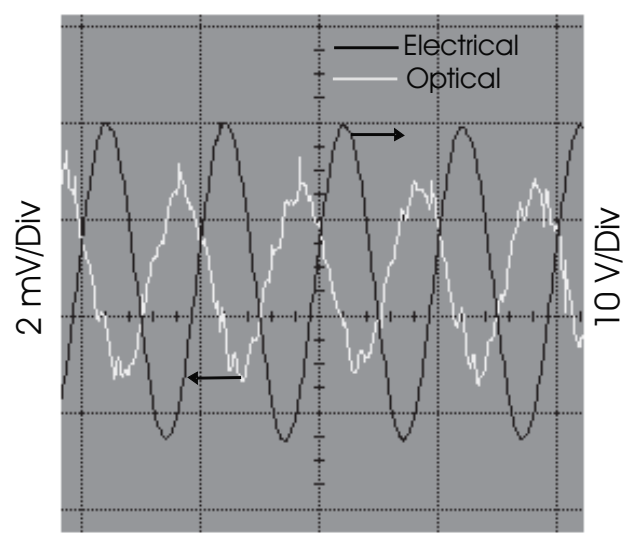

Figure 5.27: $10 \mathrm{MHz}$ EO modulation of SU8-TCVDPA MR.

The $r_{33}$ value calculated from Fig. 5.27 also yields a value of about $10 \mathrm{pm} / \mathrm{V}$.

The device should be further optimized to reduce the driving voltage. This can be done by

1. Reducing the thickness of the cladding layers, which was intentionally made thick in the current devices. 
2. Increasing the chromophore concentration using crosslinkable epoxy-TCVDPA.

The device has an extremely high photostability even without any packaging or shielding to oxygen. As a test light form a tunable laser source at $1550 \mathrm{~nm}$ was coupled to the device. With an insertion loss of $20 \mathrm{~dB}$ and a laser power of $15 \mathrm{~mW}$, power coupled into the waveguide is estimated to be $1.5 \mathrm{~mW}$. After about $100 \mathrm{~h}$, no reduction in $r_{33}$ was observed.

\subsubsection{Epoxy-gold reactions}

Delamination of the devices (Fig. 5.28) from the substrate prevented further measurements to be made at higher frequencies. The problem stems from the photoinitiator for the epoxy [26]. This is a sulfur containing salt. The sulfur has a high affinity for gold. As a result, the photodecomposition products concentrate at the gold interface and form a so-called weak boundary layer because they do not react with the epoxy. As the accumulated compounds are ionic in nature, there will be a high affinity for water with decrease moisture resistance of the joint. A cure for the problem might be a covering of the gold by a non-reactive metal, or the application of a sulfur containing adhesion promoter to the gold first, before the application of the epoxy. A thermal cure of the bottom cladding can also provide a solution.

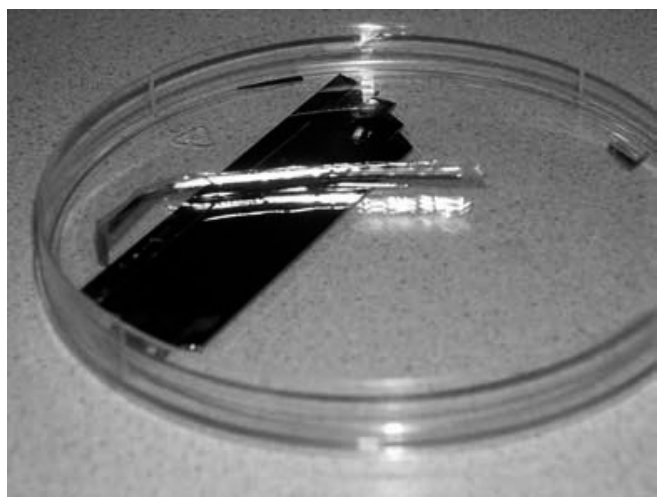

Figure 5.28: Picture of delaminated layer stack from the bottom gold electrode.

\subsection{Conclusions}

Photodefinition of microring resonators were done with SU8 as the host material, exploiting the low UV absorption window of the TCVDPA chromophore. Poling of such a 
system is generally very difficult because of the presence of highly conducting species in the photoinitiator. Poling (of the chromophore) and crosslinking (of the host) were optimized to achieve maximum $r_{33}$ value. The effect of neutralization of the photoacid by TCVDPA was compensated by an excess of photoacid to the commercially available composition of SU8. Poling experiments failed when the film is either completely uncrosslinked or was very poor in a completely crosslinked film. Best $r_{33}$ values were measured by carrying out poling and crosslinking simultaneously by increasing the temperature in a controlled manner. An $\mathrm{r}_{33}$ of about $6 \mathrm{pm} / \mathrm{V}$ was measured with $15 \mathrm{w} \%$ C1 in SU8 which is comparable with values obtained in Polysulfone guest-host system. Microring resonators were made by initially crosslinking an SU8-15 w\% TCVDPA film at $60^{\circ} \mathrm{C}$ to define the structures. A gap of $500 \mathrm{~nm}$ was possible. Channel waveguiding losses were measured to be $5 \mathrm{~dB} / \mathrm{cm}$. This is an significant improvement compared with 16 $\mathrm{dB} / \mathrm{cm}$ channel losses in a waveguide made by RIE. The microrings showed a throughport dip of $11 \mathrm{~dB}$ and the ring losses were $25 \mathrm{~dB} / \mathrm{cm}$. The EO modulation in these rings was demonstrated at $10 \mathrm{MHz}$ and an in in-device $\mathrm{r}_{33}$ of $11 \mathrm{pm} / \mathrm{V}$.

\subsection{References}

[1] J. W. Kang, J. P. Kim, W. Y. Lee, J. S. Kim, J. S. Lee, J. J. Kim, J. Lightwave Tech., 19, $p 872,2001$.

[2] J. R. Kulisch, H. Franke, R. Irmscher, C. Buchanl, Appl. Phys, 71, p3123, 1992.

[3] A Mukherjee, B. J. Eapen, S. K. Barla, Appl. Phys. Lett., 65, p3179, 1994.

[4] W. H. Wong, J. Zhou, E. Y. B. Pun, Appl. Phys. Lett., 78, p2110, 2001.

[5] A. Neyer, T. Knoche, L. Muller, Electron. Lett, 29, p399, 1993.

[6] Y. Shi, W. H. Steir, L. Yu, M. Chen, L. R. Dalton, Appl. Phys. Lett., 58, p1131, 1991.

[7] I. Assaid, I. Hardy, D. Bose, Opt. Comm., 214, p171, 2002.

[8] J. W. Kang, J. J. Kim, J. Kim, X. Li, M. H. Lee, IEEE Photon. Technol. Lett., 14, p1297, 2002.

[9] K. Enbustu, M. Hikita, R. Yoshimura, S. Tomaru, S. Imamura, Nonlinear Opt., 22, p441, 1999.

[10] M.B.J. Diemeer, L. Hilderink, R. Dekker, A. Driessen, IEEE Photon. Technol. Lett., 18, $p 1624,2006$.

[11] N. LaBianca, J. D. Gelorme, Proc. SPIE, 2438, p846, 1995.

[12] H. Lorenz, M. Despont, N. Fahrni, N. LaBianca, P. Renaud, P. Vettiger, Micromech. Microeng., 7, p121, 1997. 
[13] M. Despont, H. Lorenz, N. Fahrni, J. Brugger, P. Renaud, P. Vettiger, MEMS'97, p518, 1997.

[14] $\quad$ www.microchem.com

[15] B. Eyre, J. Blosiu, D. Wiberg, MEMS'98, p218, 1998.

[16] J. S. Kim, J. W. Kang, J. J. Kim, Jpn.J. Appl. Phys. 42, p1277, 2003.

[17] A Borreman, S. Musa, A. A. M. Kok, M. B. J. Diemeer, A. Driessen, IEEE/LEOS Benelux Chapter, p83, 2002.

[18] W. H. Teh, u. Durig, U. Drechsler, C. G. Smith, H. J. Guntherodt, J. Appl. Phys., 97, p054907, 2005.

[19] J. S. Kim, J. W. Kang, J. J. Kim, J. Appl. Phys., 42, p1277, 2003.

[20] H. Lorenz, MEMS'98 Sensors Actuators, 64, p33, 1998.

[21] A. Leinse, Integrated Optical Microsystems, PhD thesis, University of Twente, Enschede, The Netherlands.

[22] B. H. Ong, Opt. Lett., 31, p1367, 2006.

[23] E. J. Klein, Non-commercial microring fit software, Integrated Optical Microsystems, University of Twente, The Netherlands.

[24] M. Hoekman, Non-commercial Image processing software, Integrated Optical Microsystems, University of Twente, The Netherlands.

[25] P. Rabiei, W. H. Steier, C. Zhang, L. R. Dalton, J. Lightwave. Tech., 20, p1968, 2002.

[26] A. Hartwig, J. Adhesion Sci. Tech., 17, p1561, 2003. 


\section{NLO Polycarbonates}

In this chapter the functionalization of polycarbonates (PC) with TCVDPA chromophores is presented. This approach opens the way for a new class of NLO polymers combining the high photostability of TCVDPA and high Tg of polycarbonate. Different ways of functionalization of PC with TCVDPA are presented. The NLO coefficient $r_{33}$ of poled PC-TCVDPA were measured and the relaxation of the poling order was studied. With our results it is

demonstrated that it is possible to functionalize PC with high $\mu \beta$ chromophores.

Accepted for publication: M. Faccini, M. Balakrishnan et al, Journal of materials chemistry, 18. pp.2141-2149, 2008. 


\subsection{Introduction}

In the past decade, a large number of NLO chromophores has been synthesized and incorporated as a guest in a wide variety of host polymers [1- 4]. Nonlinear optical polymers should have a high $\mathrm{T}_{\mathrm{g}}$ to have long term stability of the poling order. Unfortunately stiff and extremely high $\mathrm{T}_{\mathrm{g}}$ polymers such as polyimides exhibit poor solubility in traditional spin-coating solvents. High $\mu \beta$ chromophores cannot survive the harsh solvents required for polyimides. Moreover, the high poling temperature required by the high $\mathrm{T}_{\mathrm{g}}$ polyimides can result in problems with chromophore sublimation when guest-host materials are used. On the other hand low $T_{g}$ polymers such as PMMA, which yields good optical quality films and good poling efficiencies do not yield adequate stability of poling-induced order. A compromise can be reached by using moderately high $\mathrm{T}_{\mathrm{g}}$ polymers like polycarbonate. The currently most investigated materials for this approach are various polyquinones [5-7] and polycarbonate (PC). This chapter demonstrates the possibility of functionalization of polycarbonate with different NLO chromophores and its usage as electro-optic material.

PC has been extensively investigated as a host polymer, due to its low crystallization tendency, good solubility in spin-casting solvents and high glass transition temperature $\left(T_{g}=205^{\circ} \mathrm{C}\right)[4,8]$. Moreover, its compatibility with large $\mu \beta$ chromophores and its high dielectric constant allow a good poling efficiency [9]. Although the $\mathrm{T}_{\mathrm{g}}$ of PC polymers is high, the incorporation of a chromophore will induce plasticization, considerably lowering the $\mathrm{T}_{\mathrm{g}}$ of the composite material, and therefore reducing the temporal stability of the poling induced alignment [10]. One way to solve these problems is by covalently incorporating the chromophores into high- $\mathrm{T}_{\mathrm{g}}$ polymers [11]. Surprisingly, in spite of the exceptional properties of polycarbonates when employed as host, there are only a few reports about the incorporation of chromophores as a side-chain in a polycarbonate backbone [12]. This chapter presents the optical properties of PC functionalized with TCVDPA and other traditionally used chromophores.

To provide EO materials with improved stability, the TCVDPA chromophore is incorporated into the polycarbonate backbone. The properties of six novel thermally and photochemically stable NLO polycarbonates is described. In these the TCVDPA chromophore is double end linked (14-A, 14-Z in Fig. 6.1), single end linked (16-A, 16-Z in Fig. 6.1) or double-end linked with a spacer (15-A, 15-Z in Fig. 6.1) into the polymer backbone. Moreover, the effect of the attachment mode and the flexibility on the poling efficiency and alignment stability is studied. Also a comparison is made with a guest-host system incorporating the same chromophore. 

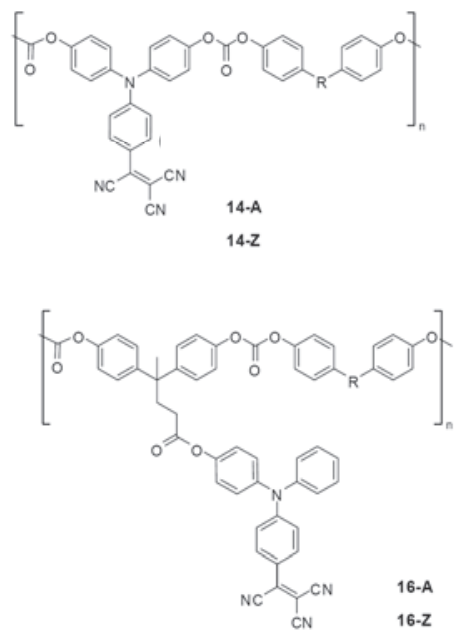
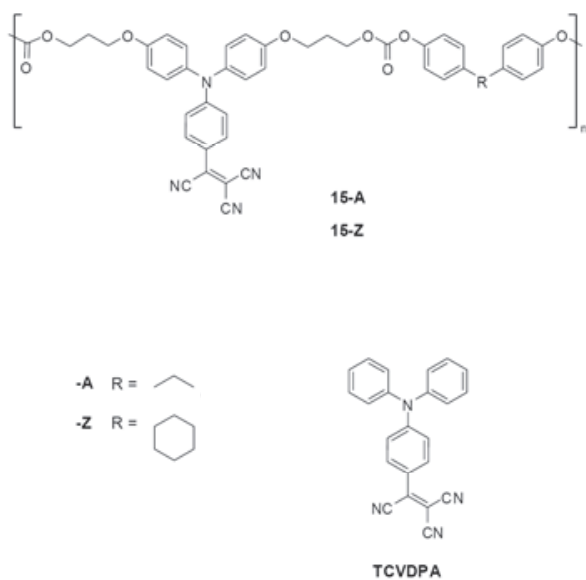

TCVDPA

Figure 6.1: Different ways of functionalization of TCVDPA in low $T_{g}(A)$ and high $T_{g}(Z)$ versions of $P C$.

\subsection{Functionalization of PC with TCVDPA}

In Chapter 3 we have described a series of chromophores based on the highly thermal and photostable TCVDPA $[13,14]$ which were incorporated as a guest at high loading in polysulfone. Although the increase of the chromophore concentration led to higher EO coefficients, it also resulted in a dramatic decrease of the $\mathrm{T}_{\mathrm{g}}$ of the polymeric material. To avoid this a different approach is chosen. As shown in Fig. 6.1, instead of preparing a guest-host system polycarbonate is now functionalized with TCVDPA in several variations. For this the high $\mathrm{T}_{\mathrm{g}}$ (bisphenol $\mathrm{Z}$ ) and low $\mathrm{T}_{\mathrm{g}}$ (bisphenol $\mathrm{A}$ ) version of the polycarbonate were used.

Polymer 14 acts like a main chain polymer and polymer 16 as a side chain polymer. Main chain polymers have restricted mobility of the chromophore but will have high thermal stability. Side chain polymers allow more chromophore movement than the main chain polymers but at the same time the chromophore alignment will also relax more easily when the poling field is removed. Polymer 15 is the same as 14 with some more back bone flexibility. Table 6.1 summarizes the physical and the optical properties of these polymers. 
Table 6.1: Summary of the thermal, physical, and optical properties of the NLO polycarbonates.

\begin{tabular}{|c|c|c|c|c|c|c|c|}
\hline & $\begin{array}{l}\lambda_{\max }^{b} \\
(\mathrm{~nm})\end{array}$ & $\begin{array}{l}T_{d}{ }^{c} \\
\left({ }^{\circ} \mathrm{C}\right)\end{array}$ & $\begin{array}{l}T_{g} \\
\left({ }^{\circ} \mathrm{C}\right)\end{array}$ & $M_{\mathrm{w}}$ & $M_{w} / M_{n}$ & $\begin{array}{l}\text { Chrom. } \\
\text { content } \\
(w t \%)\end{array}$ & $\begin{array}{l}r_{33} \\
(\mathrm{pm} / \mathrm{V})^{d}\end{array}$ \\
\hline TCVDPA & 531 & 360 & 85 & - & - & 35 in PS $^{e}$ & 12 \\
\hline 14-A & 523 & 371 & 206 & 12700 & 2.3 & 39.8 & 15 \\
\hline $14-Z$ & 523 & 380 & 215 & 14600 & 1.9 & 38,6 & 15 \\
\hline 15-A & 524 & 351 & 166 & 12400 & 1.7 & 37.0 & 33 \\
\hline $15-Z$ & 524 & 359 & 172 & 8300 & 1.9 & 36.2 & 31 \\
\hline $16-A$ & 526 & 329 & 178 & 12400 & 1.8 & 35.3 & 13 \\
\hline $16-Z$ & 526 & 341 & 184 & 10900 & 1.8 & 33.1 & 12 \\
\hline
\end{tabular}

${ }^{\mathrm{b}} \lambda_{\max }$ was measured in $\mathrm{CH}_{2} \mathrm{Cl}_{2} ;{ }^{\mathrm{c}}$ the onset temperature of degradation ( $\mathrm{T}_{d}$ onset)-see Table $3.2^{\mathrm{d}}$ measured at $830 \mathrm{~nm} ;{ }^{\mathrm{e}}$ as guest in polysulfone host (data taken from Chapter 3 ).

The decomposition temperature $\left(\mathrm{T}_{\mathrm{d}}\right)$ of all choromophore-containing polycarbonates is in the range between $329^{\circ} \mathrm{C}$ and $380^{\circ} \mathrm{C}$, whereby the double end choromophore linked polycarbonates $14-\mathrm{A}$ and $14-\mathrm{Z}$ appear to be the most stable structures. Due to their more rigid structure, these two polymers, in spite of the high chromophore loading of over 38 wt $\%$, possess the highest $\mathrm{T}_{\mathrm{g}}$ of the whole series, being $206^{\circ} \mathrm{C}$ and $215^{\circ} \mathrm{C}$, respectively. This result represents a significant improvement of over $100^{\circ} \mathrm{C}$ of the $\mathrm{T}_{\mathrm{g}}$ compared with guesthost systems incorporating the TCVDPA chromophore at similar high loadings (Chapter 3 ), and about $80^{\circ} \mathrm{C}$ over PMMA-DR1 side chain polymers (Chapter 4).

While the introduction of flexible spacers can enhance the chromophore mobility, facilitating the poling process, it is known that it will reduce the $T_{g}$ of the material [15]. For the $16-\mathrm{A}$ and $16-\mathrm{Z}$ polycarbonates, having the chromophore anchored as side-chain, the $\mathrm{T}_{\mathrm{g}}$ drops to $178^{\circ} \mathrm{C}$ and $184^{\circ} \mathrm{C}$, respectively. However, this drop is even more pronounced when three- spacers are introduced between the chromophore and the bisphenol moieties, leading to a $\mathrm{T}_{\mathrm{g}}$ of $166^{\circ} \mathrm{C}$ for polymer $15-\mathrm{A}$ and $172^{\circ} \mathrm{C}$ for polymer $15-\mathrm{Z}$. The higher intrinsic structural rigidity induced by the cyclohexyl group [16] in the bisphenol $\mathrm{Z}$ monomer, generally results in polymers with substantially higher $\mathrm{T}_{\mathrm{g}}\left(6^{\circ} \mathrm{C}\right.$ to $9^{\circ} \mathrm{C}$ increment $)$ with respect to those containing a bisphenol A monomer, having two methyl groups instead. 


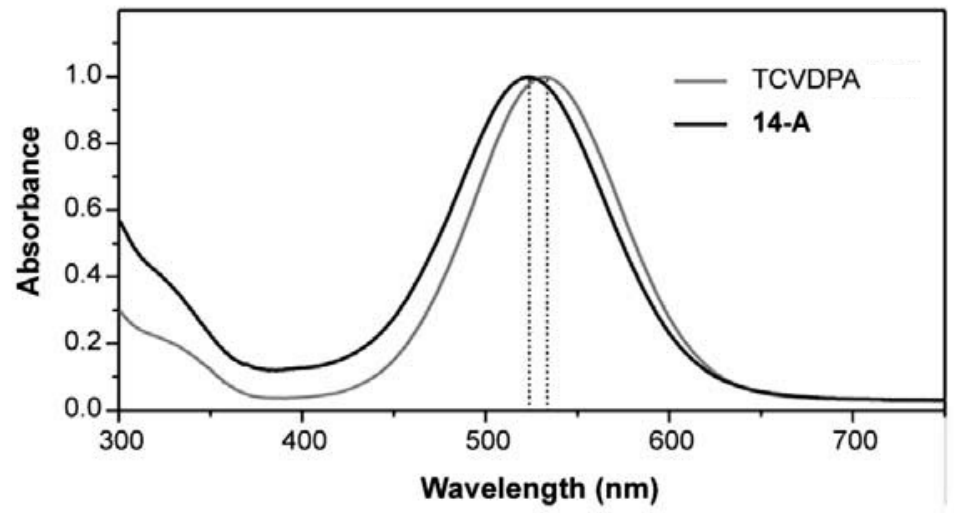

Figure 6.2: Absorption spectrum of the TCVDPA chromophore and the 14-A polymer dissolved in $\mathrm{CH}_{2} \mathrm{Cl}_{2}$.

UV-Vis spectra of the NLO polymers dissolved in $\mathrm{CH}_{2} \mathrm{Cl}_{2}$ were recorded and the $\lambda_{\max }$ Values are reported in Table 6.1. All NLO polycarbonates studied have a strong charge-transfer band $(450 \mathrm{~nm}-650 \mathrm{~nm})$ in the visible region of the spectrum, with $\lambda_{\max }$ lying between $523 \mathrm{~nm}$ and $526 \mathrm{~nm}$. A typical spectrum is shown in Fig. 6.2. In general a small but consistent blue shift $(5 \mathrm{~nm}-8 \mathrm{~nm})$ compared with that of the TCVDPA chromophore is noticeable for all polymers.

To obtain an estimation of the photostability of the NLO polycarbonates, the straightforward and low-cost qualitative method described in Chapter 3 was used. It concerns the monitoring of the decrease in absorbance during irradiation of oxygensaturated solutions of chromophore-containing polymers in $\mathrm{CDCl}_{3}$ with visible white light.

Photobleaching tests were carried out for the chromophore-containing 14-A and 15-A polycarbonates, PMMA-DR1, and polyurethane-DANS (PU-DANS) polymers. From Fig. 6.3 it can be seen that the 14-A and 15-A polycarbonates, containing the highly photostable TCVDPA chromophore, have an exceptional stability under the experimental condition used. In fact, only $5 \%$ decay of the original absorbance was noticed after irradiation for $130 \mathrm{~min}$. There is no significant difference in the photobleaching behavior between the two polycarbonate polymers, indicating the minor influence of the polycarbonate backbone on the photobleaching process. In comparison, the PMMA-DR1 and PU-DANS polymers, containing stilbene-based and azo-based chromophores, show significantly faster degradation rates. From these results it can be concluded that the NLO polycarbonates described in this chapter are among the most photostable structures ever reported in literature. 


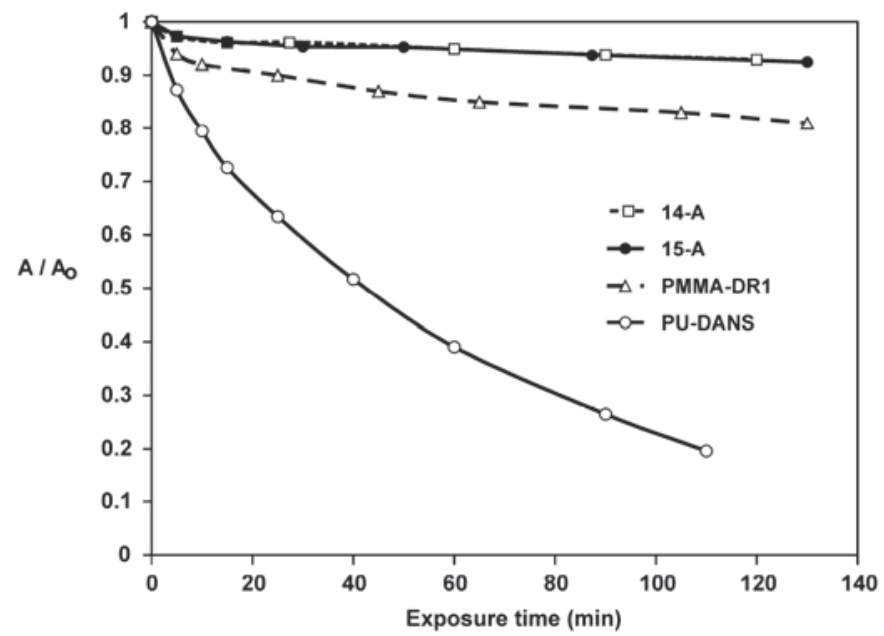

Figure 6.3: Photo-bleaching curves of the 14- $A$ and 15-A polycarbonates, PMMA-DR1, and PUDANS polymers in solution. $A$ is the absorbance at time $t$ and $A_{0}$ is the initial absorbance. The photolyse experiments were carried out in $10 \mathrm{~mm}$ quartz cuvettes with light from a 75 Watt halogen lamp. The samples were irradiated at a distance of $10 \mathrm{~cm}$ from the light source and were shielded from daylight.

All polymers described have good solubility in common organic solvents, such as cyclopentanone. Thin films for EO measurements were prepared as described in Chapter 3 , starting from solutions of the NLO polycarbonates at $10-15 \mathrm{wt} \%$ in cyclopentanone. The $r_{33}$ values, reported in Table 6.1, were measured using a Teng-Man simple reflection technique at a wavelength of $830 \mathrm{~nm}$ (Chapter 3).

As an example, Figure 6.4 shows the $r_{33}$ values measured at different poling temperatures for the 14-A polycarbonate polymer. The poling curves of all polycarbonates show as a general trend the strong dependence of the $\mathrm{EO}$ coefficient on the poling temperature. The measured $\mathrm{r}_{33}$ value increases linearly with the poling temperature, reaching a maximum at $10^{\circ} \mathrm{C}-15^{\circ} \mathrm{C}$ below the $\mathrm{T}_{\mathrm{g}}$ of the material. After this point, no further increase or even a decrease of the EO coefficient is observed. Therefore these temperatures were chosen as the poling temperature of the devices. 


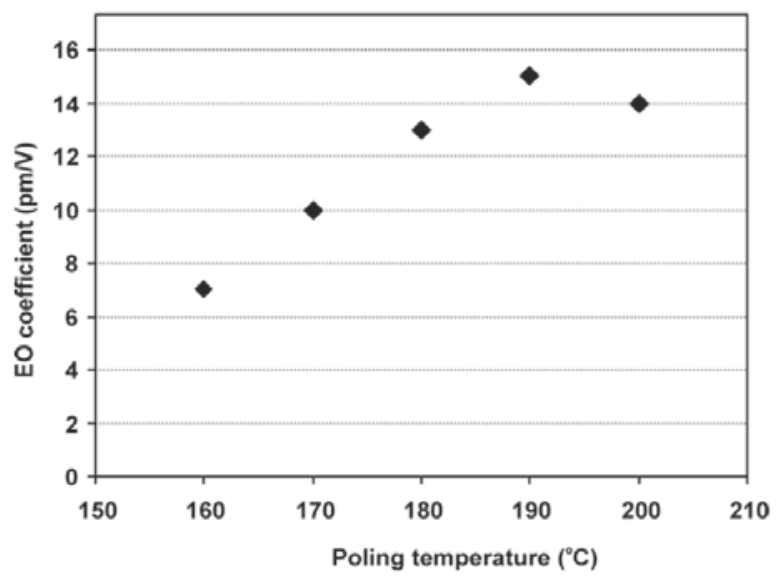

Figure 6.4: Values of the $r_{33}$ coefficient of 14-A measured at different poling temperatures to optimize the poling process. The chromophore concentration in the polymer was $39 \mathrm{wt} \%$ and its $T_{8}$ was $206^{\circ} \mathrm{C}$.

The 14-A and 14-Z polymers, having the highest chromophore loading density of the series (being $39.8 \mathrm{wt} \%$ and $38.6 \mathrm{wt} \%$, respectively), have an $\mathrm{r}_{33}$ coefficient of $15 \mathrm{pm} / \mathrm{V}$. In contrary to our expectation polymer 15 gave a higher value of the measured $\mathrm{r}_{33}$ compared with polymer 16 . The $\mathrm{r}_{33}$ values of the polymer $15-\mathrm{A}(33 \mathrm{pm} / \mathrm{V})$ and $15-\mathrm{Z}(31 \mathrm{pm} / \mathrm{V})$ are comparable to the extrapolated values from the guest host system using the fluorinated TCVDPA chromophore (C5 Chapter 3) in which the effect of the intermolecular interactions can be almost neglected. In the case of the main chain and side chain polymers in which the chromophore is covalently attached to the polymer, the poling efficiency is mainly determined by segmental motion of the backbone and the flexibility of the side chain $[17,18]$. In the case of guest host systems, the poling efficiency is mainly determined by intermolecular interaction. Hence it becomes highly difficult to compare the $r_{33}$ values of these two systems directly. It can, however, be stated that the ease of the chromophore motion under the poling field is not constrained by its attachment to the polymer in polymers 15-A and 15-B.

\subsubsection{Relaxation behavior}

One of the important parameters determining the practical applicability of poled polymers is the stability of the induced polar orientation. This is usually studied through the temporal behavior of the electro-optic coefficient $\left(r_{33}\right)$ or the decay in the generated second harmonic signal at elevated temperatures. As the chromopores in the polymer feel different environments, for example, the free volume in the polymer is not homogeneous, the relaxation cannot be easily defined by a single exponential function. The relaxation of 


\section{NLO Polycarbonates}

the electro-optic activity can be described by the Kohlrausch-Williams-Watts (KWW) [19, 20] stretched exponential function given by

$$
r_{33}(t)=r_{33}(0) \exp \left[-(t / \tau)^{\beta}\right]
$$

where $\tau$ is the characteristic time of relaxation, and $\beta$ is a constant which defines the distribution of the time constant (departure from the monoexponential behavior).

The relaxation of poled polymers is also often described by a bi-exponential function [21, 22] as,

$$
r_{33}(t) / r_{33}(0)=R 1 \exp \left[-\left(t / \tau_{1}\right)\right]+R 2 \exp \left[-\left(t / \tau_{2}\right)\right]
$$

where, $\tau_{1}$ and $\tau_{2}$ are the time constants of different relaxation mechanisms which are operative and R1 and R2 their corresponding rates. The constant $\tau_{1}$ is called the short relaxation time and is related to the orientational relaxation. The other constant $\tau_{2}$ is the long characteristic relaxation time related to the diffusion of the chromophore in the polymer matrix (for guest-host systems) which is more temperature sensitive. All these parameters depend on the measurement temperature.

Some people also use tri exponential functions [23] with characteristic relaxation times $\tau_{1}$, $\tau_{2}$ and $\tau_{3}$ and corresponding rates R1, R2 and R3. This kind of tri-exponential function explains well the behavior of polymers in which different segmental motions are possible. Fig. 6.5 shows the different possible segmental motions in a side chain polymer. The relaxation is determined by the flexibility of the main chain and side chain. The relaxation mode $\beta$ depends on,

(i) the size of the local free volume around the chromophore,

(ii) degree of decoupling between the choromophore and the polymer backbone,

(iii) temperature.

The rapid relaxation which occurs once the poling field is switched off is mainly because of the $\beta$ relaxation mechanism. The subsequent relaxation is dominated by the a mode. The $\gamma$ relaxation mechanism involves ultra slow reorientation motions of the chromophores about their axis. This is normally not observed in amorphous isotropic polymers. 


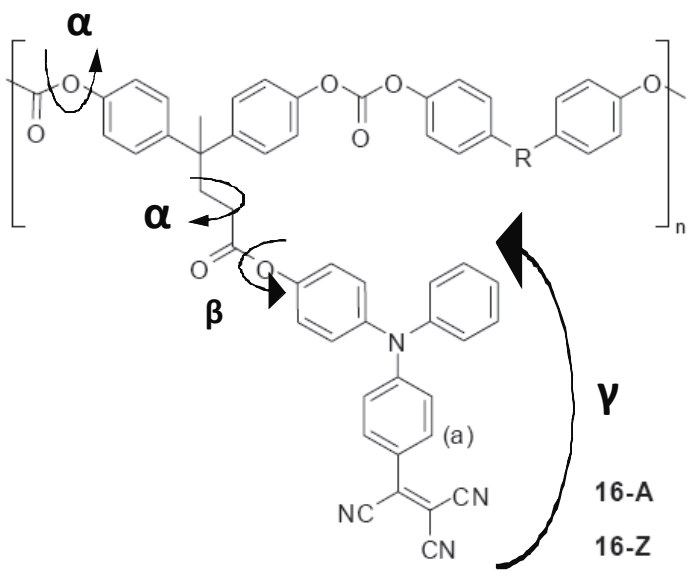

Figure 6.5: Different mechanisms affecting the relaxation behavior of poled polymers with covalently linked chromophores.

To study the effect of the chromophore-to-polymer binding mode and the attachment flexibility on the temporal stability of the dipole alignment, the decay of the EO coefficient at $50^{\circ} \mathrm{C}$ was followed over time for poled samples of the 14-A, 15-A, and 16-A polycarbonates. From Figs. 6.6 to 6.9 it is clear that 14-A and 15-A have the highest temporal stability, retaining over $90 \%$ of their original EO response after $300 \mathrm{~h}$. Differently, for 16-A (figure 6.7) a faster decay was observed, retaining only $74 \%$ of the starting value. These trends might be understood considering the two factors that contribute to the decay:

(i) the $\mathrm{T}_{\mathrm{g}}$ of the polymer, that is related to the energy needed for the whole backbone to increase its mobility and

(ii) the chromophore binding mode, that is related to its degree of freedom and therefore with the energy needed for the single chromophore to reorient.

While the first factor is predominant at high temperatures (less than $50^{\circ} \mathrm{C}$ to the $\mathrm{T}_{\mathrm{g}}$ ), the latter has a big influence at lower temperatures and at room temperature. Since all polycarbonates in this study have a $T_{g}$ higher than $160^{\circ} \mathrm{C}$ it is assumed that at $50^{\circ} \mathrm{C}$ the observed decay is due to differences in the chromophore binding mode.

All the above mentioned mechanisms are accelerated at high temperatures. The time constants exhibit an Arrhenius thermally activated behavior given by,

$$
\tau(T)=A^{*} \exp [E / R T]
$$

where, $E$ is the thermal activation energy and $\mathrm{A}$ is a constant. 


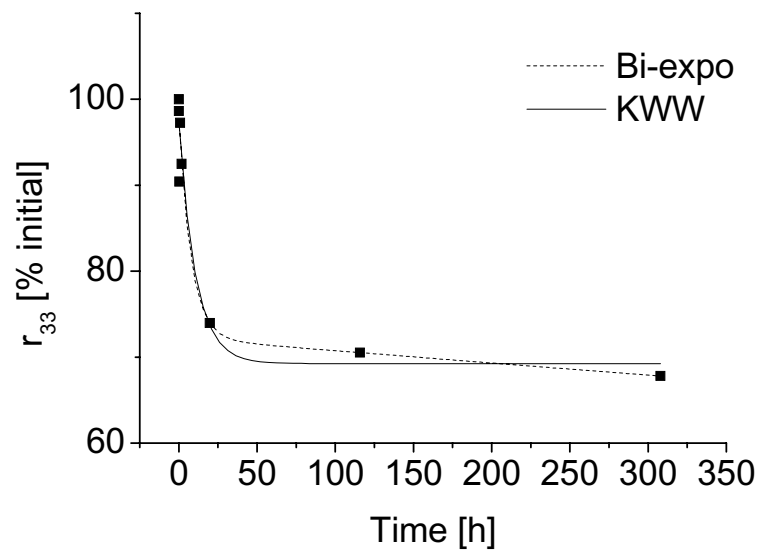

Figure 6.6: Relaxation behavior of PS-17 wt\% TCVDPA guest host system at $50^{\circ} \mathrm{C}$.

Fig. 6.6 shows the relaxation of the poled PS-TCVDPA guest-host system at $50^{\circ} \mathrm{C}$ monitored by measuring $r_{33}$ as a function of time. It can be seen that bi-exponential function gives a better fit compared with KWW. The short and long characteristic relaxations times were $8 \mathrm{~h}$ and $4890 \mathrm{~h}$ respectively.

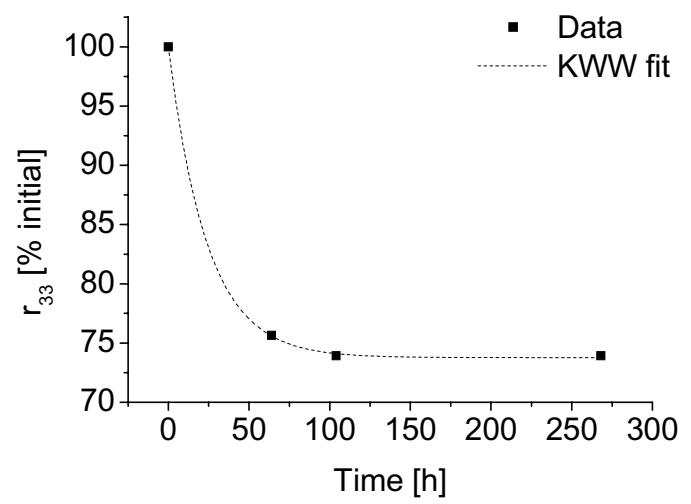

Figure 6.7: Relaxation behavior of $16-\mathrm{A}$ at $50^{\circ} \mathrm{C}$.

From Fig. 6.7 it can be seen that the KWW function gives a good fit in the case of side chain polymers with a distribution of the time constant close to one approaching a single exponential decay. This shows that mainly one relaxation mechanism is dominant at least during the initial stages of the decay arising from the $\beta$ relaxation. 


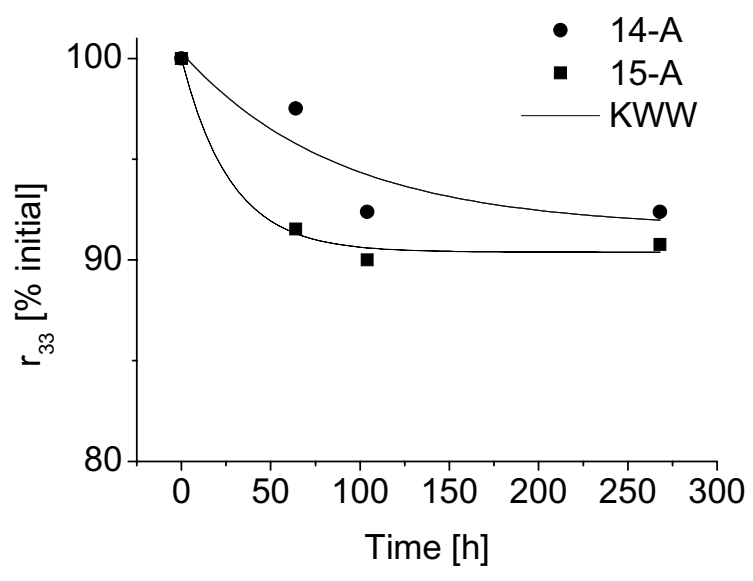

Figure 6.8: Relaxation behavior of $14-A$ and $15-A$ at $50^{\circ} \mathrm{C}$.

In the case of 14-A and 15-A KWW fits better than the bi-exponential function (Fig. 6.8). A more rapid decay in the case of 15-A could be because of difference in $\mathrm{T}_{\mathrm{g}}$ which is about 40 ${ }^{\circ} \mathrm{C}$ less compared with 14-A. Also in these cases (Fig. 6.8) the major contribution is from the $\beta$ relaxation mechanism with a small contribution from the a relaxation which requires high activation energy.

A comprehensive overview of the relaxation behaviors of 14-A, 15-A and 16-A is shown in Fig. 6.9. In case of the 14-A and 15-A polymers the chromophore is part of the polymer main-chain being covalently bound at two positions. Therefore it requires more energy to relax back to the centrosymmetric state. However, in case of 16-A the NLO chromophore is attached as a side appendage to the backbone, giving to it a greater degree of freedom to reorient independently, without requiring the additional energy needed for the polycarbonate structure to move with it.

It can be concluded that flexibility of the backbone determined by the $\mathrm{T}_{\mathrm{g}}$ plays a major role in poling. But stability of the dipole alignment close to the room temperature increases with the number of chromophore-to-polymer attachment points. Moreover, direct incorporation of the TCVDPA chromophore into the polymer backbone gives a higher temporal stability than being attached through a spacer. In the case of covalently linked chromophores only one relaxation mechanism is dominant close to room temperature whereas in the case of guest-host systems clearly two relaxation mechanisms can been seen leading to a rapid decay even at room temperature. 


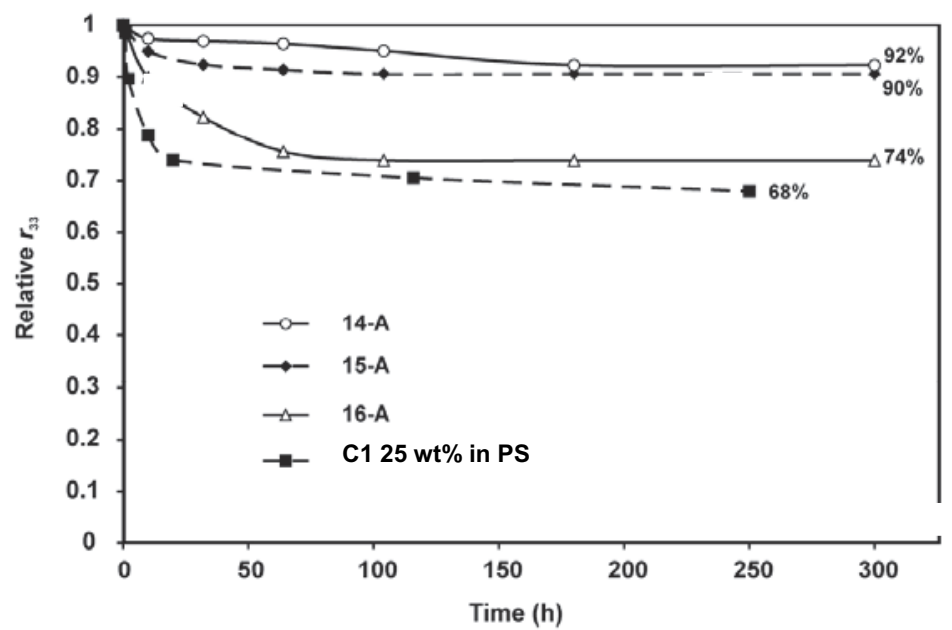

Figure 6.9: Comparison of the relaxation curves at $50^{\circ} \mathrm{C}$ for different $E O$ polymer systems.

\subsection{Functionalization of APC with high $\mu \beta$ chromophores}

PC can also be functionalized with other highly nonlinear chromophores. As most of the high $\mu \beta$ chromophores cannot withstand the harsh polymerizing conditions, a prepolymer was made with reactive sites for attachment of choromophores. The schematic of this approach is shown in Fig. 6.10. PC-Z (Table 6.1) was functionalized with DR1, FTC (see Chapter 2 Table 2.1) and TCV (momohydroxyalkyl TCVDPA type chromophore) [24] chromophores. Fig. 6.11 shows the UV-Vis absorption spectrum of these polymers with their charge transfer absorption peak. To minimize the optical loss contributed from absorption, it is important to have the material with its absorption band edge far away from the operating wavelength of EO devices. Therefore, the PC-Z-FTC polymers are suitable for use at $1300 \mathrm{~nm}$ and $1550 \mathrm{~nm}$, while the polymers containing the DR1 and TCV chromophores can also be used at $830 \mathrm{~nm}$.

Thin films for EO measurements were prepared as described in Chapter 3, starting from solutions of the NLO polycarbonates at 10-15 $\mathrm{wt} \%$ in cyclopentanone. The polymers were soluble in cyclopentanone after one day of stirring on a mechanical stirrer. The solution was the pre-filtered using a filter paper and then using a 0.2 micron filter. The $\mathrm{r}_{33}$ values were measured using Teng-Man technique. An $\mathrm{r}_{33}$ of $22 \mathrm{pm} / \mathrm{V}$ and $38 \mathrm{pm} / \mathrm{V}$ were measured respectively for DR1 and FTC functionalized PC respectively. For the PC-ZTCV polymers it was not possible to obtain optical quality thin films, due to the poor solubility in spin-coating solvents, making EO measurements impossible. 
a<smiles>CCCCCOC(=O)COC(=O)CC(CCC(=O)O)(c1ccc(OC(C)=O)cc1)c1ccc(OC(C)C)cc1</smiles>

b

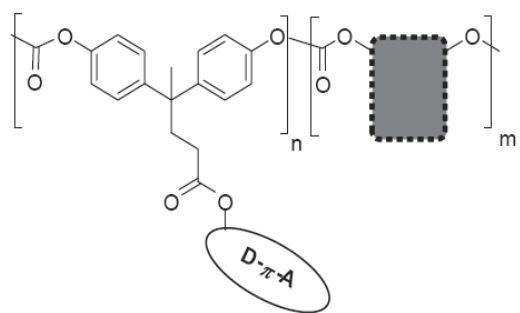

Figure: 6.10: Attachment of NLO chromophores to APC, pre polymerizization (a), post functionalization (b).

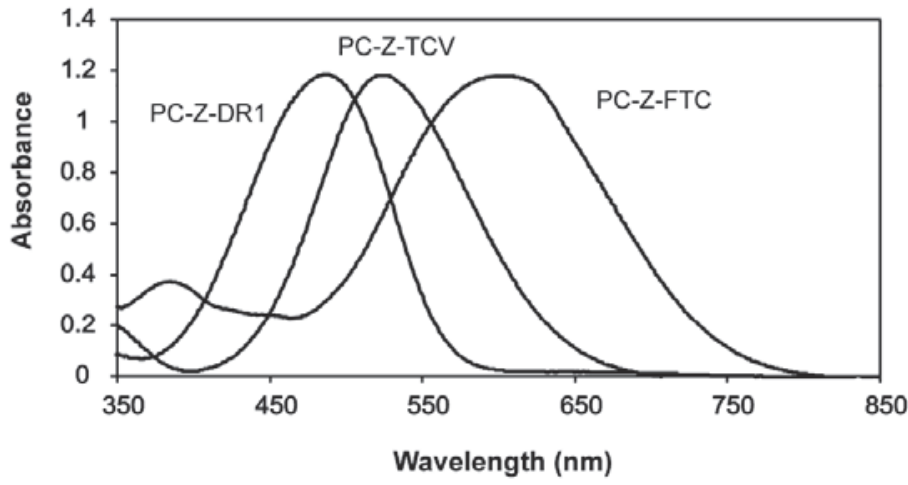

Figure 6.11: UV-Vis spectrum of PC-Z-NLO polymers.

A versatile, generally applicable synthetic methodology for side-chain NLO polycarbonates was developed. This represents the first example of covalent incorporation of NLO chromophores to a pre-polymerized polycarbonate backbone. This methodology allows to adjust the polymer backbone structure and to vary the chromophore loading level in order to fine-tune the physical properties of the resulting material. The resulting NLO polycarbonates possess a high thermal stability, good solubility and can be easily 


\section{NLO Polycarbonates}

processed into films of excellent quality. The combination of these properties provides a great promise for the development of EO devices.

\subsection{Conclusions}

The $\mathrm{C} 1$ chromophore was incorporated in polycarbonate attaching it to the polymer backbone to enhance the $\mathrm{T}_{\mathrm{g}}$ of the material. $\mathrm{C} 1$ was attached to the main chain, as side chain and to the main chain with a spacer. The one with spacer was found to have the highest poling efficiency with an $\mathrm{r}_{33}$ of $33 \mathrm{pm} / \mathrm{V}$ indicating that some amount of flexibility of the backbone is required for the alignment of the chromophores. The relaxation measurements reveal that the chromophore relaxation depends both on the $T_{g}$ and on the chromophore binding mode. At lower temperatures compared to the $T_{g}$ the relaxation process is mainly determined by the chromophore binding mode while at relatively higher temperatures the $T_{g}$ of the material has a dominant effect on the relaxation.

\subsection{References}

[1] L. R. Dalton, A. Harper, A. Ren, F. Wang, G. Todorova, J. Chen, C. Zhang, M. Lee, Ind. Eng. Chem. Res., 38, p8, 1999.

[2] L. R. Dalton, J. Phys.: Condens. Matter, 15, pR897, 2003.

[3] M. E. Wright, S. Fallis, A. J. Guenthner, L. C. Baldwin, Macromolecules, 38, p10014, 2005.

[4] C. Zhang, L. R. Dalton, M. C. Oh, H. Zhang, W. H. Steier, Chem. Mater., 13, p3043, 2001.

[5] T. A. Chen, A. K. Y. Jen, Y. M. Cai, Chem. Mater, 8, p607, 1996.

[6] A. K. Y. Jen, X. M. Wu, H. Ma, Chem. Mater, 10, p471, 1998.

[7] H. Ma, X. J. Wang, X. M. Wu, S. Liu, A. K. Y. Jen, Macromolecules, 31, p4049, 1998.

[8] M. C. Oh, H. Zhang, C. Zhang, H. Erlig, Y. Chang, B. Tsap, D. Chang, A. Szep, W. H. Steier, H. R. Fetterman, L. R. Dalton, IEEE J. Sel. Top. Quantum Electron., 7, p826, 2001.

[9] Y. J. Cheng, J. Luo, S. Hau, D. H. Bale, T. D. Kim, Z. Shi, D. B. Lao, N. M. Tucker, Y. Tian, L. R. Dalton, P. J. Reid, A. K. Y., Jen Chem. Mater., 19, p1154, 2007.

[10] C. C. Chang, C. P. Chen, C. C. Chou, W. J. Kuo, R. J. Jeng, J. Macromol. Sci., Polym. Rev., 45, p125, 2005.

[11] C. Samyn, T. Verbiest, A. Persoons, Macromol. Rapid Commun., 21, p1, 2000. 
[12] R. H. Woudenberg, T. O. Boonstra, J. W. Ladage, U. E. Wiersum, Non-linear optical polycarbonates, 96, p28493, 1999.

[13] A. Galvan-Gonzalez, M. Canva, G. I. Stegeman, R. Twieg, K. P. Chan, T. C. Kowalczyk, X. Q. Zhang, H. S. Lackritz, S. Marder, S. Thayumanavan, Opt. Lett., 25, $p 332,2000$.

[14] A. Galvan-Gonzalez, M. Canva, G. I. Stegeman, L. Sukhomlinova, R. J. Twieg, K. P. Chan, T. C. Kowalczyk, H. S. Lackritz, J. Opt. Soc. Am. B 17, p1992, 2000.

[15] M. H. Davey, V. Y. Lee, L. M. Wu, C. R. Moylan, W. Volksen, A. Knoesen, R. D. Miller, T. J. Marks, Chem. Mater, 12, p1679, 2000.

[16] X. Li, A. F. Yee, Macromolecules, 36, p9421, 2003.

[17] D. Briers, L. De Cremer, G. Koeckelberghs, S. Foerier, T. Verbiest, C. Samyn, Macromol. Rapid Commun., 28, p942, 2007.

[18] M. D. Ediger, Annu. Rev. Phys. Chem., 51, p99, 2000.

[19] F. Kohlrausch, Pog. Ann. Physk., 91, p179, 1847.

[20] G. Williams, D. C. Watts, Trans. Faraday Soc., 66, p80, 1970.

[21] H. Hampsch, J. Yang, G. Wong, J. Torklson, Macromolecules, 23, p3640, 1990.

[22] G. Linsay, R. Henry, J. Hoover, A. Knoesen, M. Mortazavi, Macromolecules, 25, p4888, 1992.

[23] C. Noel, F. Kajzar, P. N. Prasad, J. E. Mark, S. H. Kandil, Z. Kafafi, Highly orientable liquid crystalline polymers for quadratic non-linear optics, Proceedings of the fourth international conference on frontiers of polymers and advanced materials, Plenum Press, New York, p275, 1999.

[24] M. Faccini, , Supramolecular Chemistry and technology, chapter 5, PhD thesis, University of Twente, Enschede, The Netherlands. 
NLO Polycarbonates 


\section{Summary and conclusions}

The research described in this thesis deals with the development of new thermally and photochemically stable EO materials. TCVDPA was made as the chromophore of choice with a simple benzene ring as a л-electron bridge between a tricynovinyl acceptor and an amino donor.

The TCVDPA chromophore was modified with different bulky side groups (C1-C6). The effect of these side groups in reducing the intermolecular interactions during poling is studied by incorporating these chromophores in Polysulfone as guest-host system. Different bulky groups were found to exhibit varying degree of influence in reducing the intermolecular interactions. Modifying the chromophore with bulky groups did not seem to have effect on the hyperpolarizability of the molecule. The bare chromophore $\mathrm{C} 1$ shows a linear increase in $\mathrm{r}_{33}$ upto about $15 \mathrm{w} \%$ after which the intermolecular interaction starts which causes a flattening of the $\mathrm{r}_{33}$ curve. The C3 chromophore with tert-butly groups shows a linear increase in $\mathrm{r}_{33}$ upto $35 \mathrm{w}_{\text {eff }} \%$. A maximum $\mathrm{r}_{33}$ of $24 \mathrm{pm} / \mathrm{V}$ was measured for this chromophore at $35 \mathrm{w}_{\text {eff }} \%$ which is about twice as high as the measured value for $\mathrm{C} 1$. The $\mathrm{C} 5$ chromophore which is more bulkier than $\mathrm{C} 3$ also showed a similar effect as $\mathrm{C} 3$ but with an increased slope of the $\mathrm{r}_{33}$ curve. As there cannot be any intermolecular interactions present in the linear regime of the $\mathrm{r}_{33}$ curve this difference in the slope could be because of the site isolation effect. The maximum value of $r_{33}$ which was measured with the C5 chromophore was $24 \mathrm{pm} / \mathrm{V}$ at $30 \mathrm{w}_{\text {eff }} \%$. The chromophore C6 which was the bulkiest in the whole series gave a maximum $\mathrm{r}_{33}$ of only $5 \mathrm{pm} / \mathrm{V}$ at $10 \mathrm{w}_{\text {eff }} \%$. It was not possible to incorporate higher concentration of this chromophore because of solubility problems and segregation effects. The $\mathrm{C} 5$ chromophore was found to have the optimum bulkiness for high poling efficiency and also at the same time enabling incorporation of high concentration. All the chromophores were found to have a plasticizing effect on the host polymer reducing its $\mathrm{T}_{\mathrm{g}}$. The smaller the chromophore size the higher is this effect. The $\mathrm{C} 5$ chromophore is also advantageous in this respect that the plasticizing effect is less compared to C3. Photostability measurements show TCVDPA and its modifications are more stable than other known chromophores like DR1. For $20 \mathrm{w} \% \mathrm{C} 1$ in polysulfones slab waveguiding losses were measured to be $1.7 \mathrm{~dB} / \mathrm{cm}$ at $1523 \mathrm{~nm}$ and $6 \mathrm{~dB} / \mathrm{cm}$ at $850 \mathrm{~nm}$. Both the $\mathrm{T}_{\mathrm{g}}$ and the chromophore size is found to have an effect on the relaxation behavior after the poling field is removed.

A design was made for laterally coupled microrings with PMMA-DR1 as the core material and VSC as the cladding layers. The minimum possible bending radius before the radiation losses increases above $1 \mathrm{~dB} / \mathrm{cm}$ was found to be $200 \mu \mathrm{m}$. Simulations showed that a sub micron gap between the ring and the straight waveguide is required to achieve the required coupling. With a racetrack design it is possible to increase the coupling between the ring and the bus waveguide at a gap of about $1 \mu \mathrm{m}$. Photodefinition of inverted ridges in VSC showed that it was not possible to make inverted ridges less than 3 $\mu \mathrm{m}$ wide and it was not possible to get the $\mu \mathrm{m}$-wide gap open. By embossing it was possible to open the $1 \mu \mathrm{m}$ gap, but removal of the master posed serious problems as it distorted the embossed structures. By using RIE inverted ridges of the ring design were able to be made with gap size not less than $2 \mu \mathrm{m}$ and hence no ring response could be measured because of insufficient coupling. The $1 \mu \mathrm{m}$ gap was made possible with the hybrid approach by defining inverted ridges in $\mathrm{SiO}_{2}$ by $\mathrm{RIE}$ and filling them with PMMA- 


\section{Summary and conclusions}

DR1 by spin coating. The racetrack made by this approach showed a through port dip of $12 \mathrm{~dB}$ and a finesse of 6 . The ring losses were about $25 \mathrm{~dB} / \mathrm{cm}$.

Photodefinition of microring resonators were done with SU8 as the host material, exploiting the low UV absorption window of the TCVDPA chromophore. Poling of such a system is generally very difficult because of the presence of highly conducting species in the photoinitiator. Poling (of the chromophore) and crosslinking (of the host) were optimized to achieve maximum $r_{33}$ value. The effect of neutralization of the photoacid by TCVDPA was compensated by an excess of photoacid to the commercially available composition of SU8. Poling experiments failed when the film is either completely uncrosslinked or was very poor in a completely crosslinked film. Best $\mathrm{r}_{33}$ values were measured by carrying out poling and crosslinking simultaneously by increasing the temperature in a controlled manner. An $\mathrm{r}_{33}$ of about $6 \mathrm{pm} / \mathrm{V}$ was measured with $15 \mathrm{w} \%$ C1 in SU8 which is comparable with values obtained in Polysulfone guest-host system. Microring resonators were made by initially crosslinking an SU8-15 w\% TCVDPA film at $60{ }^{\circ} \mathrm{C}$ to define the structures. A gap of $500 \mathrm{~nm}$ was possible. Channel waveguiding losses were measured to be $5 \mathrm{~dB} / \mathrm{cm}$. This is an significant improvement compared with 16 $\mathrm{dB} / \mathrm{cm}$ channel losses in a waveguide made by RIE. The microrings showed a throughport dip of $11 \mathrm{~dB}$ and the ring losses were $25 \mathrm{~dB} / \mathrm{cm}$. The EO modulation in these rings was demonstrated at $10 \mathrm{MHz}$ and an in in-device $\mathrm{r}_{33}$ of $11 \mathrm{pm} / \mathrm{V}$.

The $\mathrm{C} 1$ chromophore was incorporated in polycarbonate attaching it to the polymer backbone to enhance the $\mathrm{T}_{\mathrm{g}}$ of the material. $\mathrm{C} 1$ was attached to the main chain, as side chain and to the main chain with a spacer. The one with spacer was found to have the highest poling efficiency with an $\mathrm{r}_{33}$ of $33 \mathrm{pm} / \mathrm{V}$ indicating that some amount of flexibility of the backbone is required for the alignment of the chromophores. The relaxation measurements reveal that the chromophore relaxation depends both on the $T_{g}$ and on the chromophore binding mode. At lower temperatures compared to the $\mathrm{T}_{\mathrm{g}}$ the relaxation process is mainly determined by the chromophore binding mode while at relatively higher temperatures the $\mathrm{T}_{\mathrm{g}}$ of the material has a dominant effect on the relaxation.

In conclusion it can be said that a new chromophore had been investigated for its applicability as an EO material. The EO polymers presented in this thesis are among the most photostable structures ever reported representing a step forward towards stable low cost EO devices. 


\section{Appendix 1: Process flow for Hybrid approach}

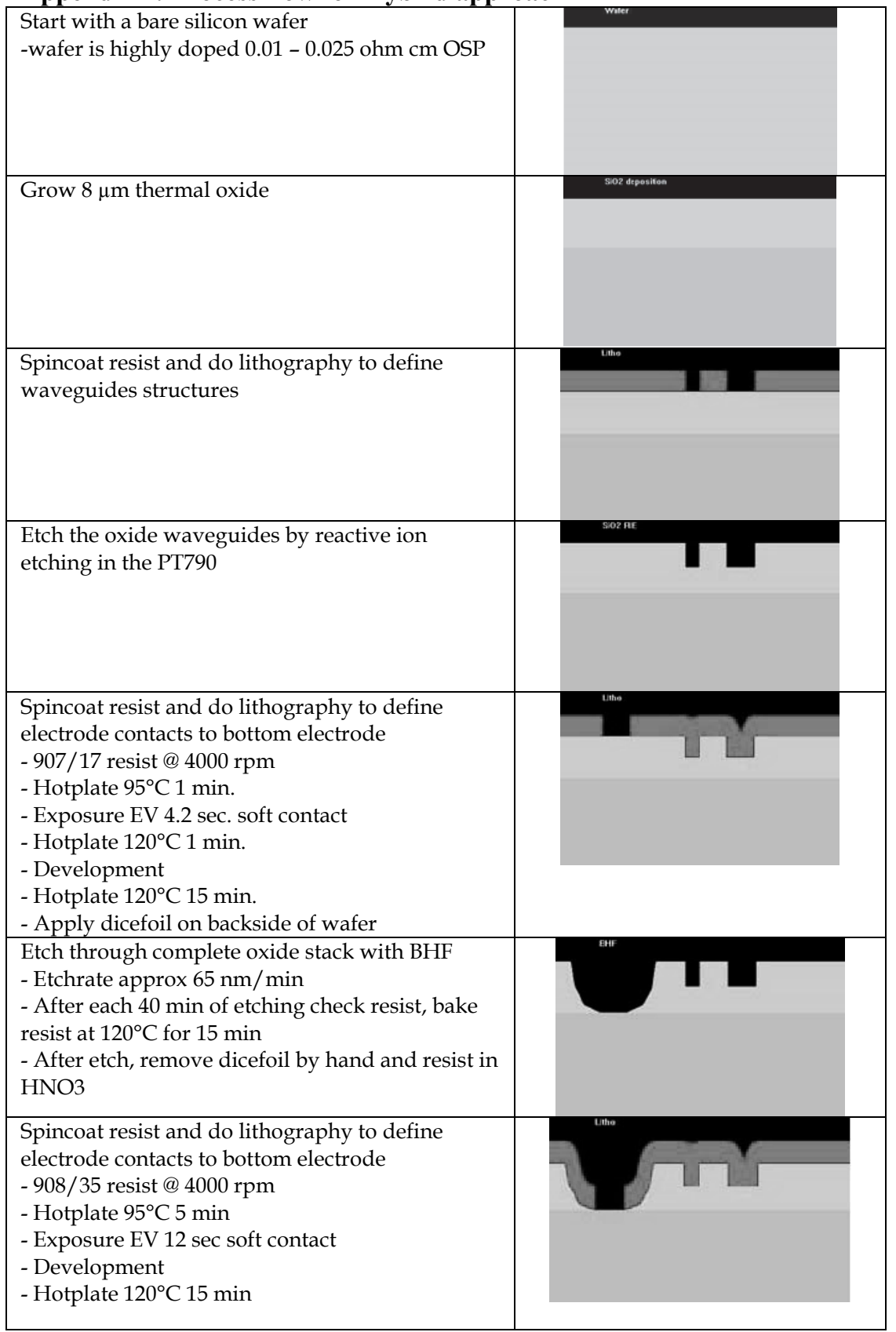




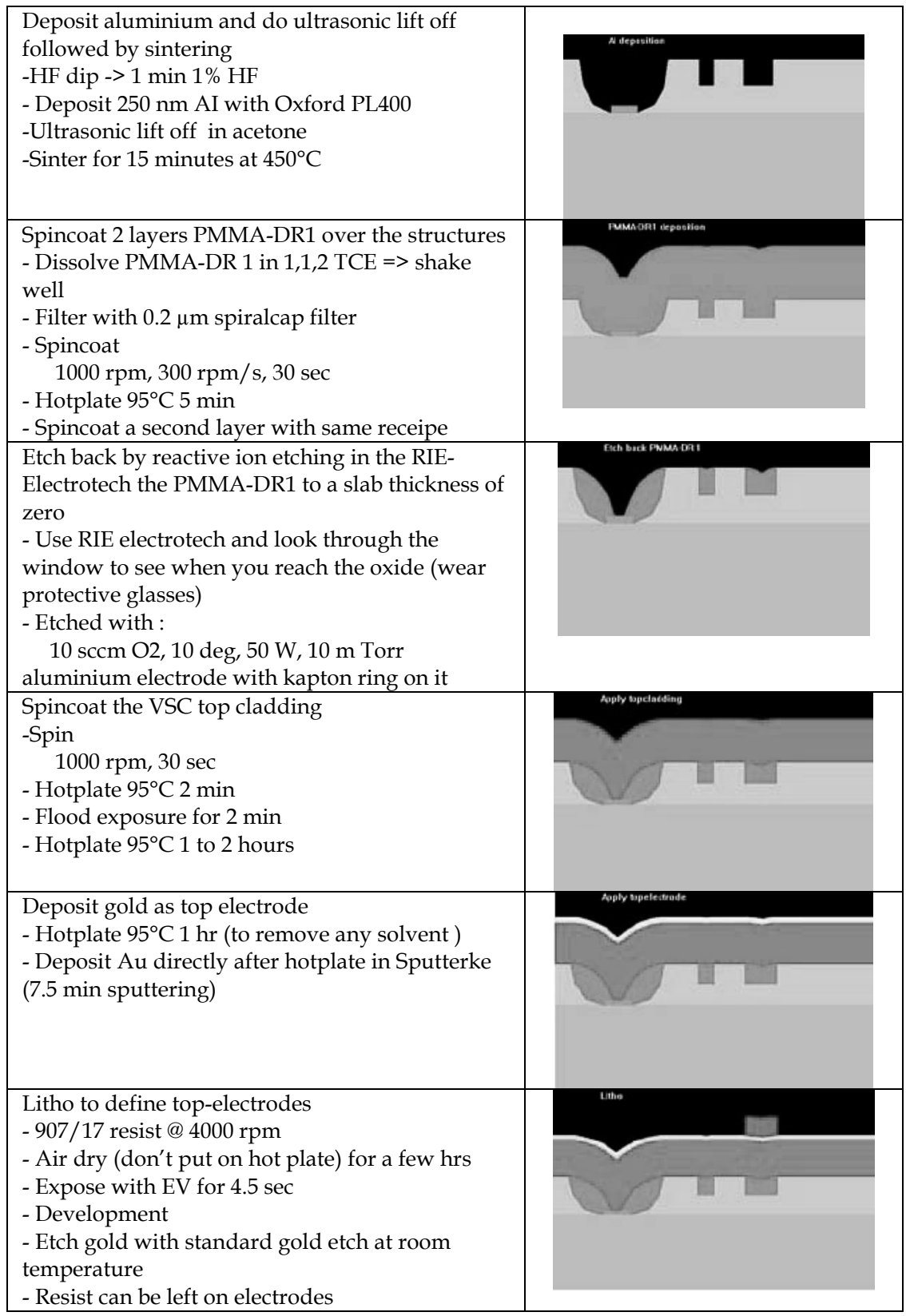




\title{
Microring resonator based modulator made by direct photodefinition of an electro-optic polymer
}

\author{
M. Balakrishnan, ${ }^{1, a}$ M. Faccini, ${ }^{2}$ M. B. J. Diemeer, ${ }^{1, b}$ E. J. Klein,1 G. \\ Sengo, ${ }^{1}$ A. Driessen, ${ }^{1}$ W. Verboom, ${ }^{2}$ and D. N. Reinhoudt ${ }^{2}$ \\ ${ }^{1}$ Integrated Optical Microsystems, MESA+ Institute for Nanotechnology, P.O. Box 217, 7500 AE \\ Enschede, The Netherlands. \\ ${ }^{2}$ Laboratory of Supramolecular Chemistry and Technology, MESA+ Institute for Nanotechnology, \\ P.O. Box 217, 7500 AE Enschede, The Netherlands. \\ Received 25 January 2008; accepted 25 March 2008; published online 18 April 2008 \\ a Electronic mail: bb_murali@yahoo.com. \\ b Present address: Optisense BV, Enschede, The Netherlands.
}

A laterally coupled microring resonator was fabricated by direct photodefinition of negative photoresist SU8, containing tricyanovinylidenediphenylaminobenzene chromophore, by exploiting the low ultraviolet absorption window of this chromophore. The ring resonator was first photodefined by slight cross-linking. Thereafter, poling to align the chromophores and further cross-linking to increase the glass transition temperature were simultaneously carried out. The material showed excellent photostability and the electro-optic modulation with an $r 33$ of $11 \mathrm{pm} / \mathrm{V}$ was demonstrated at $10 \mathrm{MHz}$. @ 2008 American Institute of Physics. DOI: 10.1063/1.2908914

Already for more than 20 years considerable effort has been put in the development and application of organic nonlinear optical materials for electro-optically (EO) active devices ${ }^{1}$. The interest stems from the potential of these materials for low-cost, high performance integrated optics devices which currently are made mostly by using an expensive inorganic crystalline technology like $\mathrm{LiNbO}_{3}$. The advantage of nonlinear optical polymers for electro-optic modulators are potentially low-cost processing capability, large nonlinear EO effects, low dc and microwave dielectric constant and ultra-fast frequency response ${ }^{2}$. Despite these advantages, commercialization of polymer electro-optic modulators ${ }^{3}$ is still in an early stage as most of the highly nonlinear chromophores are photochemically instable. In this work we demonstrate the realization of a photodefined EO polymer modulator based on a laterally coupled microring resonator (MR) with high photochemical stability. Lateral coupling has the advantage that the fabrication requires only a single masking step, but then with critical features. Especially we observed that the sub-micron gap between the ring and the waveguide cannot be realized by standard reactive ion etching (RIE), whereas direct photodefinition shows much better results. Therefore a special chromophore, tricyanovinylidenediphenylaminobenzene (TCVDPA) with a low ultra-violet (UV) absorption window was used that allowed electric field poling and simultaneous crosslinking of a polymer host.

Polymer EO modulators typically consist of waveguides with an EO polymeric core material and low-loss cladding layers, designed such that the field induced index change in the core is transformed to an intensity change by an interferometric arrangement, in our case a $\mathrm{MR}^{4}$. The core material consists of electro-optic chromophores and the polymer host. The specific chromophore determines the photochemical stability of the device. The 
host material plays a critical role in the geometric lay-out and processing scheme of the device and, by its glass transition temperature, $\mathrm{T}_{\mathrm{g}}$, influences strongly the thermal stability. Polymer EO modulators are mostly structured by RIE ${ }^{5}$, which, however, shows some serious drawbacks. It is difficult to make high resolution structures required for laterally coupled MRs in polymers by RIE and many polymers are easily attacked by the photoresist needed to define the etch pattern. In addition, RIE gives rise to high sidewall roughness leading to undesired light scattering. By photodefinition ${ }^{6}$ of the waveguide core, RIE can completely be avoided. As photodefinition is carried out by irradiation with UV light, the host as well as the chromophore has to be stable and transparent to UV. In the following the TCVDPA chromophore is employed which is reported to have a very high photochemical stability ${ }^{7}$; as host SU8 was used ${ }^{8}$. The low UV absorption window of the TCVDPA chromophore allows the host material to be photodefined 9 .

SU8 processing consists of an UV exposure step to generate the photoacid followed by heating to initiate the crosslinking process which increases the $T_{g}$ of the material. Careful thermal curing allows to control the crosslinking process and to do simultaneous poling. For optimization of the combined crosslinking and poling process, a number of test runs were performed. For this, $2 \mu \mathrm{m}$ thick films were made on transparent (at $830 \mathrm{~nm}$ ) indium tin oxide (ITO) coated glass wafer by mixing $15 \mathrm{wt} \%$ TCVDPA in commercially available SU8 dissolved in $\gamma$-butylactone. In the final devices poling is performed with cladding layers, which in the case of test runs were not used for the sake of simplicity. Different poling procedures were carried out and the EO coefficient $r_{33}$ in the poling direction was measured at $830 \mathrm{~nm}$ using the Teng-Man technique ${ }^{10-11}$ which is a simple reflection technique based on ellipsometry to measure the EO coefficient. Poling was monitored by recording the poling current and temperature as function of time. No $\mathrm{r}_{33}$ value could be measured by poling an uncrosslinked film as the $\mathrm{T}_{\mathrm{g}}$ of SU8 in the uncrosslinked state is so low $\left(\sim 50^{\circ} \mathrm{C}\right)$ that the chromophore relaxation is nearly instantaneous after poling. In the case of films highly exposed to UV for 5 mins at $12 \mathrm{~mW} / \mathrm{cm}^{2}$, rapid crosslinking takes place allowing no time for poling. Fig. 1 exhibits the poling behavior of the SU8-15 wt\% TCVDPA system when it had been UV exposed for $1 \mathrm{~min}$ at $12 \mathrm{~mW} / \mathrm{cm}^{2}$. The slow alignment of the chromophores is reflected by the exponential decay of the poling current during the first 500 seconds. With this relatively small UV dose, the crosslinking process is still slow enough to obtain the poling order. The $T_{g}$ after crosslinking in all the above mentioned cases did not increase above $70^{\circ} \mathrm{C}$ because of neutralization of the photoacid by the chromophores and resulting in incomplete crosslinking. The best poling result was obtained by modifying standard SU8 using an additional $3 \mathrm{wt} \%$ photoacid generator and carrying out poling and crosslinking simultaneously by increasing the poling temperature in a controlled manner to keep pace with the increasing $\mathrm{T}_{\mathrm{g}}$. Up to now an $\mathrm{r}_{33}$ of $5 \mathrm{pm} / \mathrm{V}$ could be obtained in this case with increase in $\mathrm{T}_{\mathrm{g}}$ to $190^{\circ} \mathrm{C}$. 


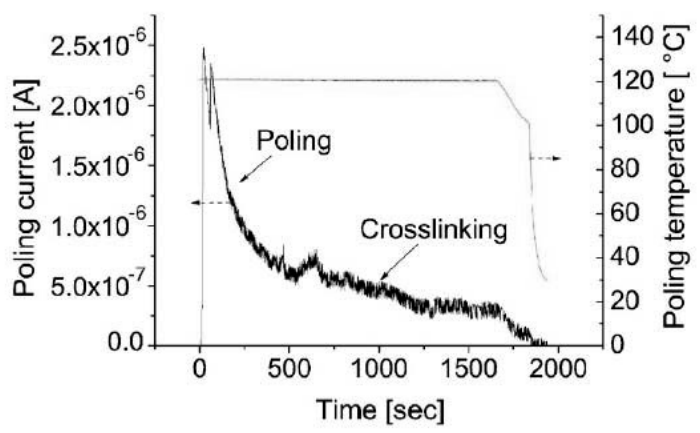

Figure 1: Poling behavior of SU8-15 wt\% TCVDPA film which was slightly exposed to UV prior to poling.

With this optimized procedure EO devices have been realized that consist of a laterally coupled MRs. For the core SU8-15 wt\% TCVDPA ( $\mathrm{n}=1.592$ at $1550 \mathrm{~nm}$ ) was used and VSC (a UV curable epoxy ${ }^{12}$ ) with a refractive index of 1.51 at $1550 \mathrm{~nm}$ as cladding. The radius of the ring was $200 \mu \mathrm{m}$ to keep the bending losses below $1 \mathrm{~dB} / \mathrm{cm}$. The waveguides were $2 \mu \mathrm{m}$ wide and $1 \mu \mathrm{m}$ thick to maintain the monomodal condition. A racetrack kind of structure (Fig. 2) was used for the ring to increase the coupling between the ring and the bus waveguide.

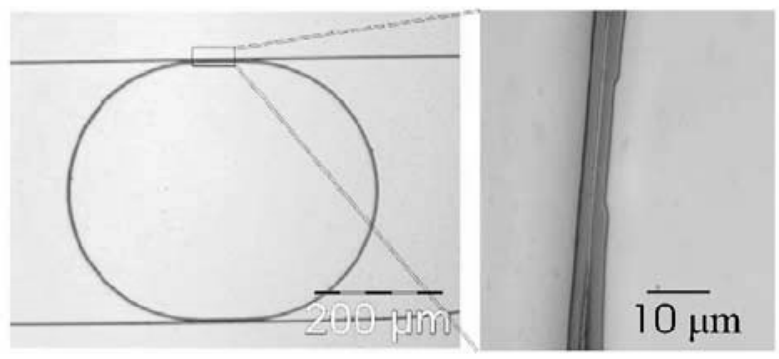

Figure 2: Optical microscope picture of a SU8-15 wt\% TCVDPA MR made by direct photodefinition. The inset shows a closer view of the coupling section.

The fabrication starts with sputter deposition of the bottom electrode, a $250 \mathrm{~nm}$ thick gold layer, on a silicon wafer. A $6 \mu \mathrm{m}$ thick layer of VSC was spun on top of the electrode, exposed to UV and cured at $95^{\circ} \mathrm{C}$. Thereafter the core layer was spun on VSC to form a 1 $\mu \mathrm{m}$ thick film. The doped SU8 layer was exposed to UV through a contact chromium mask with the MR design. After slightly crosslinking the core layer at $60^{\circ} \mathrm{C}$ for 5 minutes, the unexposed regions surrounding the waveguides could be removed in SU8 developer. 
Fig. 3 shows the critical coupling section of the MR with the excellent result of the photodefinition process. A $6 \mu \mathrm{m}$ layer of VSC was spin coated as top cladding. The processing temperature after spinning the top cladding was limited to $60^{\circ} \mathrm{C}$ in order to avoid any additional crosslinking of the core layer. A $250 \mathrm{~nm}$ layer of gold was sputtered and patterned for the purpose of poling and electro-optic modulation in a lumped electrode configuration. Simultaneous poling and crosslinking of the MR was carried out in the same carefully controlled manner as for the unstructured film.

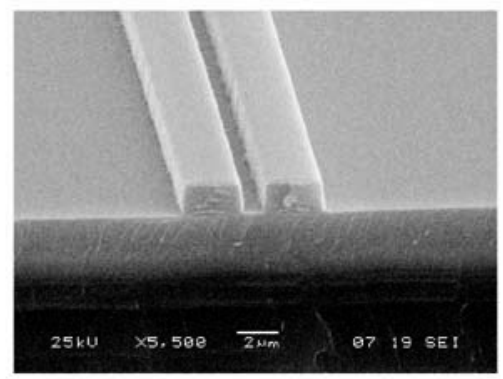

Figure 3: SEM picture showing the coupling section of the MR made by direct photodefinition of SU8-15 wt\% TCVDPA.

Fig. 4 shows the through port response of the MR with a dip of $11 \mathrm{~dB}$, a free spectral range (FSR) of $1 \mathrm{~nm}$ and a finesse of 6 . A ring waveguide loss of $25 \mathrm{~dB} / \mathrm{cm}$ mainly due to the transition losses at the junction between the curved and the straight section of the ring which was not optimized and a field coupling constant of 0.8 were extracted by making a fit to the through port response using Rfit ${ }^{13}$ software. The slab waveguiding loss (mostly absorption) of the core material was measured to be $1.6 \mathrm{~dB} / \mathrm{cm}$ at $1523 \mathrm{~nm}$. In order to asses the losses in the channel waveguide, quantitative image processing was made on pictures obtained with an IR camera with Curveloss software ${ }^{14}$. For comparison waveguides made by RIE of PMMA-DR1 were inspected.

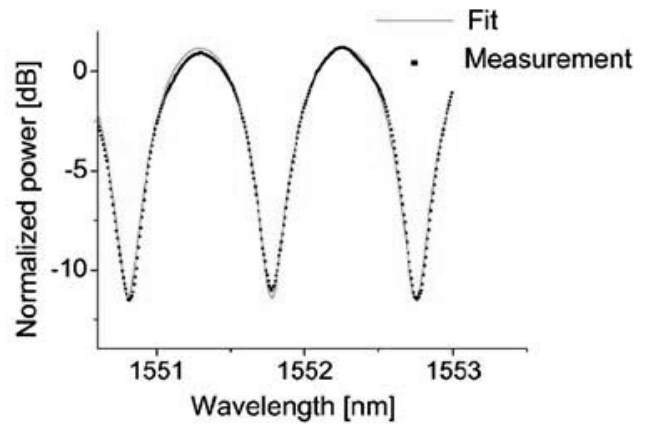

Figure 4: Through port response of the MR shown in Fig. 3 for TM polarization; dotted line: experiment; solid line: fit. 
Fig 5 shows the scatter intensity close to the in-coupling facet when equal power is coupled to both waveguides. It appears that scattering from the photodefined waveguide is about four times less than the from the RIE waveguide. Channel waveguiding losses were measured by analyzing the scattering intensity as a function of the waveguide length and was found to be $5 \mathrm{~dB} / \mathrm{cm}$ for the photodefined waveguide and $16 \mathrm{~dB} / \mathrm{cm}$ for the RIE waveguide.

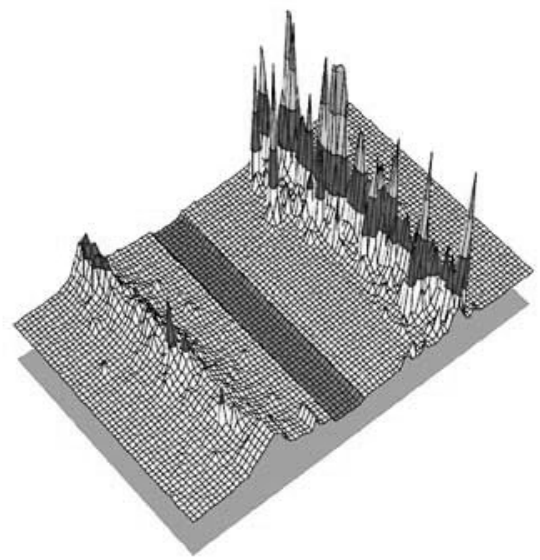

Figure 5: Intensity plot of the scattered light from waveguides with same input power: photodefined waveguide (left) and RIE waveguide (right).

Fig. 6 shows the semi-static electro-optic response observed by applying a saw tooth voltage at a frequency of $1 \mathrm{KHz}$. Increasing the frequency to $10 \mathrm{MHz}, \mathrm{EO}$ modulation could be clearly observed (Fig. 7). In both the cases, $\mathrm{r}_{33}$ was determined to be $\sim 11 \mathrm{pm} / \mathrm{V}$. Because of limitations of the experimental set-up the modulation speed could not be increased beyond $10 \mathrm{MHz}$. This speed, however, is sufficiently high to confirm the pure EO origin of the modulation.

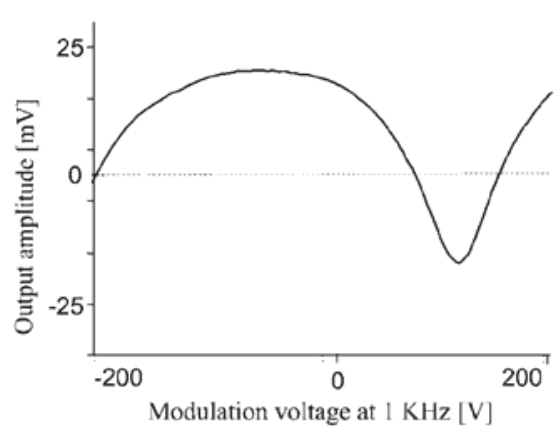

Figure 6: Output at the through port, measured with a photo-detector connected to an oscilloscope, as a function of the applied voltage across the electrodes, showing a shift of a complete FSR. 
The device has an extremely high photostability even without any packaging or shielding to oxygen. As a test light form a tunable laser source at $1550 \mathrm{~nm}$ was coupled to the device. With an insertion loss of $20 \mathrm{~dB}$ and a laser power of $15 \mathrm{~mW}$, power coupled into the waveguide is estimated to be $1.5 \mathrm{~mW}$. After about 100 hours no reduction in $\mathrm{r}_{33}$ was observed. After several weeks the device delaminated from the substrate because of poor adhesion between the gold bottom electrode and the VSC bottom cladding. The problem stems from the photoinitiator for the epoxy ${ }^{15}$. This is a sulfur containing salt and may react with gold forming a so-called weak boundary layer. By covering of the gold by a nonreactive metal, or by applying a sulfur containing adhesion promoter this problem can be solved.

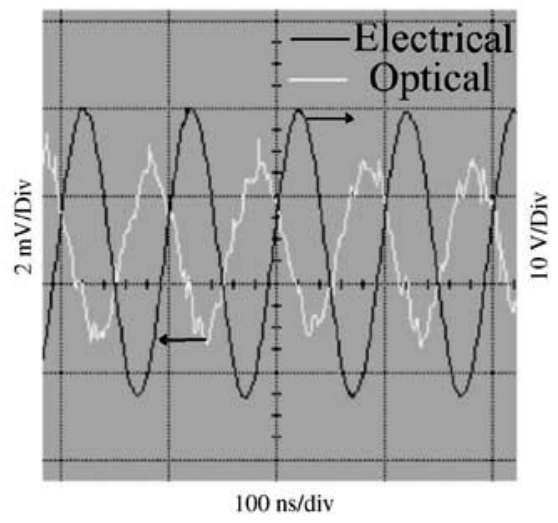

Figure 7: Electro-optic modulation seen at $10 \mathrm{MHz}$ by tuning the wavelength to the point of maximum slope of the through port response. High driving voltage had to be used because of the not yet optimized cladding layers.

In conclusion, a new materials system has been developed by which EO MRs can be directly photodefined using a single masking step. The system shows a high photochemical stability and has a high $T_{g}$. The $r_{33}$ of this material system is promising and can be further increased by using TCVDPA modified with bulky groups which will inhibit intermolecular interactions during poling ${ }^{16}$. This has already been demonstrated ${ }^{9}$ and an $\mathrm{r}_{33}$ as high as $25 \mathrm{pm} / \mathrm{V}$ has been achieved before segregation of the chromophores can start. Also further optimization of crosslinking and poling procedure will lead to higher r33 values.

This research work is supported by the Dutch Technology Foundation STW, Applied Science Division of NWO and the Technology Program of the Ministry of Economic Affairs. We thank Nur Ismail and Marcel Hoekman for their help in the determination of the waveguide losses. 


\section{References}

[1] L. R. Dalton, J. Phys. Condens. Matter, 15, p897, 2003.

[2] M. Lee, H. E. Katz, C. Erben, D. M. Gill, P. Gopalan, J. D. Heber, D. J. McGee, Science, 298, p1401, 2002.

[3] www.lumera.com

[4] A. Leinse, M. B. J. Diemeer, A. Rousseau, A. Driessen, IEEE Photonics Tech. Lett., 17, p2074, 2005.

[5] D. Jin, Proc. SPIE, 4991, p610, 2003.

[6] M. B. J. Diemeer, L. Hilderink, R. Dekker, A. Driessen, IEEE Photonics Tech. Lett., 18, $p 1624,2006$.

[7] A. G. Gonzalez, G. I. Stegeman, A. K. Y. Jen, X. Wu, M. Canva, A. C. Kowalczyk, X. Q. Zhang, H. Z. Lackritz, J. Opt. Soc. Am., B 18, p12, 2001.

[8] M. Nordström, D. A. Zauner, A. Boisen, J. Hübner, Proc. SPIE, 6112, p611206, 2006.

[9] M. Balakrishnan, M. Faccini, M. B. J. Diemeer, W. Verboom, A. Driessen, D. N. Reinhoudt, A. Leinse, Electronics Lett., 42, p51, 2006.

[10] C. C. Teng, H. T. Man, Appl. Phys. Lett., 56, p1734, 1990.

[11] F. Michelotti, G. Nicolao, F. Tesi, M. Bertolotti, Chem. Phys. 245, p311, 1999.

[12] From Surface Specialties Cytec Industries (formerlyUCB).

[13] E. J. Klein, Non-commercial MR fit software, Integrated Optical Microsystems, University of Twente, The Netherlands.

[14] M. Hoekman, Non-commercial Image processing software, Integrated Optical Microsystems, University of Twente, The Netherlands.

[15] A. Hartwig, J. Adhesion Sci. Tech., 17, p1561, 2003.

[16] B. H. Robinson, L. R. Dalton, J. Phys. Chem. A ,104, p4785, 2000. 
APL 2008 paper 


\title{
Photodefinable electro-optic polymer for high speed modulators
}

\author{
M. Balakrishnan, M. Faccini, M. B. J. Diemeer, W. Verboom, A. Driessen, \\ D. N. Reinhoudt and A. Leinse
}

Abstract: Direct waveguide definition of a negative photoresist (SU8) containing tricyanovinylidenediphenylaminobenzene (TCVDPA) as electro-optic (EO) chromophore, has been demonstrated for the first time. This was possible by utilizing the chromophore low absorption window in the UV region allowing crosslinking of the host polymer by exposing to UV light followed by thermal curing. TCVDPA was modified by attachment of bulky side groups. This reduced the intermolecular interactions and resulted in an increased $\mathrm{EO}\left(\mathrm{r}_{33}\right)$ coefficient.

Introduction: Over the past decade the demand for the telecommunication services and bandwidth has boomed. To handle this ever increasing demand, high speed EO modulators operating over $100 \mathrm{GHz}$ are required [1]. The currently used EO modulators based on $\mathrm{LiNbO}_{3}$ are limited in their bandwidth to $40 \mathrm{GHz}$, because at higher frequencies the velocity mismatch between the optical wave and the electrical traveling wave yields too large dephasing and consequently too short interaction lengths. To handle such high modulation frequencies a better performing material is required. Second order nonlinear optical (NLO) polymers [2,3] have been proposed to be useful candidates for this application already two decades ago. Though NLO polymers have the potential for high nonlinearity, the nonlinearity of the individual chromophores does not translate completely onto a macroscopic scale. This is because electrostatic intermolecular interactions lead to antiparallel clustering of the chromophores during electric field induced poling. These intermolecular interactions can be reduced by modifying the shape of the chromophore (making it more spherical) and thereby preventing their closer approach [4]. For long term applications the chromophores should have a high photochemical stability at the operating wavelength and a high stability of the poling order at the operating temperature. TCVDPA has the highest reported photochemical stability [5]. In addition, its NLO figure of merit is higher than dimethylaminonitrostilbene (DANS) and Disperse Red (DR1). The difference between the glass transition temperature $\left(\mathrm{T}_{\mathrm{g}}\right)$ of the polymer and the operating temperature, which determines the stability of the poling order, requires polymers with a high $\mathrm{T}_{\mathrm{g}}$ of about 200 ${ }^{\circ} \mathrm{C}$. When the NLO chromophores are incorporated in a photodefinable polymer, electrooptically active waveguides can be obtained by direct photodefinition. Compared with the reactive ion etching technique, this requires less processing steps and yields less sidewall roughness. This letter reports for the first time the direct photodefinition of an electrooptic polymer, i.e. TCVDPA in the SU8 host. Improvement of the poling efficiency by modifying TCVDPA with bulky side groups will also be presented.

Materials: The TCVDPA chromophore (Fig. 1a, $\mathrm{R}=\mathrm{H}$ ) was used in the current work. It consists of a single benzene electron bridge between a tricyanovinyl acceptor and a diarylamino donor. For photodefinition, TCVDPA is incorporated as a guest in the SU8 
(Fig. 1b) host. TCVDPA with different bulky groups $(\mathrm{R}=$ tert-butyl, methoxy $(\mathrm{MeO})$ and tert-butyl-dimethylsilane (TBDMS)) were prepared to study the inhibition of the intermolecular interactions. The $\mathrm{T}_{\mathrm{g}}$ of uncrosslinked SU8 is $60^{\circ} \mathrm{C}$. By crosslinking, the $\mathrm{T}_{\mathrm{g}}$ can be raised to about $200{ }^{\circ} \mathrm{C}$.

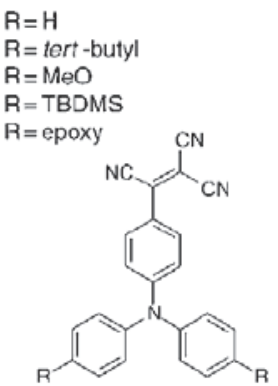

a

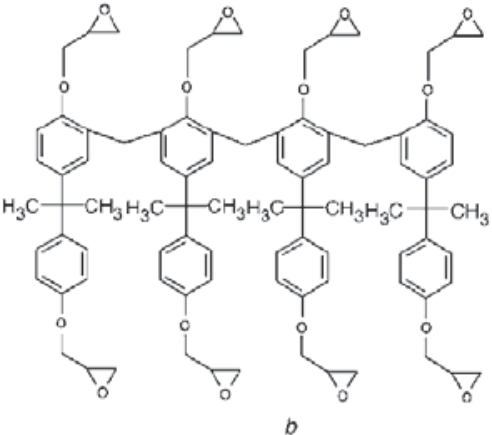

b

Fig. 1 TCVDPA and its modifications; $a$ : TCVDPA and its modifications, $b$ : SU8

Phodefinition: Photodefinition of the SU8-TCVDPA guest-host (GH) polymer is demonstrated by exploiting the low UV absorption window of the TCVDPA chromophore shown in Fig. 2, in which region most of the other NLO chromophores are highly absorbing. The photodefinition process is shown in Fig. 3. SU8 $5 \mathrm{wt} \%$ TCVDPA was spun on a Si substrate at $3000 \mathrm{rpm}$ to give a film thickness of $2 \mu \mathrm{m}$. It was then baked at $95^{\circ} \mathrm{C}$ for 5 minutes on a hot plate (soft bake). Subsequently, the polymer film was exposed to UV light with a $\mathrm{Cr}$ contact mask for 5 minutes in a Karl Süss mask aligner MA55 (365 nm, 405 $\mathrm{nm}$ and $435 \mathrm{~nm}$ ) and cured at $95^{\circ} \mathrm{C}$ for 5 minutes (post bake). The unexposed regions are uncrosslinked and are removed by development.

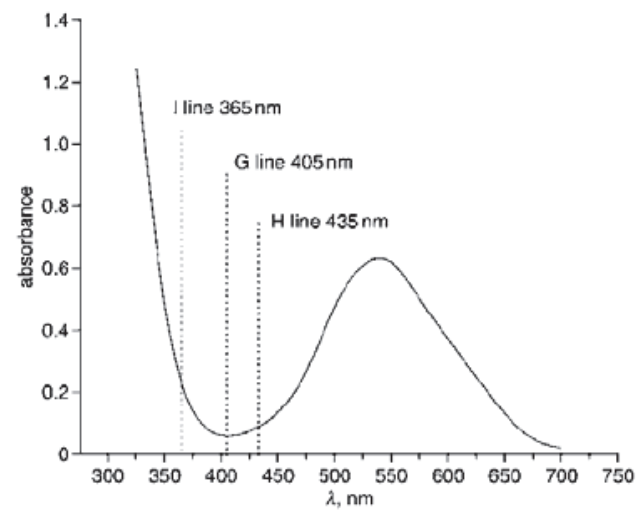

Fig. 2 Absorption spectrum of TCVDPA 


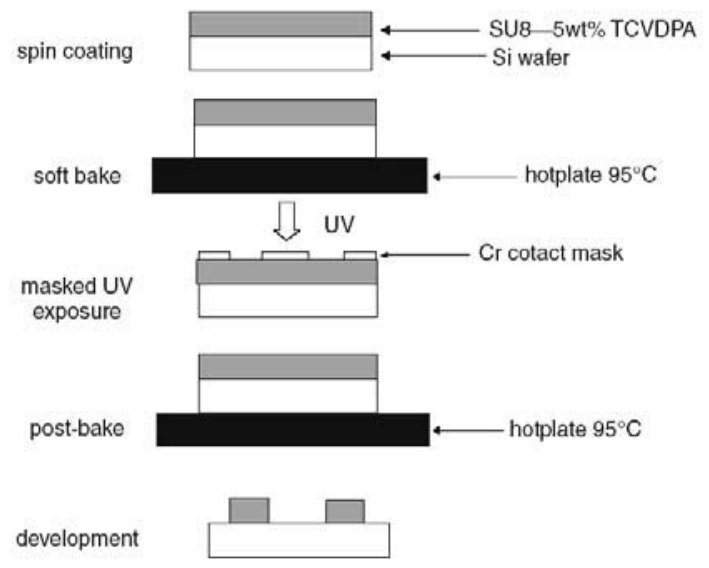

Fig. 3 Photodefinition process

Poling: The polymer should be noncentrosymmetric in order to exhibit second order nonlinearity. This is done by poling whereby the polymer is heated to its glass transition temperature $\left(\mathrm{T}_{\mathrm{g}}\right)$ and the chromophores are aligned by applying an electric field typically in the order of $100 \mathrm{~V} / \mu \mathrm{m}$. Poling was carried out in a crosslinked polymer film and not in a photodefined waveguide in order to have large poled region for measurement of the $\mathrm{r}_{33}$. A $2 \mu \mathrm{m}$ thick SU8 film with $25 \mathrm{wt} \%$ TCVDPA was spun on a glass wafer coated with an ITO (Indium Tin Oxide) layer which serves as the bottom electrode for poling. The film was then heated on a hot plate at $95^{\circ} \mathrm{C}$ for 1 hour and in the oven at $95^{\circ} \mathrm{C}$ for 8 hours under vacuum to remove the solvent. The film was exposed to UV light in a Karl Süss Mask aligner MA55 for 2 minutes and cured at $95^{\circ} \mathrm{C}$ for 15 minutes. A $100 \mathrm{~nm}$ gold layer was sputtered on the polymer as the top electrode for poling. It was observed that when equipped with TCVDPA as guest, the $\left(\mathrm{T}_{\mathrm{g}}\right)$ of the crosslinked SU8 is reduced considerably due to the plasticizing effect of the chromophores. The $\mathrm{T}_{\mathrm{g}}$ of the cured sample was around $45^{\circ} \mathrm{C}$. Therefore the sample was poled by heating it to $45^{\circ} \mathrm{C}$ and applying an electric field of $100 \mathrm{~V} / \mu \mathrm{m}$. Poling was done for about 40 minutes. The sample was cooled to room temperature and the electric field was removed. An $r_{33}$ coefficient of $6 \mathrm{pm} / \mathrm{V}$ was measured at $830 \mathrm{~nm}$ immediately after poling by the Teng-Man reflection technique [6]. In order to make a comparative study, the $\mathrm{r}_{33}$ coefficient was also measured in a high $\mathrm{T}_{\mathrm{g}}$ polymer, polysulfone (PS host) at different wt $\%$ of TCVDPA (guest). All the $\mathrm{r}_{33}$ values were measured at $830 \mathrm{~nm}$. This was poled at its $\mathrm{T}_{\mathrm{g}}$ under similar poling conditions as SU8 - $25 \mathrm{wt} \%$ TCVDPA. The $\mathrm{T}_{\mathrm{g}}$ of polysulfone as a function of the $\mathrm{wt} \%$ of TCVDPA is shown in Fig. 4. Fig. 4 also shows the $r_{33}$ of the PS-TCVDPA measured at different concentrations of TCVDPA. A deviation from the linear behavior was observed above $15 \mathrm{wt} \%$ of TCVDPA. The measured $r_{33}$ was $9 \mathrm{pm} / \mathrm{V}$ at $25 \mathrm{wt} \%$ TCVDPA. The difference in $\mathrm{r}_{33}$ coefficients between SU8 - $25 \mathrm{wt} \%$ TCVDPA $(6 \mathrm{pm} / \mathrm{V})$ and PS - $25 \mathrm{wt} \%$ TCVDPA (9 
$\mathrm{pm} / \mathrm{V}$ ) can be attributed to the fact that the $\mathrm{T}_{\mathrm{g}}$ of the cured SU8 - $25 \mathrm{wt} \%$ TCVDPA is so close to room temperature (about $45^{\circ} \mathrm{C}$ ) that the poling order relaxes rapidly when the poling field is removed at room temperature. This is can be prevented by equipping TCVDPA with epoxy groups (Fig. 1a, $\mathrm{R}=$ epoxy) and crosslinking TCVDPA-epoxy with SU8.

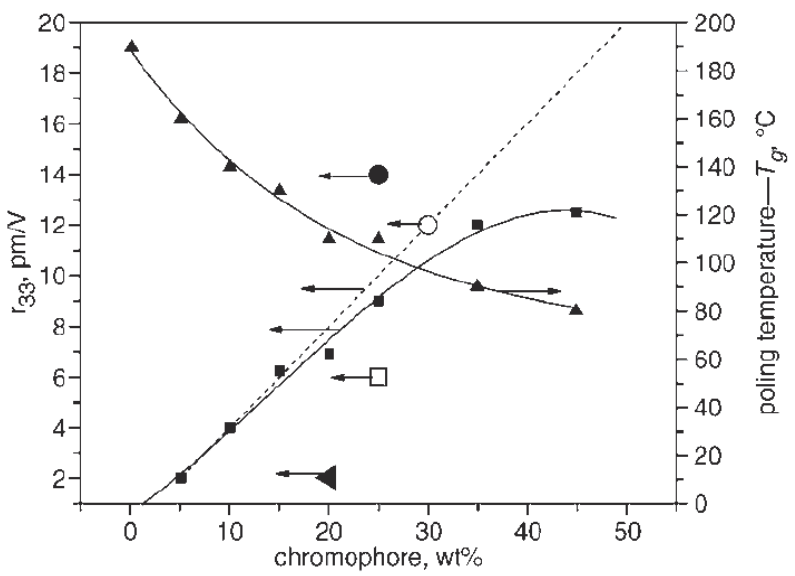

Fig. 4 Measured r33 coefficients for different TCVDPA modifications in SU8 and PS at $830 \mathrm{~nm}$
r33 PS - TCVDPA
$\triangle$ Tg PS - TCVDPA
- - - r33 PS - TCVDPA linear fit
r33 SU8 - TCVDPA
r33 PS - TCVDPA - tert-butyl
r33 PS - TCVDPA-MeO
r33 PS - TCVDPA - TBDMS

The poling behavior of TCVDPA-tert-butyl, TCVDPA-MeO, and TCVDPA-TBDMS was studied by incorporating them as guests in PS at concentrations at which the $\mathrm{r}_{33}$ of PS TCVDPA deviates from linear behavior. The measured $r_{33}$ values are shown in Fig. 4. At $25 \mathrm{wt} \%$, TCVDPA tert-butyl shows an enhancement in $\mathrm{r}_{33}$ of $5 \mathrm{pm} / \mathrm{V}$, and at $30 \mathrm{wt} \%$ TCVDPA-MeO shows an enhancement of $2 \mathrm{pm} / \mathrm{V}$ compared with TCVDPA. These data clearly demonstrate the effect of the bulky groups. In the case of TCVDPA-tert-butyl the enhancement in $r_{33}$ is even higher than that predicted by the linear fit. This indicates that some intermolecular interactions are already present even at low TCVDPA concentration $(<15 \mathrm{wt} \%)$ in PS. In contrary to our expectations, at $20 \mathrm{wt} \%$ TCVDPA-TBDMS gave an $\mathrm{r}_{33}$ of only $2 \mathrm{pm} / \mathrm{V}$ compared with $7 \mathrm{pm} / \mathrm{V}$ measured with TCVDPA. This can be explained by the high molecular weight of TCVDPA-TBDMS which not only reduces the chromophore number density for a given $w t \%$, but also reduces the poling efficiency due to the restricted motion of the chromophores. 
Conclusions: For the first time photodefinition of an electro-optic polymer has been demonstrated with the SU8 - TCVDPA system using the low UV absorption window of TCVDPA chromophore. The crosslinked polymer system was poled and an $\mathrm{r}_{33}$ of $6 \mathrm{pm} / \mathrm{V}$ at $830 \mathrm{~nm}$ was measured. Increasing the $\mathrm{r}_{33}$ by the attachment of bulky groups to the chromophore to inhibit intermolecular interactions was demonstrated.

This research work is supported by the Dutch Technology Foundation STW, Applied Science Division of NWO and the Technology Program of the Ministry of Economic Affairs and carried out within the framework of the project TOE 6067 "Broadband and wavelength selective modulators for optical communication based on electro-optic polymers"

IEE 2006

Electronics Letters online no: 20063535

doi: 10.1049/el:20063535

M. Balakrishnan, M.B.J. Diemeer and A. Driessen (Integrated Optical

Microsystems, University of Twente, MESA+ Institute for Nanotechnology,

P.O. Box 217, 7500 AE Enschede, The Netherlands)

E-mail: m.balakrishnan@ewi.utwente.nl

M. Faccini, W. Verboom and D.N. Reinhoudt (Laboratory of Supramolecular

Chemistry and Technology, University of Twente, MESA+ Institute for Nanotechnology, P.O. Box 217, 7500 AE Enschede, The Netherlands)

A. Leinse (LioniX BV, P.O. Box 456, Enschede 7500 AH, The Netherlands)

\section{References:}

[1] Y. Shi, C. Zhang, H. Zhang, J. Betchel, L. R. Dalton, B. Robinson, W. Steier, "Low half voltage polymeric electro-optic modulator achieved by controlling chromophore shape", Science, 288, p119, 2000.

[2] G. R. Meredith, J. G. Van Dusen, D. J. Willams : “Optical and nonlinear optical characterization of molecularly doped thermotropic liquid crystalline polymers", Macromolecules, 15, p1385, 1998.

[3] D. Cheng, H. R. Fetterman, A. Chen, W. H. Steier, W. Wang, Y. Shi : "Demonstration of $110 \mathrm{GHz}$ electro-optic polymer modulators", Appl. Phys. Lett., 70, p3335, 1997.

[4] B. H. Robinson, L. R. Dalton : "Monte carlo statistical mechanical simulations of the competition of intermolecular electrostatic and poling field interactions", $J$. Phys. Chem. A, 104, p4785, 2000.

[5] A. G. Gonzales, G. I. Stegeman, A. K. Y. Jen, X. Wu, M. Canva, A. C. Kowalkzyk, X. Q. Zhang, H. Z. Lackritz: "Photostability of electro-optic polymers possessing chromophores with efficient amino donors and cyano containing acceptors", J. Opt. Soc. Am. B, 18, p1846, 2001.

[6] C. C. Teng, H. T. Man : "Simple reflection technique for the measuring the electrooptic coefficient of poled polymers", Appl. Phys. Lett., 56, p1734, 1990. 
Electronics Letters Paper 


\section{List of Publications}

M. Balakrishnan, M. Faccini, M. B. J. Diemeer, E. J. Klein, G. Sengo, A. Driessen, W. verboom, D. N. Reinhoudt, "Microring resonator based modulator made by direct photodefinition of an electro-optic polymer". Applied physics letters, 92 (15). pp 153310-1 to 153310-3. ISSN 0003-6951, 2008.

M. Faccini and M. Balakrishnan and M.B. J. Diemeer, Z. Hu, K. Clays, I. Asselberghs, A. Leinse A. Driessen D. N. Reinhoudt W. Verboom "Enhanced poling efficiency in highly thermal and photostable nonlinear optical chromophores", Journal of materials chemistry, 18. pp 2141-2149. ISSN 0959-9428, 2008.

M. Faccini, M. Balakrishnan, M. B. J. Diemeer, R. Torosantucci, A. Driessen, D. N. Reinhoudt, W. Verboom "Photostable NLO Polycarbonates", Journal of materials chemistry, DOI: 10.1039/<B810274K> (Accepted for publication 2008).

M. Balakrishnan, M. Faccini, M. B. J. Diemeer, W. Verboom, A. Driessen, D. N. Reinhoudt, A. Borreman and M. J. Gilde, "Photodefinable electro-optic polymer for high speed modulators", Electronics Letts, 42 (1), pp 51-52, 2006.

M. Balakrishnan, M. Faccini, M. B. J. Diemeer, W. Verboom, A. Driessen, D. N. Reinhoudt, A. Borreman and M. J. Gilde, "Highly stable and low loss electro-optic polymer waveguides for high speed modulators using photodefinition technique", Photonics West, SPIE, vol 6123, p 61230J, 2006.

M. Balakrishnan, E. J. Klein, M. Faccini, M. B. J. Diemeer, W. Verboom, A. Driessen, D. N. Reinhoudt, A. Leinse, "Fabrication of an electro-optic polymer microring resonator", Proceedings IEEE/LEOS Benelux, pp 73-76, 2006.

M. Faccini. M. Balakrishnan M. B. J. Diemeer, A. Leinse W. Verboom, A. Driessen, D. N. Reinhoudt, "Photodefinable electro-optic polymers for high speed modulators", International Conference on Organic Photonics and Electronics - ICOPE 2006, pp 71-72, 2006.

M. Balakrishnan, M. Faccini, M. B. J. Diemeer, W. Verboom, A. Driessen, D. N. Reinhoudt, A. Borreman and M. J. Gilde, "Electro-optic polymers for high speed modulators", Proceedings IEEE/LEOS Benelux, pp 313-316, 2005.

M. B. J. Diemeer, R. Dekker L. Hilderink, A. Leinse, M. Balakrishnan, M. Faccini, A. Driessen "Polymeric components for telecom and datacom", SPIE 5956, ISSN 0277- 786X ISBN 0-8194-5963-1, 2005.

M. Faccini, M. Balakrishnan, M. B. J. Diemeer, A. Leinse, W. Verboom A. Driessen D. N. Reinhoudt, "Photodefinable electro-optic polymers for high speed modulators". ICONO 8, Japan, 2005. 
M. Balakrishnan, M. Faccini, M. B. J. Diemeer, W. Verboom, A. Driessen, D. N. Reinhoudt, A. Borreman and M. J. Gilde, "Photodefinition of channel waveguides in Electro-Optic polymer", Proceedings IEEE/LEOS Benelux, pp 76-80, 2004. 


\section{Acknowledgments}

I am thankful to Alfred Driessen and Mart Diemeer for giving me the opportunity to work in IMOS group. I am thankful to Alfred Driessen for his useful discussions and ideas during the past four years and for spending a lot of time in patiently correcting my thesis outside his normal working schedule. I am thankful to Mart Diemeer for his close guidance and all instantaneous ideas and for steering the work in the right direction and also for all his excellent feedback which gave the exact status of the progress. Mart Diemeer is no doubt the knowledge warehouse about polymers and an one-stop shop for all polymer related queries. I would like to thank Mirko Faccini for his hard efforts to make mgs of polymers and for all fruitful discussions and nice co-ordinnation. I would like to thank Wim Verboom for the collaboration between SMCT and IOMS. I would like to express my thanks to all the committee members for spending time in reading my thesis and for their valuable comments. I am very much thankful to Anton Hollink for all innumerable help and assistance and for his extreme patience. Without Anton it would definitely be difficult for PhDs to work independently. I am greatly indebted to Rita ter Weele for all the more than needed help provided in official and also personal things. I would to thank all IOMS staff members - Markus Pollnau, Hugo Hoekstra, Rene de Ridder, Kerstin Woerhoff, for their thought provoking questions and discussions during the group talks. I am very much thankful to Arne Leinse for his dynamic participation in the project although he joined us only in the middle and had to leave before the end. I am thankful to Feridun Ay for helping me with prism coupling measurements and for his friendly chats at our usual meeting place in front of the building. I would like to thank Edwin Klein for useful discussions about microrings. I would like to thank Ronald Dekker for introducing me to PM500 setup. I would like to thank Gabriel Sengo for all the final work which we did together. I would like to thank Lucie Hilderink for making me familiar with clearroom equipments and for patiently cleaning the LDG spinner when I sometimes forget to clean it. I would like to thank Marcel Hoekman and Nur Ismail for their help in curve loss fitting. I would like to thank Marcel Hoekman and Chaitanya Dongre for all their help and support during Electrodynamics classes. I would to thank Amol and Jonathan Bradley for their company in the gym. I would like to thank my office room mates Jing Yang, Lasse Kauppinen, Henry Uranus for providing a cordial working atmosphere. I would to thank Lasse Kauppinen for accompanying me to the mensa every day. I would like to thank Shanmugam Aravazhi for his encouraging words and providing many practical and useful informations regarding many things. I would like to thank Dimitri Geskus for sepending time in translating Dutch letters. I would to thank all my friends in the UT namely Raagav, Anand, Vishnu, Shankar, Bijoy, Balaji, Tamalika ...... for their enjoyable company during the past four years. I would like to thank Bremkumar for all his humorous talks over phone and for his support and advise during the past years. I would like to thank my parents for their constant love and care which they shower upon me and their persistent concern over my studies. I am thankful to Sarasvathi aunty for all the inspiration provided right from my childhood. Last but not the least I would like to thank my wife for all the support she has provided in the past months especially while writing my thesis. 